Noah Benjamin Wallace

77 Words

\title{
A Precision Measurement of the Magnetic Moment of the Omega Minus Hyperon
}

The magnetic moment of the $\Omega^{-}$hyperon has been measured to high precision. Using the spin precession technique on a sample of $2.4 \times 10^{5}$ polarized $\Omega^{-}$hyperons produced by both polarized and unpolarized neutral beams the magnetic moment of the $\Omega^{-}$hyperon was measured to be $2.024 \pm 0.056$ nuclear magnetons. This is the highest precision measurement of the magnetic moment of the $\Omega^{-}$to date. The results are in good agreement with the value measured by a previous experiment. 


\title{
UNIVERSITY OF MINNESOTA
}

This is to certify that I have examined this bound copy of a doctoral thesis by

\author{
Noah Benjamin Wallace
}

and have found that it is complete and satisfactory in all respects and that any and all revisions required by the final examining committee have been made.

Professor Kenneth J. Heller

(Faculty Adviser)

GRADUATE SCHOOL 


\title{
A Precision Measurement of the Magnetic Moment of the Omega Minus Hyperon
}

\author{
A THESIS \\ SUBMITTED TO THE FACULTY OF THE GRADUATE SCHOOL \\ OF THE UNIVERSITY OF MINNESOTA \\ BY
}

Noah Benjamin Wallace

IN PARTIAL FULFILLMENT OF THE REQUIREMENTS

FOR THE DEGREE OF

DOCTOR OF PHILOSOPHY

May, 1995 
(C) Noah Benjamin Wallace 1995

ALL RIGHTS RESERVED 


\title{
A Precision Measurement of the Magnetic Moment of the Omega Minus Hyperon
}

\author{
by Noah Benjamin Wallace \\ Under the supervision of Professor Kenneth J. Heller
}

\begin{abstract}
The magnetic moment of the $\Omega^{-}$hyperon has been measured to high precision. Using the spin precession technique on a sample of $2.4 \times 10^{5}$ polarized $\Omega^{-}$hyperons produced by both polarized and unpolarized neutral beams the magnetic moment of the $\Omega^{-}$hyperon was measured to be $2.024 \pm 0.056$ nuclear magnetons. This is the highest precision measurement of the magnetic moment of the $\Omega^{-}$to date. The results are in good agreement with the value measured by a previous experiment.
\end{abstract}




\section{Acknowledgements}

Running and analyzing a high energy physics experiment is a time consuming and trying business. I was lucky enough to have a huge number of people who kept me operating at something close to tolerences while I was involved with this experiment.

Some people are lucky enough to run into the person who gives their life the meaning it was intended to have. I was fortunate enough to meet the love of my life while working at fermilab. This thesis is dedicated to Michela, who gave me a new understanding of the way life could be. Thanks to fermilab housing who put the two of us in the same dormitory.

My family worked very hard to keep me away from the edge of the chasm. One doesn't simply thank one's family but maybe that is all I can do here. So to my Mother Linda, Father Neil, Brother Eli, Karen, Roger, and Connie, as well as, my Grandparents Leonard and Mary Feist, Rose and postumously Sam Wallace, and my Aunt Betsy Feist, thank you very much for the support only a family can give.

The E800 group was like a large and slightly disfunctional family running a chaotic 24 hour mom and pop store. The memory of the pressure we endured together has faded over time but we had and still have some really great experiences that we all recall during reunions. I would like to thank my advisor Ken Heller for his support 
and knowledge on many subjects physical and political. Gina Rameika held the role as adult to an experiment full of children.

This experiment ran on the sweaty backs of a few people. In particular Pete Border who taught me all the experimental techinque I'll ever need to know. Dave Ciampa who made me a stronger individual both physically and emotionally.

Once in a while you meet somebody in this field who doesn't need to threaten you to make you do what seems impossible. For me Ken Johns is that person. Ken could lead the graduate students thorugh a mine field all the while making sure none of them got so much as a ingrown toe nail. I have nothing but respect for Ken. He has been and is an example I will never be able to follow.

The other graduate students on this experiment bore the brunt. Jerry Guglielmo and Dave Woods carried my sorry butt all the way through and I can't say enough for their ability as physicists and people. They really made sure this thing happened the way it was supposed to happen.

On an experiment like this there are always a number of part timers who help more than they think they do.

Eileen Berman who is more than our computing liason she's a member of our family.

Graham Allan, Eric James, Dave Fein, Alex Ayala-Mercado, Jamal Jallilian-Marian, Aaron Larson, Milena Grobelewski-Higgins, Patricia Tynan, Vic DiCarlo, and Randy McGriff who came up big when it was really necessary. 
I would also like to thank huge number of people at Fermilab who helped with either technical support or just support during the two an a half years I lived at the lab.

Lou Morris, and Jean Wilson our most excellent technicians and good friends too, Ron Miksa at special stores, Stephen Pordes for all his support, Gordon Gillespie and Karen Kephart at Lab 6.

There are some people at Fermilab who need special thanks:

Judy Thomas with love for serving me and teaching me to make the finest Margaritas on the planet, as well as, first class bartending of all sorts and a good word always.

The Arizona guys: Andy Milder, Dave Fein, and Eric "E.J." James for late night hoops, heavy beer consumption, verbal abuse, and fabulous golf. Some of the best companions one could hope for.

A special thanks to Mykha of Mykha's Place the "Kitchen Goddess".

The gym rats of Fermilab: Ryan "Small World" Haggler, Carl Penson, and the rest who gave me remedial hoops and "smack" talking lessons that wil stay with me always, and a nickname that I hope I can shake someday.

Here at home I am really lucky to have a huge number of friends and associates who help me maintain a semblance of an existance.

Lori Greene for being my closest friend and confidant for 15 years. Without you I don't know where I would be. 
Joel Shapira for knowing why "The Bad Leutenent" is one of the best films of all time, and the other members of the "Doug Little Four" for sharing part of your great thing with me. You guys are some Bad-Axe Motherfletchers.

John and Salina and, Graham and Genie, for putting up with me on so many occasions even when I was the most horrible company in the world. You guys kept me in there.

Local hoops: John Neely, Aaron Larson, Josh Larson, Johnathan Hausman, Phil Kraus, Mark Granowski, "Small" Paul Granowski, and the guys from SAP. Dave, Dave, Dave, Paul, Chris, Matt, John and John and all the rest from the Rec Center. Doug, Mike, Dave, Donnie and the rest of the guys from the St. Paul Gym.

People who make things work for me: Tim Foley, Steve "Weef" Shapira, Miodrag "Misha" Drashcovich, and Robbie Riech at Midway Motel. John Cappriotti, Sue Kasahara, Dave DeMuth, Dave Dikeman, Phil Kraus, Denise Leezer, and Liz Dailey for putting up with my ranting both at and away from work.

Last I would like to thank my brother Eli one more time. He is my example and my competitor, he goes where I dare not, and does things I cannot, both on the court and in his life. Thanks bro, you might still know how to play the game. 


\section{Dedication}

This thesis is dedicated to Michela.

Tu sei la passione della mia vita. 


\section{Table of Contents}

Abstract

Acknowledgements

Dedication vi

List of Tables $\quad \mathrm{x}$

List of Figures $\quad$ xiv

List of Figures

List of Tables

1 Introduction 1

1.1 Magnetic Moments and Baryon Structure . . . . . . . . . . . 1

1.1.1 Symmetry Based Models . . . . . . . . . . . . . . . . 2

1.1.2 QCD based models ................. 5

1.2 The Omega Minus Magnetic Moment . . . . . . . . . . . . . . 8

1.3 Magnetic Moments by the Spin Precession Technique . . . . . . . . . . . 10

2 The Apparatus 14

2.1 The Primary Beam . . . . . . . . . . . . . . . . . 14 
2.2 Secondary and Tertiary Beams . . . . . . . . . . . . . . . 15

2.2 .1 Targeting Dipoles . . . . . . . . . . . . . . . . 16

2.2 .2 The Targets . . . . . . . . . . . . . . . 16

2.2 .3 The Neutral Collimator . . . . . . . . . . . . . . . 17

2.2.4 The Precession Magnet and Charged Collimator . . . . . . . 19

2.3 Operating Modes . . . . . . . . . . . . . . . . . . . . 19

2.4 The Charged Particle Spectrometer . . . . . . . . . . . . . . . . 22

2.4.1 General Design of the Spectrometer . . . . . . . . . . . . 22

2.4.2 The Scintillation Counters . . . . . . . . . . . . . . . . 24

2.4.3 The Silicon Strip Detectors (SSDs) . . . . . . . . . . . 26

2.4.4 The Multi-wire Proportional Chambers (MWPCs) . . . . . . 27

2.4.5 The Analysis Magnets . . . . . . . . . . . . . . . . . . . . 29

2.5 The Trigger . . . . . . . . . . . . . . . . . . 31

2.6 The Data Aquisition System . . . . . . . . . . . . . . . . . 32

2.6.1 Operational Overview . . . . . . . . . . . . . . 32

2.6 .2 Software . . . . . . . . . . . . . . . 33

2.6.3 Hardware . . . . . . . . . . . . . . . . . . 34

3 Event Reconstruction and Data Selection 36

3.1 Introduction . . . . . . . . . . . . . . . . 36

3.2 The First Pass . . . . . . . . . . . . . . . . . 37

3.3 Full Reconstruction . . . . . . . . . . . . . . . . . 38

3.4 The Monte Carlo Simulation . . . . . . . . . . . . . . . . . . . 41

3.5 Background and Data Selection Criteria . . . . . . . . . . . . . 44

3.6 Reconstructed Samples and Particle Yields . . . . . . . . . . . . 53

4 Analysis $\quad 56$ 
4.1 Magnetic Moment Analysis . . . . . . . . . . . . . . . . . 56

4.2 Omega Minus and Lambda Polarizations and Angular Distributions . . 57

4.2 .1 Lambda Polarization . . . . . . . . . . . . . . . . . 62

4.3 The Acceptance Cancellation Method . . . . . . . . . . . . 62

4.4 The Hybrid Monte Carlo Technique . . . . . . . . . . . . . . . . . 64

4.4.1 Analysis Selection Criteria . . . . . . . . . . . . . 65

4.4 .2 Biases and Polarization . . . . . . . . . . . 66

4.5 Performance of the Polarization Analysis . . . . . . . . . . . . . . 68

4.6 The Master Fit . . . . . . . . . . . . . . . . . . . . . . 68

5 Results and Systematic Uncertainty Studies $\quad 71$

5.1 Omega Minus Polarization Results . . . . . . . . . . . . . 71

5.2 Omega Minus Magnetic Moment Results . . . . . . . . . . . . . . 73

5.2.1 Higher Order Precession Angles . . . . . . . . . . . . 73

5.3 Systematic Studies . . . . . . . . . . . . . . . . 76

5.3.1 The Effect of the Selection Criteria . . . . . . . . . . . 79

5.3 .2 Sample Contamination . . . . . . . . . . . . 82

5.4 Internal Checks . . . . . . . . . . . . . . . . . . . . 82

5.4 Consistancy Checks .................. 82

5.4 .2 Acceptance Cancellation Results ............. 85

5.4 .3 Cascade Minus Results . . . . . . . . . . . . . . . . 87

6 Implications $\quad 92$

$\begin{array}{ll}\text { References } & 95\end{array}$ 


\section{List of Tables}

1.1 The baryon spin-flavor $\mathrm{SU}(6)$ wavefunctions. . . . . . . . . . 3

1.2 Static quark model predictions. The experimental results are also listed for comparison. ..................... 3

1.3 Magnetic moment predictions for various sum rule calculations. . . . . 5

1.4 Magnetic moment predictions for various bag model calculations. . . . . 7

1.5 Magnetic moment predictions for lattice gange calculations. . . . . . . 8

1.6 Theoretical predictions for the $\Omega^{-}$magnetic moment. The measured value is included for comparison . . . . . . . . . . . . . . 9

2.1 The $\int B d l$ and central orbit momentum for the 2 currents used in the sweeping magnet. . . . . . . . . . . . . . . . . . . 19

2.2 The z-positions and size of the detectors in the E800 spectrometer. . . . 26

2.3 The voltages and efficiencies of the detectors. . . . . . . . . . . 28

2.4 The singles rates for various detectors and triggers for three production modes at typical running intensities. . . . . . . . . . . . . 32

2.5 The number of runs and three-track triggers taken under various run conditions. . . . . . . . . . . . . . . . . . 34

3.1 The first pass . . . . . . . . . . . . . . 37

3.2 The first pass analysis event totals. . . . . . . . . . . . . . 38 
3.3 The percentage of .ds0 events reconstructed by various reconstruction routines. An event was reconstructed correctly when there were two or more correctly assigned hit for each track both upstream and downstream of the analysis magnet in the $\mathrm{x}$-view and three or more correctly assigned hits for each track in the y-view. . . . . . . . . . . . . . . . 42

3.4 The ratio of monte-carlo events for various backgrounds compared to $\Omega^{-} \rightarrow \Lambda \mathrm{K}^{-}$events accepted and surviving data selection criteria. . . . 49

3.5 The total $\Omega^{-}$and $\Xi^{-}$samples and the approximate yields per incident proton. .................... 5

4.1 The input and output polarizations, biases, and $\chi^{2}$ s for unreconstructed $\Omega^{-}$monte-carlo samples. The $\chi^{2}$ 's are based on 19 degrees of freedom for the $20 \cos \theta_{p}$ bins in each view. . . . . . . . . . . . 69

4.2 The input and output polarizations, biases, and $\chi^{2}$ 's for reconstructed $\Omega^{-}$monte-carlo samples. The $\chi^{2}$ 's are based on 19 degrees of freedom for the $20 \cos \theta_{p}$ bins in each view. . . . . . . . . . . . 69

5.1 The data sets making up the final $\Omega^{-}$data sample. . . . . . . . . . . . 71

5.2 The measured $\Omega^{-}$polarizations, biases, and $\chi^{2}$ 's for the neutral production sample with $\int \mathrm{Bdl}=24.36 \pm 0.24 \mathrm{~T} \mathrm{~m}$. The $\chi^{2}$ s are based on 20 degrees of freedom for the $20 \cos \theta_{p}$ bins in each view. . . . . . 72

5.3 The momentum averaged measured $\Omega^{-}$polarizations, biases, and $\chi^{2}$ s for the three production methods. The $\chi^{2}$ 's are based on 20 degrees of freedom for the $20 \cos \theta_{p}$ bins in each view. . . . . . . . . 72

5.4 The field integrals $\int \mathrm{Bd}$ and precession angles $(\Phi)$ for Fermilab E800 and Fermilab E756 $\Omega^{-}$magnetic moment measurements. . . . . . . . . . 76

5.5 The $\chi^{2}$ per degree of freedom for the linear $\Phi$ vs $\int B d l$ using E800 data only and using E756 and E800 data. . . . . . . . . . . 76 
5.6 The precession angles $(\Phi)$ and $\Omega^{-}$magnetic moment results for the neutral production sample at $\int \mathrm{Bdl}=24.36 \mathrm{~T} \cdot \mathrm{m} . \ldots \ldots . \ldots 78$

5.7 The momentum averaged measured precession angles $(\Phi)$ and $\Omega^{-}$magnetic moment results for the three production methods. . . . . . . . 78

5.8 The measured $\Omega^{-}$polarizations, precession angles, and magnetic moment for four momentum bins of neutral production sample with $\int \mathrm{Bdl}=24.36$ $\mathrm{T} \cdot \mathrm{m}$ and momentum averaged results for the four data data samples. This analysis used the tight mass cuts at $\pm 7.5 \mathrm{MeV} \ldots \ldots . \ldots 80$

5.9 The measured $\Omega^{-}$polarizations, precession angles, and magnetic moment for four momentum bins of neutral production sample with $\int \mathrm{Bdl}=24.36$ $\mathrm{T} \cdot \mathrm{m}$ and momentum averaged results for the four data data samples. This analysis used the tight $\chi_{G}^{2} /$ d.f. cuts at $3.0 /$ d.f. and $\chi_{K}^{2} 10.0 \ldots 81$

5.10 The master fit results for the analysis using three selection criteria. . . . 81

5.11 The measured $\Omega^{-}$polarizations, precession angles, and magnetic moment for four momentum bins of neutral production sample with $\int \mathrm{Bdl}=24.36$ $\mathrm{T} \cdot \mathrm{m}$ and momentum averaged results for the four data data samples. These samples have $2.5 \% \Xi^{-}$events included in the sample. . . . . . . .

5.12 The input and analyzed polarizations for monte carlo $\Omega^{-}$neutral production events with increasing levels of $\Omega^{-} \rightarrow \Xi^{0} \pi^{-}$pollution. . . . . . 84

5.13 The measured $\Omega^{-}$polarizations, precession angle and magnetic moment for the neutral production sample with $\int \mathrm{Bdl}=24.36 \pm 0.24 \mathrm{~T} \cdot \mathrm{m}$ using the acceptance cancellation method. . . . . . . . . . . . 86

5.14 The momentum averaged measured $\Omega^{-}$polarizations, precession angles, and magnetic moments for the four data samples analyzed using the acceptance cancellation method. . . . . . . . . . . 86

5.15 The measured $\Xi^{-}$polarizations the neutral production sample with and without the kinematic angle criteria $\int \mathrm{Bdl}=-24.36 \pm 0.24 \mathrm{~T} \mathrm{~m} . \ldots$ 
5.16 The measured $\Xi^{-}$polarizations the spin transfer production sample with and without the kinematic angle criteria $\int \mathrm{Bdl}=-24.36 \pm 0.24 \mathrm{~T} \cdot \mathrm{m} . \quad \ldots 90$

5.17 The momentum averaged measured $\Xi^{-}$polarizations and magnetic moments for four spin transfer $\Xi^{-}$samples at $\int \mathrm{Bdl}=-24.36 \ldots \ldots 90$

6.1 Theoretical predictions for the $\Omega^{-}$magnetic moment. The previously measured value is included for comparison. . . . . . . . . . . . . 92 


\section{List of Figures}

1.1 Producing polarized hyperons at a production angle. . . . . . . . . . 12

2.1 Schematic of the PC3 enclosure (elevation view) including magnets, targets (T1 and T2) and beam SWIC's (PC3WC2, PC3WC3, and PC3WC4 15

2.2 The E800 Neutral Collimator . . . . . . . . . . . . . . . . 18

2.3 The field integral of the precession magnet versus the magnet current. These measurements were made by fermilab experiment E756. . . . . . 20

2.4 The E800 Charged Collimator . . . . . . . . . . . . . . . . 21

2.5 Operating Modes . . . . . . . . . . . . . . . . . 23

2.6 The E800 Spectrometer (Plan View) . . . . . . . . . . . . 25

2.7 The plateau curve for chamber 6 . The vertical axis is the relative efficiency and the horizonal axis is the high voltage in $\mathrm{kV}$. The arrow indicates the operating voltage of the chamber. . . . . . . . . . 28

3.1 Schematic diagram of the E800 reconstruction program . . . . . . . 39

3.2 The geometric $\chi_{G}^{2}$ distribution for reconstructed data and monte carlo $\Omega^{-}$events. The monte carlo data are shown with the solid lines and the data with dots. The arrow denotes the reconstruction selection criteria.

3.3 The kinematic $\chi_{K}^{2}$ distribution for reconstructed data and monte carlo $\Omega^{-}$events. The monte carlo data are shown with the solid lines and the data with dots. The arrow denotes the reconstruction selection criteria. 
3.4 $\mathrm{X}_{T}$ (position in $\mathrm{x}$ in $\mathrm{cm}$. of the $\Omega^{-}$at the target) distribution for reconstructed data and monte carlo $\Omega^{-}$events. The monte carlo data are shown with the solid lines and the data with dots. The two arrows denote the reconstruction selection criteria. . . . . . . . . . . 45

3.5 $\mathrm{Y}_{T}$ (position in $\mathrm{y}$ in $\mathrm{cm}$. of the $\Omega^{-}$at the target) distribution for reconstructed data and monte carlo $\Omega^{-}$events. The monte carlo data are shown with the solid lines and the data with dots. The two arrows denote the reconstruction selection criteria. . . . . . . . . . . 46

3.6 The $\Omega^{-}$vertex distribution for reconstructed data and monte carlo $\Omega^{-}$ events. The monte carlo data are shown with the solid lines and the data

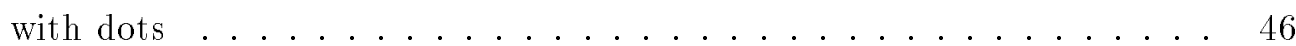

3.7 The $\Lambda$ vertex distribution for reconstructed data and monte carlo $\Omega^{-}$ events. The monte carlo data are shown with the solid lines and the

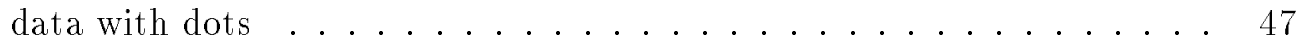

3.8 The total momentum distribution for reconstructed data and monte carlo $\Omega^{-}$events. The monte carlo data are shown with the solid lines and the data with dots. . . . . . . . . . . . . . . .

3.9 The $\Lambda$-K $\mathrm{K}^{-}$invariant mass distribution for reconstructed data and monte carlo $\Omega^{-}$events. The monte carlo data are shown with the solid lines and the data with dots. The two arrows denote the reconstruction selection

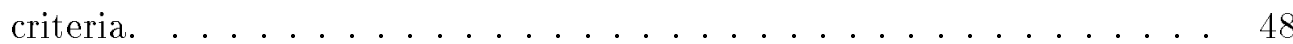

3.10 The $\Lambda-\pi^{-}$invariant mass distribution for reconstructed data and monte carlo $\Xi^{-}$events. The monte carlo data are shown with the solid lines and the data with dots. The two arrows denote the reconstruction selection criteria. ........................ 48

3.11 The $\cos \theta_{K}$ vs $\phi_{K}$ distribution for reconstructed monte-carlo $\Omega^{-}$events. $\quad 50$

3.12 The $\cos \theta_{K}$ vs $\phi_{K}$ distribution for reconstructed monte-carlo $\Xi^{-}$events. $\quad 50$ 
3.13 The $\cos \theta_{K}$ vs $\phi_{K}$ distribution for reconstructed data events. . . . . . . 51

3.14 The $\cos \theta_{K}$ vs $\phi_{K}$ distribution for reconstructed data events after the kinematic angle cut. . . . . . . . . . . . . . . 51

3.15 The $\Lambda-\pi^{-}$invariant mass distribution for reconstructed data events before and after the kinematic angle cut. . . . . . . . . . . 52

3.16 The $\Lambda$ - $\mathrm{K}^{-}$invariant mass distribution for reconstructed data events before and after the kinematic angle cut. . . . . . . . . . . .

3.17 The multiplicative factor determined from the bin by bin division of the data and monte carlo $\Xi^{-}$and $\Omega^{-}$mass distributions. The similarity in the shapes of the two plots indicates that the shape is in fact due to resolution. The difference in the size of the multipliers shows that there is indeed background in the $\Omega^{-}$sample and none in the $\Xi^{-}$sample. . . 54

3.18 The $\Lambda$-K $\mathrm{K}^{-}$invariant mass distribution for reconstructed $\Omega^{-}$events. The histogram is a resolution corrected monte carlo mass distribution. The dots are the mass distribution for the entire $\Omega^{-}$data sample used for

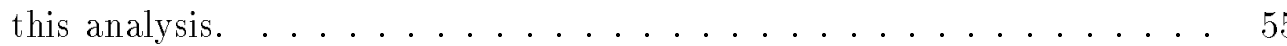

4.1 The relationship of the lab frame $(\hat{x}, \hat{y}, \hat{z})$ to the $\Lambda$ frame $(\hat{X}, \hat{Y}, \hat{\Lambda})$ showing the polar angles $\theta$ and $\phi \ldots \ldots \ldots \ldots$. . . . . . . . . 59

4.2 The proton direction in the $\Lambda$ coordinate system showing the polar angles

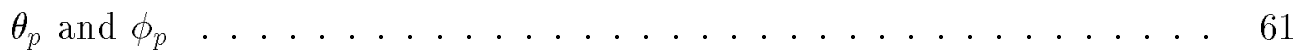

4.3 The proton distributions for x,y and $\mathrm{z}$ for a sample of $\Omega^{-}$events. . . . . 63

4.4 The difference between the generated and reconstructed $\cos \theta_{p}$ for x, y, z. 67

5.1 The polarization signals in $\mathrm{x}, \mathrm{y}, \mathrm{z}$ vs. momentum for the neutral production sample with $\int \mathrm{Bdl}=-24.36$ and the momentum averaged results for the four data samples analyzed using the hybrid monte carlo technique . 74 
5.2 The biases in $x, y, z$ vs. momentum for the neutral production sample with $\int \mathrm{Bdl}=-24.36$ and the momentum averaged results for the four data samples. . . . . . . . . . . . . . . . . 75

5.3 The precession angle $\Phi$ vs. the precession field ( $\left.\int \mathrm{Bdl}\right)$ for the three polarized data samples from this measurement. The best fit line (constrained through 0$)$, which corresponds to the first order $(n=0)$ solution. . . . . 77

5.4 The precession angle $\Phi$ vs. the precession field $\left(\int \mathrm{Bd}\right)$ for the three polarized data samples from this measurement and the two points from the previous measurement (E756). The best fit line, which corresponds

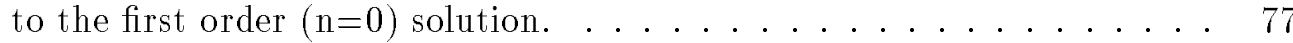

5.5 The magnetic moment results vs. momentum for the neutral production sample with $\int \mathrm{Bdl}=-24.36 \mathrm{~T} \cdot \mathrm{m}$ and the momentum averaged results for the four data samples. . . . . . . . . . . . . . 79

5.6 The $\mathrm{x}, \mathrm{y}$, and $\mathrm{z}$ asymmetry histograms for the neutral production $\Omega^{-}$ sample at $\int \mathrm{Bdl}=-24.36 \mathrm{~T} \cdot \mathrm{m}$. The lines show the asymmetry result from the hybrid monte carlo analysis. . . . . . . . . . . . 88

6.1 Comparison of experimental measurements of baryon magnetic moments with the theory which provides the best $\mu_{\Omega^{-}}$value. The $\mathrm{y}$-axis is the difference between theory and experiment in units of nuclear magnetons. The two cases include two conditions on the parameters used in this model. 93 
Table of Contents 


\section{List of Figures}




\section{List of Tables}




\section{Chapter 1}

\section{Introduction}

\subsection{Magnetic Moments and Baryon Structure}

The magnetic moment of a particle is an intrinsic property, which is determined by the particle's structure. For a pointlike particle, with spin $\vec{S}$ the magnetic moment, $\vec{\mu}$, is particularly simple,

$$
\vec{\mu}=\frac{e}{m c} \vec{S}
$$

where $\mathrm{e}$ is the particles' charge, and $\mathrm{m}$ its mass. If the particle has some internal structure, the magnetic moment is more complicated.

$$
\overrightarrow{\mu_{b}}=\sum_{i}\left\langle\Phi_{b}\left|\frac{e_{i}}{2 m_{i} \hbar c\left(J_{i} / \hbar+1\right)}\left(g_{s} \vec{S} \cdot \vec{J}+g_{l} \vec{L} \cdot \vec{J}\right)_{i} \frac{\vec{S}_{i}}{\left|\overrightarrow{S_{i}}\right|}\right| \Phi_{b}\right\rangle,
$$

where $\mathrm{e}_{i}, \mathrm{~m}_{i}, \vec{L}_{i}, \vec{S}_{i}$, and $\vec{J}_{i}$ are the ith constituent particle's charge, mass, orbital angular momentum, intrinsic spin angular momentum, and total angular momentum. The parameters $g_{l}$ and $g_{s}$ depend on the interactions of the constituent particles and are known as the gyromagnetic ratio and spin-orbit coupling. The $\left|\Phi_{b}\right\rangle$ are the spin-flavor wave functions of the constituent particles. 
Measuring the magnetic moments provides a "window" into the structure of composite particles. Historically measuring the neutron and proton magnetic moments gave early evidence supporting the quark model of hadrons. The non-zero magnetic moment of the neutron indicated that it was not a pointlike particle. The measurement of the ratio of the proton and neutron magnetic moments gave the ratio of the charges of the up and down quarks.

In the quark model, baryons are made up of three valence quarks with charge $-1 / 3 \mathrm{e}$ or $+2 / 3 \mathrm{e}$, where $\mathrm{e}$ is the magnitude of the electron charge. The quarks are bound by the exchange of gauge bosons called gluons. The formalism used to describe the gluon-quark interaction is called quantum-chromodynamics (QCD).

Present models of baryon structure used to predict magnetic moments can be categorized as either depending on the fundamental symmetry of the quark wavefunctions within baryons, symmetry based models, or models based on the interaction of the quarks within the baryons, QCD based models.

\subsubsection{Symmetry Based Models}

In the static quark model (SQM) the quarks in a baryon are considered to be in a $\mathrm{L}=0$ state with negligible electromagnetic interaction and no relativistic corrections. Because quarks are fermions they must be antisymmetric under color exchange. Therefore the spin-flavor wavefunctions must be symmetric. These wavefunctions are given by the $\mathrm{SU}(6)$ symmetry group and are shown in Table 1.1

If the magnetic moment of a baryon is given by sum of the $\vec{\mu}_{i}$, the individual valence quark moments, the magnetic moments can be calculated using the operator

$$
\vec{\mu}_{b}=\left\langle\Phi_{b}\left|\sum_{i} \vec{\mu}_{i}\right| \Phi_{b}\right\rangle
$$

on the wavefunctions in Table 1.1. Table 1.2 gives the expressions for the baryon magnetic moments in terms of the quark moments. Given the up, down, and strange 


\begin{tabular}{c|c} 
Baryon & Quark wavefunctions \\
\hline$p \uparrow$ & $\sqrt{2 / 3} u \uparrow u \uparrow d \downarrow-\sqrt{1 / 6}(u \uparrow u \downarrow+u \downarrow u \uparrow) d \uparrow$ \\
$n \uparrow$ & $\sqrt{2 / 3} d \uparrow d \uparrow u \downarrow-\sqrt{1 / 6}(d \uparrow d \downarrow+d \downarrow d \uparrow) u \uparrow$ \\
$\Lambda \uparrow$ & $\sqrt{1 / 2}(u \uparrow d \downarrow-u \downarrow d \uparrow) s \uparrow$ \\
$\Sigma^{+} \uparrow$ & $\sqrt{2 / 3} u \uparrow u \uparrow s \downarrow-\sqrt{1 / 6}(u \uparrow u \downarrow+u \downarrow u \uparrow) s \uparrow$ \\
$\Sigma^{0} \uparrow$ & $\sqrt{2 / 3} u \uparrow d \uparrow s \downarrow-\sqrt{1 / 6}(u \uparrow d \downarrow+u \downarrow d \uparrow) s \uparrow$ \\
$\Sigma^{-} \uparrow$ & $\sqrt{2 / 3} d \uparrow d \uparrow s \downarrow-\sqrt{1 / 6}(d \uparrow d \downarrow+d \downarrow d \uparrow) s \uparrow$ \\
$\Xi^{0} \uparrow$ & $\sqrt{2 / 3} s \uparrow s \uparrow u \downarrow-\sqrt{1 / 6}(s \uparrow s \downarrow+s \downarrow s \uparrow) u \uparrow$ \\
$\Xi^{-} \uparrow$ & $\sqrt{2 / 3} s \uparrow s \uparrow d \downarrow-\sqrt{1 / 6}(s \uparrow s \downarrow+s \downarrow s \uparrow) d \uparrow$ \\
$\Omega^{-} \uparrow$ & $s \uparrow s \uparrow s \uparrow$
\end{tabular}

Table 1.1: The baryon spin-flavor SU(6) wavefunctions.

quark moments, obtained by measuring the magnetic moments of any three baryons such as the proton, neutron, and $\Lambda$ magnetic moments, it is possible to find numerical values for the baryon magnetic moments under SQM. Table 1.2 gives these predictions and previous experimental values of $\mu_{\text {hyperon }}$. The $\Sigma^{0}-\Lambda$ is a transition moment which can be predicted by the overlap of the $\Sigma^{0}$ and $\Lambda$ wavefunctions from

$$
\mu_{\Sigma-\Lambda}=\left\langle\Phi_{\Sigma}\left|\sum_{i} \vec{\mu}_{i}\right| \Phi_{\Lambda}\right\rangle
$$

\begin{tabular}{c|l|c|c} 
Hyperon & $\mu_{\text {hyperon }}$ in Terms of $\mu_{\text {quark }}$ & $\mathrm{SU}(6)$ predictions & Experimental Result \\
\hline proton & $\mu_{\text {proton }}=4 / 3 \mu_{u}-1 / 3 \mu_{d}$ & Input & $2.793[1]$ \\
neutron & $\mu_{\text {neutron }}=4 / 3 \mu_{d}-1 / 3 \mu_{u}$ & Input & $-1.913[1]$ \\
$\Lambda$ & $\mu_{\Lambda}=\mu_{s}$ & Input & $-0.613 \pm 0.004[2]$ \\
$\Sigma^{+}$ & $\mu_{\Sigma^{+}}=4 / 3 \mu_{u}-1 / 3 \mu_{s}$ & 2.74 & $2.4613 \pm 0.0034 \pm 0.0040[3]$ \\
$\Sigma^{0}-\Lambda$ & $\left(\mu_{d}-\mu_{u}\right) / \sqrt{3}$ & -1.63 & $-1.61 \pm 0.08[4]$ \\
$\Sigma^{-}$ & $\mu_{\Sigma^{-}}=4 / 3 \mu_{d}-1 / 3 \mu_{s}$ & -1.21 & $-1.156 \pm 0.014[5]$ \\
$\Xi^{0}$ & $\mu_{\Xi}=4 / 3 \mu_{s}-1 / 3 \mu_{u}$ & -1.46 & $-1.23 \pm 0.014[6]$ \\
$\Xi^{-}$ & $\mu_{\Xi-}=4 / 3 \mu_{s}-1 / 3 \mu_{d}$ & -0.52 & $-0.6505 \pm 0.0025[7]$ \\
$\Omega$ & $\mu_{\Omega^{-}}=3 \mu_{s}$ & -1.83 & $-1.94 \pm 0.16 \pm 0.14[8]$
\end{tabular}

Table 1.2: Static quark model predictions. The experimental results are also listed for comparison.

The agreement in Table 1.2 is quite good considering the simplicity of the assumptions used in formulating SQM. However it is clear that further corrections to SQM are 
necessary to make predictions to the accuracy of current measurements. Various authors have tried to improve this agreement by augmenting SQM. Some of the corrections to SQM are:

- orbital angular momentum in the quark wavefunctions $[9,10]$

- quark mass effects $[11,12,13,14]$

- charge and charge shielding effects [15]

- relativistic effects $[16,17]$

- configuration mixing and symmetry breaking $[18,19,20]$

- pion interactions [21]

Another method of calculating magnetic moments, known as sum rules, is an attempt to cancel the effects listed above. The goal is to find "rules" relating the baryon moments to the moments by considering the similarities in quark content and the symmetry of the wavefunctions of various baryons. Originally these rules were obtained by comparing the masses and quark contents of various baryons under SU(3) [22, 23, 24]. However, they have since been extended to other symmetry groups specifically the SU(6) symmetry group $[25,26,27]$.

A typical sum rule prediction is calculated using the isospin symmetry group and applying the electromagnetic Lagrangian to the states in this symmetry group. By calculating the electromagnetic interaction in the limit of zero momentum transfer, $\mathrm{q}^{2} \rightarrow 0$, the magnetic moment terms for each baryon can be obtained and compared. In the case of [22] this yields the following relations:

$$
\begin{gathered}
\mu_{\Sigma^{+}}=\mu_{\text {proton }} \\
\mu_{\Lambda}=\frac{1}{2} \mu_{\text {neutron }}
\end{gathered}
$$




$$
\begin{gathered}
\mu_{\Xi^{0}}=\mu_{\text {neutron }} \\
\mu_{\Xi^{-}}=\mu_{\Sigma^{-}}=-\left(\mu_{\text {proton }}+\mu_{\text {neutron }}\right) \\
\mu_{\Sigma^{0}}=-\frac{1}{2} \mu_{\text {neutron }} \\
\mu_{\Sigma^{0}-\Lambda}=\frac{\sqrt{3}}{2} \mu_{\text {neutron }}
\end{gathered}
$$

The numerical predictions for these equations and the other sum rule predictions for the baryon magnetic moments are shown in Table 1.3

\begin{tabular}{c|c|c|c|c|c} 
& \multicolumn{5}{|c}{ Sum Rule Reference } \\
Hyperon & {$[22]$} & {$[23]$} & {$[24]$} & {$[25]$} & {$[26]$} \\
\hline proton & input & input & input & 3.04 & 3.0 \\
neutron & input & -1.85 & - & & -1.79 \\
-2.0 & & & & & \\
$\Lambda$ & -0.96 & -0.93 & -0.61 & -0.50 & -0.7 \\
$\Sigma^{+}$ & 2.79 & 2.79 & - & 2.73 & 2.4 \\
$\Sigma^{0} \Lambda$ & -1.65 & - & - & - & -1.55 \\
$\Sigma^{-}$ & -0.88 & -0.93 & - & -1.26 & -1.0 \\
$\Xi^{0}$ & -1.91 & -1.86 & - & -1.32 & -1.4 \\
$\Xi^{-}$ & -0.88 & -0.93 & - & -0.93 & -0.9 \\
$\Omega^{-}$ & - & -2.79 & - & - & -
\end{tabular}

Table 1.3: Magnetic moment predictions for various sum rule calculations.

The sum rule predictions agree with experimentally determined values of the moments from Table 1.2 in only a few cases. For the most part the predictions are not an improvement over the static quark model predictions.

\subsubsection{QCD based models}

QCD is currently the best theory governing the interactions between quarks. By considering these interactions and the interactions of the individual quarks with a weak external magnetic field one can calculate the behavior of the system of quarks in the magnetic field and thus calculate the magnetic moments. QCD calculations are made difficult by the divergent nature of the color force. At small distances, when quarks 
are deep in the QCD potential well, they behave as free particles. However, at large distances the potential due to the color interaction increases with distance. The large potential at large distances makes creation of quark anti-quark pairs energetically favorable leading to confinement.

Another way to understand the confinement of quarks is to consider the coupling of the strong force, $\alpha_{s}$, which is a function of the momentum transfer, $\mathrm{q}^{2}$. At large $\mathrm{q}^{2}$, a situation which corresponds to small distances, $\alpha_{s}$ is small $\left(\alpha_{s}<1\right)$. However, for small values of $\mathrm{q}^{2}$, i.e. large distances, $\alpha_{s}$ is of order one. Thus a full treatment of the QCD Lagrangian at small momentum transfers, such as those involved in measuring a magnetic moment, involves calculating an infinite number of diagrams, which makes a full treatment impossible. The two QCD based models discussed here differ in the treatment of quark confinement in the baryon and in how they avoid the non-perturbative nature of QCD.

Bag models treat composite particles as bubbles of quark gas in vacuum. The first bag models were calculated under the assumption of massless, non-interacting, free quarks within a spherical boundary $[28,29]$. The magnetic moment is calculated via the energy shift from interaction with a weak, constant magnetic field. This model has also been expanded to include relativistic effects [30], pion and gluon interactions [31, 32], and non-spherical bag potentials [33]. The magnetic moment predictions for the bag model and the various corrections are listed in Table 1.4.

Even though the bag model uses more sophisticated assumptions the predictions are not particularly impressive. Compared to the static quark model predictions and the measured values in Table 1.2 the results of the bag model are not much better than those of SQM.

Recently lattice gauge theory (LGT) has been used to calculate the magnetic moments and other intrinsic properties of the octet and decuplet baryons [34, 35]. LGT is a treatment using the QCD Lagrangian under the "quenched QCD" approximation, 


\begin{tabular}{c|c|c|c|c|c|c} 
& \multicolumn{6}{|c}{ Bag Model Reference } \\
Hyperon & \multicolumn{2}{|c|}{ simple bag model } & rel. bag & \multicolumn{2}{|c}{ pion, gluon int. } & non-spherical bag \\
& {$[28]$} & {$[29]$} & {$[30]$} & {$[31]$} & {$[32]$} & {$[33]$} \\
\hline proton & 2.60 & input & 2.60 & 2.65 & input & 2.618 \\
neutron & -1.73 & -1.85 & -1.55 & -1.91 & input & -1.913 \\
$\Lambda$ & - & -0.71 & -0.61 & -0.61 & input & -0.614 \\
$\Sigma^{+}$ & - & 2.70 & 2.42 & 2.34 & 2.46 & 2.475 \\
$\Sigma^{0}-\Lambda$ & - & 0.86 & - & - & 0.65 & \\
$\Sigma^{-}$ & - & -1.00 & -0.99 & -1.05 & -1.16 & -1.088 \\
$\Xi^{0}$ & - & -1.56 & -1.25 & -1.36 & -1.31 & -1.365 \\
$\Xi^{-}$ & - & -0.64 & -0.60 & -0.61 & -0.71 & -0.552 \\
$\Omega^{-}$ & - & - & - & - & -2.52 & -
\end{tabular}

Table 1.4: Magnetic moment predictions for various bag model calculations.

neglecting quark anti-quark $(q \bar{q})$ pairs, to model the baryons. The actual technique is to simulate the quark interactions numerically on a space-time lattice.

Calculations of magnetic moments under LGT depend on observing the effect of a constant weak magnetic field on the baryon propagators. Including a magnetic field B, changes the propagator $\mathrm{G}(\mathrm{t})$ to $[34]$

$$
G(t)=e^{-(m \pm \mu B) t}
$$

with $\mathrm{eBt} / \mathrm{m} \ll 1$, or

$$
G(t)=e^{-(m \pm \mu B+e B / 2 m) t}
$$

with $\mathrm{eBt} / \mathrm{m} \gg 1$, where $\mathrm{e}, \mathrm{m}, \mu$ are the charge, mass, and magnetic moment of the quark, and $t$ is the time. The \pm depends on whether the quark is spin antialigned or spin aligned with the magnetic field.

The energy difference between spin-aligned and spin-antialigned states is calculated after the baryon has come to equilibrium. There is a zero-field correction, $\mathrm{m}_{ \pm}$, even if $\mathrm{B}=0$, since for a finite number of configurations there is a splitting between the two states. The magnetic moment is given by 


$$
\mu=\frac{e g s}{2 m \hbar}
$$

with

$$
g=\left[m_{+}\left(E_{+}-m_{+}\right)-m_{-}\left(E_{-}-m_{-}\right)\right] / e s B .
$$

where e, s, and $\mathrm{m}$ are the baryon's charge, spin, mass and $\mathrm{B}$ is the magnetic field magnitude. $E_{ \pm}$is the energy obtained from evaluating the Lagrangian for the spinaligned and spin-antialigned states. Table 1.5 shows magnetic moment values from two LGT calculations.

\begin{tabular}{c|c|c} 
& \multicolumn{2}{|c}{ LGT Reference } \\
Hyperon & {$[34]$} & {$[35]$} \\
\hline proton & $2.70 \pm 1.0$ & input \\
neutron & $-1.6 \pm 0.5$ & - \\
$\Sigma^{+}$ & - & $3.16 \pm 0.40$ \\
$\Sigma^{-}$ & - & $-2.50 \pm 0.29$ \\
$\Xi^{0}$ & - & $0.58 \pm 0.10$ \\
$\Xi^{-}$ & - & $-2.08 \pm 0.24$ \\
$\Omega^{-}$ & $-1.7 \pm 0.5$ & $-1.73 \pm 0.22$
\end{tabular}

Table 1.5: Magnetic moment predictions for lattice gauge calculations.

The use of faster computers allows the use of finer lattice spacings. This will hopefully make for more precise LGT calculations in the future.

Even with the more sophisticated models and corrections to SQM, a single model which can successfully predict the magnetic moments of the baryons to the level of experimental measurements does not exist. Such a model would provide evidence that the interaction between quarks and gluons confined within particles is well understood.

\subsection{The Omega Minus Magnetic Moment}

The $\Omega^{-}$is a particularly simple system, consisting of three spin aligned strange quarks. It has an intrinsic spin $(3 / 2) \hbar$, and a mass, $\mathrm{m}_{\Omega^{-}}$, of $1.672 \mathrm{GeV} / \mathrm{c}^{2}[36]$. 
To preserve the symmetry of the wavefunction shown in Table 1.1, these quarks must be in an even integer orbital-angular momentum state. The simplest, lowest mass state allowed in this configuration is the orbital angular momentum ground state $(\mathrm{L}=0)$. This configuration should make the $\Omega^{-}$a simple magnetic moment to evaluate under most models.

It should also be noted that the $\Omega^{-}$is made up of relatively massive strange quarks, making relativistic and mass corrections smaller compared to other ground state baryons. The state of three identical quarks lessens the possibility of configuration mixing in this system [37]. The $\Omega^{-}$system is relatively impervious to the complications of other baryons, making a precision measurement of the magnetic moment an interesting test of theory.

A list of predictions for the $\Omega^{-}$magnetic moment and the previously measured experimental value is in Table 1.6.

\begin{tabular}{l|c|c} 
Model & $\mu_{\Omega^{-}}$ & Reference \\
\hline SQM & -1.83 & Table 1.2 \\
SQM mass correction & -1.3 & {$[12]$} \\
SQM mass correction & $-1.52-1.48$ & {$[14]$} \\
SQM charge correction & -2.33 & {$[15]$} \\
Relativistic Bag Model & $-1.95-2.52$ & {$[30]$} \\
LGT & $-1.7 \pm .6$ & {$[34]$} \\
LGT & $-1.73 \pm .22$ & {$[35]$} \\
Measured value Fermilab E756 & $-1.94 \pm 0.17 \pm 0.14$ & {$[7]$}
\end{tabular}

Table 1.6: Theoretical predictions for the $\Omega^{-}$magnetic moment. The measured value is included for comparison

All the values for the various models are within $\pm 3 \sigma$ of the experimental measurement. A more precise measurement of the $\Omega^{-}$magnetic moment would allow a further test for these models in this simple system. It is clear from Table 1.2 that under SQM $\mu_{\Omega^{-}}=3 \mu_{\text {strange }}$, and therefore a measurement of sufficient accuracy could be used to evaluate the strange quark magnetic moment as a parameter for models and comparison to the value obtained from measurements of the other hyperons. 


\subsection{Magnetic Moments by the Spin Precession Tech- nique}

The magnetic moment of an object, $\vec{\mu}$, measures the strength of the object's interaction with an external magnetic field. Classically, an object in a magnetic field $\vec{B}$ undergoes an energy shift, $\Delta \mathrm{E}$

$$
\Delta E=-\vec{\mu} \cdot \vec{B},
$$

and experience a torque, $\vec{\tau}$,

$$
\vec{\tau}=\vec{\mu} \times \vec{B}
$$

where $\vec{\mu}$ is the particles' magnetic moment. In a constant magnetic field this torque gives rise to a precession of the angular momentum of the particle. In the case of a particle of spin $\vec{s}$ the rate of change of the direction of the spin vector is given by

$$
\frac{d \vec{s}}{d t}=\mu \vec{s} \times \vec{B}
$$

The above equation holds true in the rest frame of the particle that precesses. However a charged particle follows a curved path in a magnetic field. In this case the rest frame is also rotates with the particle. The time development of the spin vector $\vec{s}$ in a non rotating frame is given by

$$
\left(\frac{d \vec{s}}{d t}\right)_{\text {nonrotating }}=\left(\frac{d \vec{s}}{d t}\right)_{\text {rotating }}+\omega \times \vec{s}
$$

For a Galilean transformation $\omega$ is just the rotation frequency, but for a Lorentz transformation, $\omega$ is [38]

$$
\omega=\frac{\gamma^{2}}{\gamma+1} \frac{\vec{a} \times \vec{v}}{c^{2}}
$$


where $\mathrm{c}$ is the speed of light, $\vec{v}$ is the velocity of the particle, and $\vec{a}$ is the acceleration given by the Lorentz equation

$$
\vec{a}=\frac{q}{\gamma m c} \vec{v} \times \vec{B}
$$

where $\mathrm{m}$ is the particle's mass and

$$
\gamma=\frac{1}{\sqrt{1-\left(\frac{v}{c}\right)^{2}}}
$$

Substituting Equations 1.10, 1.12, and 1.13 into Equation 1.11 and assuming $\vec{v}-\vec{B}$ and $\mathrm{v} \cong \mathrm{c}$ gives:

$$
\frac{d \vec{s}}{d t}=\left[\mu+\frac{q}{m c}\left(-1+\frac{1}{\gamma}\right)\right] \vec{s} \times \vec{B}
$$

If the particles are produced so that the direction of $\vec{s}$ is in a plane perpendicular to $\vec{B}$, Equation 1.15 can be simplified to

$$
\frac{d \phi}{d t}=\left[\mu+\frac{q}{m c}\left(-1+\frac{1}{\gamma}\right)\right] B
$$

Since $\mathrm{v} \cong \mathrm{c}$, and therefore $\mathrm{dt}=\frac{d l}{c}$ we can find an expression for the precession angle $\phi$ in terms of the field integral,

$$
\phi=\left[\frac{\mu}{c}+\frac{q}{m c^{2}}\left(-1+\frac{1}{\gamma}\right)\right] \int B d l .
$$

This expression is for the total precession angle $\phi$. However, if we measure the spin direction with respect to the momentum direction of the particle, we must also account for the fact that the momentum vector of the particle has precessed through an angle $\Theta$ given by

$$
\Theta=\frac{q}{\gamma m c^{2}} \int B d l
$$


The angle through which the spin precesses relative to the particle's momentum direction, $\Phi$, is the difference between the total precession angle and the angle through which the momentum precesses which eliminates the dependence on $\gamma$.

$$
\Phi=\phi-\Theta=\left[\frac{\mu}{c}-\frac{q}{m c^{2}}\right] \int B d l
$$

With the discovery of polarization in $\Lambda$ hyperons produced at high energies [41] it became possible to make precise measurements of hyperon magnetic moments by the spin precession method. The precession angle can be measured by determining the initial and final polarization directions. The initial direction of the polarization was found to be be perpendicular to the plane formed by the incoming beam and the outgoing beam (the production plane) as required to conserve parity in the strong interaction.

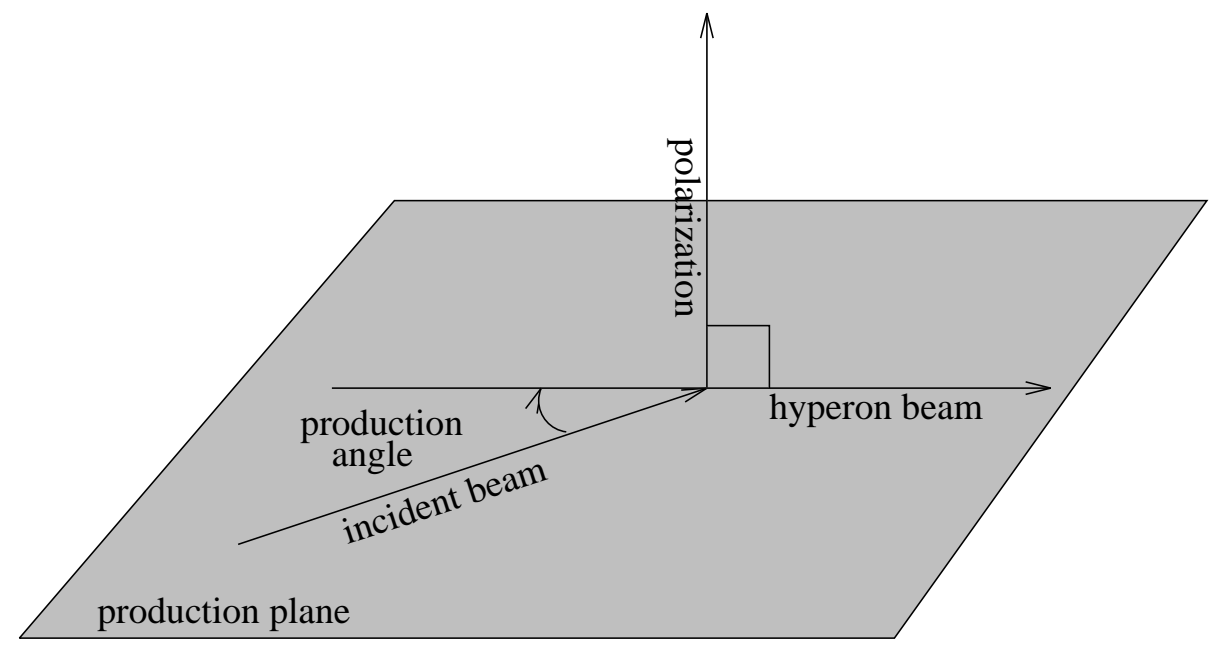

Figure 1.1: Producing polarized hyperons at a production angle.

This situation is illustrated in Figure 1.1. The final state polarization direction is determined by the decay distribution of the final decay products. If the initial polarization is along the $\mathrm{x}$-axis, and the magnetic field is along the $\mathrm{y}$-axis, then the precession angle, $\Phi$, is given by 


$$
\Phi=\tan ^{-1}\left(\frac{P_{z}}{P_{x}}\right)+n \pi
$$

where $P_{z}$ is the final z-component of the polarization, $P_{x}$ is the final state $\mathrm{x}$ component of the polarization, and $n$ is an integer taking into account the possibility of precession angles greater than $180^{\circ}$. This method has been used for most of the previous measurements of hyperon magnetic moments. [2, 3, 5, 6, 7, 8] 


\section{Chapter 2}

\section{The Apparatus}

There are two ways to produce polarized $\Omega^{-} \mathrm{s}-$ through spin transfer from a beam of polarized hyperons, or through some innate polarization from the production process by using unpolarized particles. We constructed a beamline which could run in either mode. For both of these modes a secondary neutral beam was targeted to produce a tertiary negative hyperon beam. This tertiary beam passed through a curved collimator embedded in a magnet $7.31 \mathrm{~m}$ in length. When run at maximum current it produced a field of 3.33 Tesla and a field integral of $24.36 \mathrm{~T} \cdot \mathrm{m}$. This magnet served three functions: it provided shielding for the experimental enclosure, selected the charge and momentum of the beam entering the spectrometer, and precessed the hyperon magnetic moment. The hyperons in the beam which survived through the collimator decayed in a spectrometer which tracked the decay products through a magnetic field to measure their momenta. These momenta gave the final polarization direction of the parent hyperon.

\subsection{The Primary Beam}

The data for this dissertation were taken by experiment E800 at Fermi National Accelerator Laboratory in Batavia, Illinois during the 1991-1992 fixed target run. The 
accelerator delivered $800 \mathrm{GeV} / \mathrm{c}$ protons at an intensity of $10^{13}$ protons per 20 second spill with a duty cycle of one minute. After leaving the accelerator the protons were routed into several beams and delivered to fixed target experiments.

E800 used the proton center beamline which transported the primary $800 \mathrm{GeV} / \mathrm{c}$ proton beam at intensities between $3.0 \times 10^{10}$ to $2.5 \times 10^{12}$ protons/spill. The beam was transported through enclosures PC1, PC2, which contained vertical and horizontal dipoles and vertical and horizontal focusing quadrople magnets. Finally, the beam entered the PC3 enclosure where it struck a target.

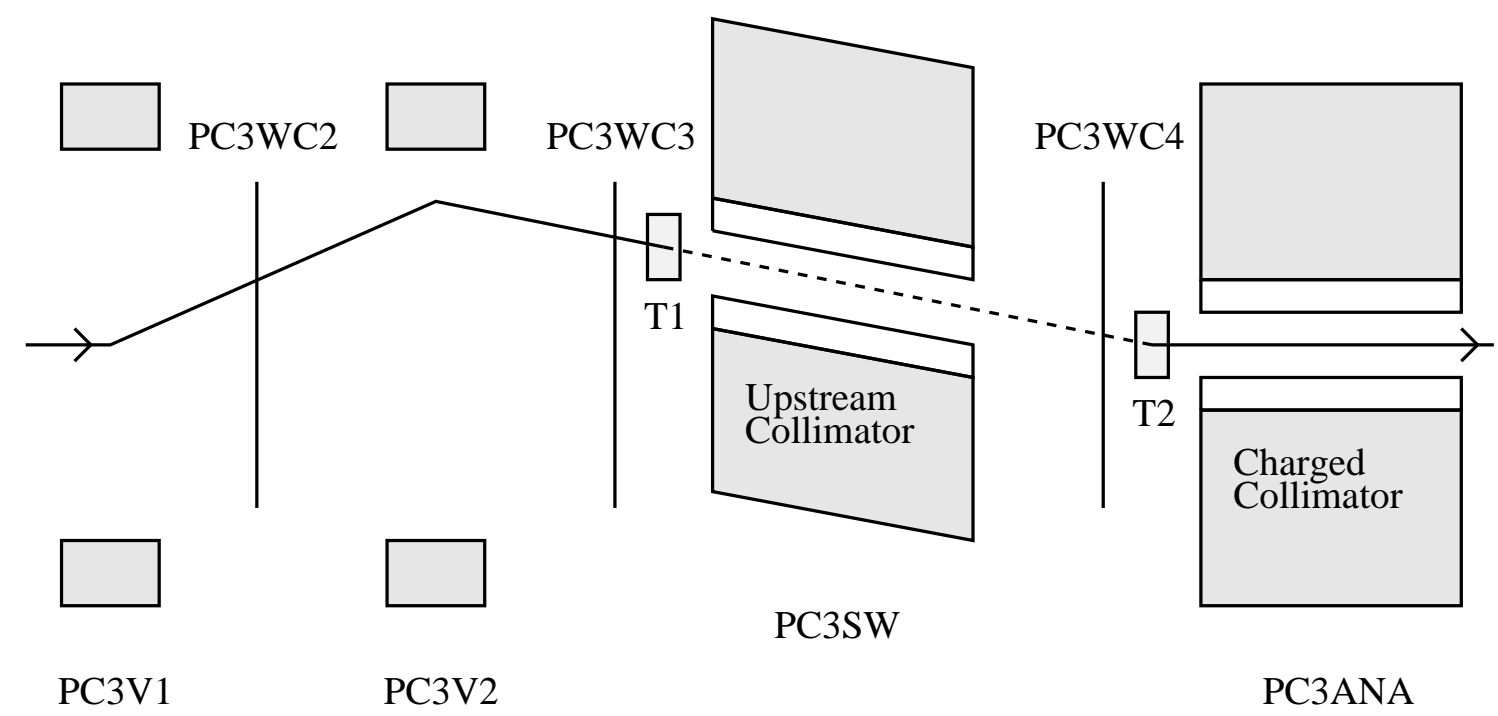

Figure 2.1: Schematic of the PC3 enclosure (elevation view) including magnets, targets (T1 and T2) and beam SWIC's (PC3WC2, PC3WC3, and PC3WC4

\subsection{Secondary and Tertiary Beams}

To produce a negative hyperon beam of maximum polarization and maximum intensity, the beamline allowed many targeting configurations. Electronically controlled hydraulic jacks, target movers and magnet controls allowed targeting angles to be changed from 
the experiment control room.

The beamline was designed to bring a focused low angular divergence proton beam (less than $2 \mathrm{~mm}$ in diameter) to the upstream target and to transport a neutral secondary beam to the downstream target. The low divergence primary beam gave a low divergence secondary beam. This configuration was designed to maximize the transmission of the tertiary negative hyperon beam through the charged collimator.

A schematic of the PC3 enclosure is shown in Figure 2.1. PC3 housed the dipoles PC3V1, PC3V2 and PC3H, the targets and both collimator channels, the neutral collimator embedded in PC3SW and the charged collimator contained in the precession magnet PC3ANA. The magnets were controlled via the Fermilab Epicure beamline control software and online CAMAC. The position of the beam was determined using several segmented wire chambers (SWICs) with either $1 \mathrm{~mm}$ or $2 \mathrm{~mm}$ pitch. The SWICs produced a histogram of particle positions on displays in the control room.

\subsubsection{Targeting Dipoles}

A string of three dipoles, PC3V1 was used to lower or raise the beam. In order to strike the targets at various production angles the beam was brought back on either the upstream or downstream target by another set of vertical dipoles PC3V2.

\subsubsection{The Targets}

E800 used two Beryllium targets. Beryllium as a target material gives fewer secondary nuclear interactions within the targets than denser targets and is still dense enough to provide adequate production of hyperons. Beryllium targets give a higher secondary energy spectrum with a slightly higher polarization signal, since the polarization signal is not diluted by secondary interactions within the target. The minimal secondary interactions also yields higher energy hyperon beams which maximizes the transmission of the tertiary hyperon beam to the experimental hall. 
The upstream target was mounted with its center $12.1 \mathrm{~cm}$ in front of the neutral collimator entrance and centered on the collimator axis. This target was a $6.5 \mathrm{~mm}$ diameter rod $15.72 \mathrm{~cm}$ long. The downstream target was mounted with its center $62.2 \mathrm{~cm}$ downstream of the neutral collimator and $13.2 \mathrm{~cm}$ in front of the charged collimator entrance and consisted of a $5.15 \times 5.28 \times 141 \mathrm{~mm}^{3}$ rod centered on the charged collimator axis. These targets were mounted in electronically controlled target carriers, which allowed target positioning to $0.1 \mathrm{~mm}$. The carriers also allowed the targets to be removed from the beam.

\subsubsection{The Neutral Collimator}

The neutral collimator (Figure 2.2) formed a secondary neutral beam and served as a beam dump for the primary proton beam. It was made of brass and tungsten segments and was $640 \mathrm{~cm}$ long with a defining aperture $2.54 \times 2.54 \times 914.4 \mathrm{~mm}^{3}$. The defining aperture was $396.24 \mathrm{~cm}$ downstream from the upstream end of the magnet. This collimator was embedded in a $6.0 \mathrm{~m}$ long $\mathrm{B} 2$ main ring dipole with a horizontal field, which dumped the beam in either the upper or lower tungsten portion of the collimator and reduced background muons from entering the experimental hall. This magnet developed a field of 1.8 Tesla at a current of 5000 amps. The field was oriented perpendicular to the production plane at TGT1. Using this orientation the field cannot precess to neutral beam polarization since it is either parallel or anti parallel to the beam polarization. The front and rear of the magnet was mounted on adjustable, electronically controlled jacks to allow the secondary neutral beam to strike the downstream target at nonzero production angles. The jacks were located $55.1 \mathrm{~cm}$ from the upstream end of the magnet and $51.4 \mathrm{~cm}$ from the downstream end. The channel angle was thus controlled electronically by a CAMAC interface to the Fermilab Epicure system. The motor controls had 29,400 and 34,500 counts per inch respectively, which allowed precise and repeatable positioning of the magnet to better than $0.05 \mathrm{mrad}$. 


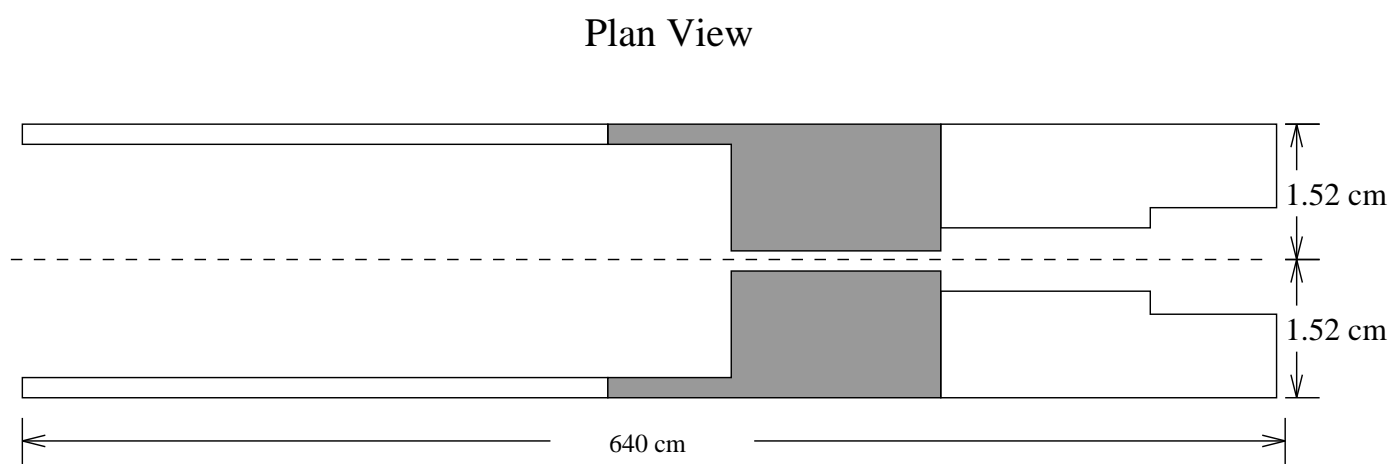

Elevation View

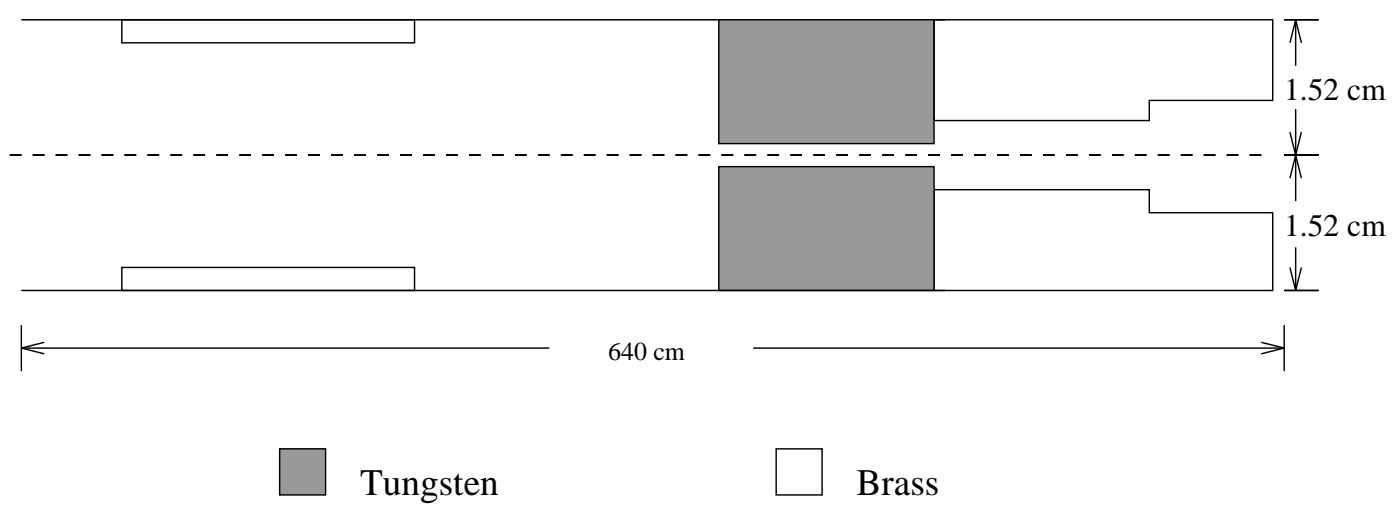

Figure 2.2: The E800 Neutral Collimator 


\subsubsection{The Precession Magnet and Charged Collimator}

The precession magnet was a 7.3 meter long dipole, PC3ANA, which had a magnetic field in the vertical direction. A collimator curved in the horizontal plane was embedded in this magnet to select a negatively charged beam.

Table 2.1 shows the field integral, $\int \mathrm{Bdl}$, of PC3ANA at two currents. Figure 2.3 shows the field integral with respect to the precession magnet current, the data for this figure was taken from the E756 field versus current measurements. Since the precession magnet had a sizeable fringe field it was necessary to measure the field along the entire path of the particle to calculate the field integral. This measurement was done by Fermilab E756 with a Hall probe at one or two inch intervals. The Hall probe was calibrated against a Nuclear Magnetic Resonance probe. Based on the calibration data the uncertainty of the field integral measurement was about 1\% [39]. As a check of the E756 measurements the field integral was calculated by comparing the cascade magnetic moment measured by E 800 to the previously measured value.

\begin{tabular}{|c|c|c|}
\hline Current $($ Amps $)$ & Field Integral $(\mathrm{T} \cdot \mathrm{m})$ & Central Orbit Momentum $(\mathrm{GeV} / \mathrm{c})$ \\
\hline 750 & $17.39 \pm 0.17$ & 285 \\
\hline 2900 & $24.36 \pm 0.24$ & 393 \\
\hline
\end{tabular}

Table 2.1: The $\int B d l$ and central orbit momentum for the 2 currents used in the sweeping magnet.

The charged collimator is shown in Figure 2.4. It was constructed from $2430.48 \mathrm{~cm}$

brass sections. The defining aperture was $5.08 \mathrm{~cm} \times 5.08 \mathrm{~mm}^{2}$, with bend angle of $18.37 \mathrm{mrad}$, and a central orbit radius of 38.83 meters. For most of the data taking period the magnet ran at a current of 2900 amperes with a central orbit momentum of $393 \mathrm{GeV} / \mathrm{c}$.

\subsection{Operating Modes}

E800 tried several methods to generate a polarized beam. The first and easiest of these was direct proton production as shown in figure 2.4a. This mode of production has 


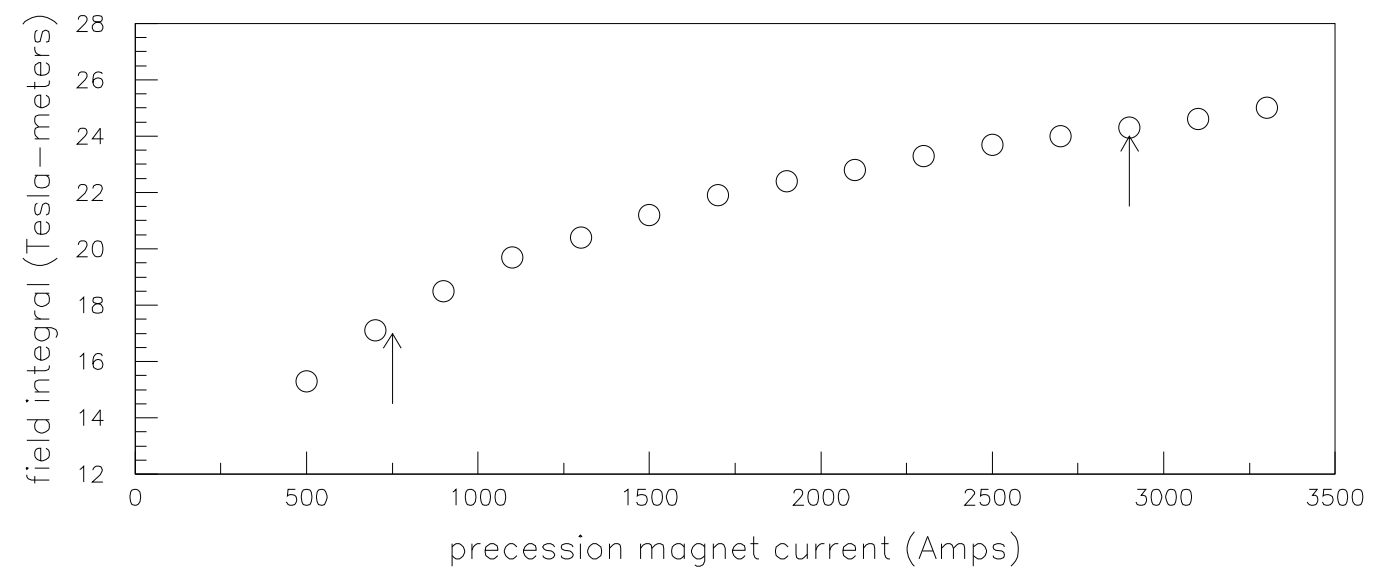

Figure 2.3: The field integral of the precession magnet versus the magnet current. These measurements were made by fermilab experiment E756.

been used in previous experiments [40] and produced only unpolarized $\Omega^{-}$hyperons. In this mode there was no neutral secondary beam, and the $800 \mathrm{Gev}$ proton beam struck TGT2 at a production angle and created a negatively charged secondary beam. The primary proton beam was bent in the vertical plane by the dipoles PC3V1 and PC3V2 to give a production angle. In order to allow transmission of the protons to TGT2 the upstream target was removed and the current in the sweeper dipole PC3SW was set to zero. It was also necessary to align PC3SW for maximum transmission of the beam to the second target, which we did with the remote controlled jacks at either end of the magnet. Data were taken at production angles of $\pm 1.8 \mathrm{mrad}$ and $0 \mathrm{mrad}$.

In the second mode, which we called spin transfer production, the proton beam struck TGT1 at a vertical production angle. The secondary neutral beam of neutrons, $\Lambda, \mathrm{K}^{0}$, $\gamma$, and $\Xi^{0}$ hyperons came though PC3SW at maximum current with the channel in the plane of the charged collimator. The neutral hyperons ( $\Lambda \mathrm{s}$, and $\left.\Sigma^{0} \mathrm{~s}\right)$ in the secondary beam were polarized. This polarization has been measured in previous experiments, 


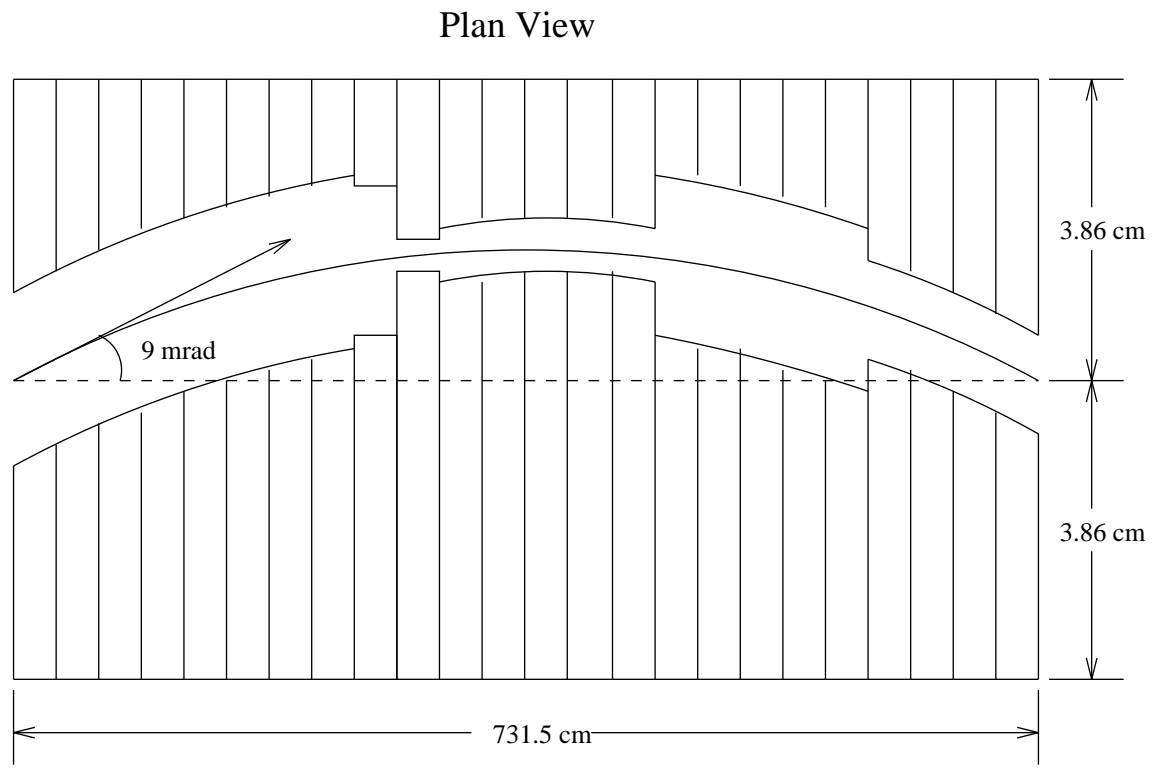

Elevation View

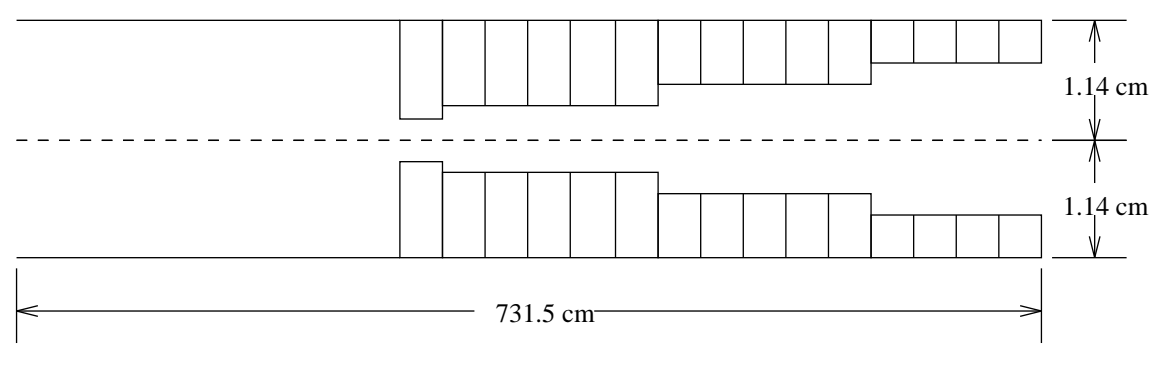

Brass

Figure 2.4: The E800 Charged Collimator 
and is perpendicular to the production plane. [41, 42, 43] It is not known if the neutrons are polarized. The horizontal field of PC3SW did not precess the horizontal polarization of the neutral hyperons since its field was parallel to the spin direction. The polarized neutral beam was targeted on TGT2 at angles of $\pm 1.8 \mathrm{mrad}$ and $0 \mathrm{mrad}$ to create a tertiary beam which passed through the charged collimator embedded in PC4ANA, giving a negatively charged beam. This mode is shown in Figure 2.4b.

The third mode, was to make an unpolarized neutral beam at TGT1, as diagrammed in Figure 2.4c. We knew this beam was unpolarized since it was produced at $0 \mathrm{mr}$. This beam passed though the neutral channel with PC3SW at maximum current and struck TGT2 at a production angle, similar to proton production but with a neutral secondary beam substituted for the proton beam. The tertiary beam then passed through the charged collimator which selected negatively charged particles. Most of the data for this measurement was taken in this mode at production angles of $\pm 1.8 \mathrm{mrad}$.

\subsection{The Charged Particle Spectrometer}

\subsubsection{General Design of the Spectrometer}

The charged particle spectrometer was designed to measure the position and momenta of the charged particles associated with both the decay sequences $\Omega^{-} \rightarrow \Lambda \mathrm{K}^{-}, \Lambda \rightarrow \mathrm{p} \pi^{-}$, and, $\Xi^{-} \rightarrow \Lambda \pi^{-}, \Lambda \rightarrow \mathrm{p} \pi^{-}$. The spectrometer coordinate system was right handed, with the $\mathrm{z}$-axis along the central beam axis. The $\mathrm{y}$-axis pointed up and the $\mathrm{x}$-axis was defined by the cross product of the $y$-axis into the z-axis. The origin of the coordinate system was at the center of the charged collimator exit. A schematic of the apparatus is shown in Figure 2.6.

This spectrometer consisted of 8 SSD planes (silicon strip detectors), 4 scintillation counters, 24 MWPC planes (multi-wire proportional chambers), and 2 momentum analysing magnets (see table 2.2 and figure 2.5 ). The analysis magnets were "BM109" 
a)

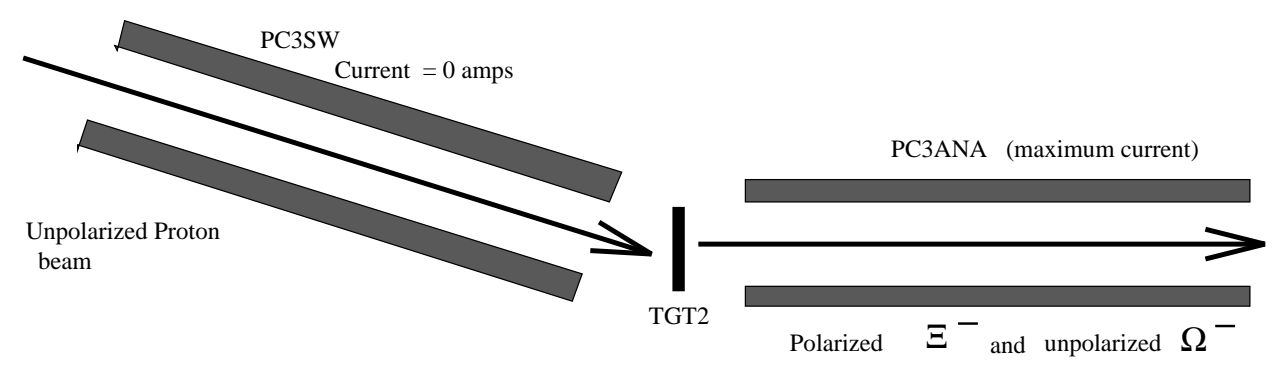

b)

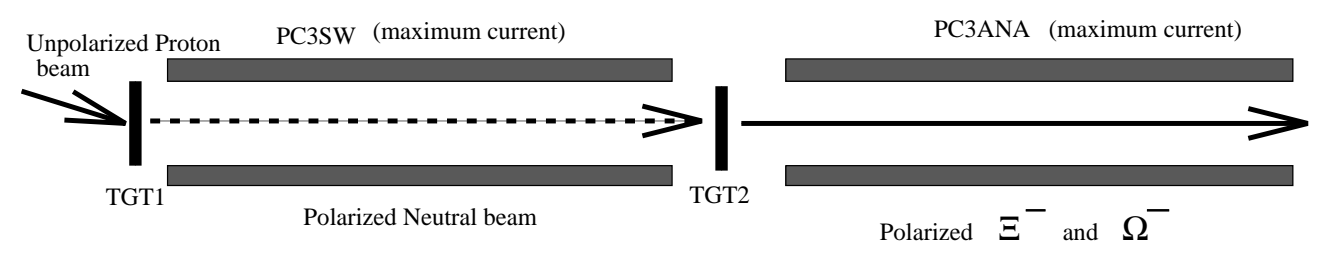

c)

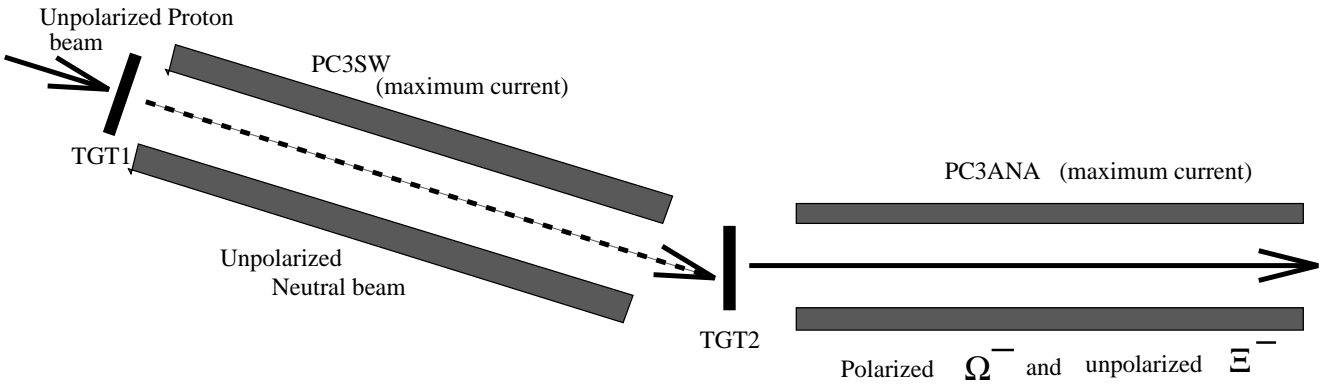

Figure 2.5: Operating Modes 
(bending magnets 109 inches long) dipole magnets, with the fields oriented vertically so charged particles were bent horizontally. In order to reduce the interactions of the particles, we put helium bags and tubes between the detectors in the apparatus. Us-

ing helium instead of air reduced the material in the spectrometer from $2.52 \times 10^{-1}$ to $9.55 \times 10^{-2}$ radiation lengths.

The high resolution detectors, the SSDs, determined the parent track. The $1 \mathrm{~mm}$ pitch MWPCs primarily tracked the $\mathrm{K}^{-}\left(\pi^{-}\right)$from the $\Omega^{-}\left(\Xi^{-}\right)$to determine the vertex location. In addition the $1 \mathrm{~mm}$ MWPCs helped resolve particle tracks upstream for three track events and improved the angular resolution of the decay product distributions. The $2 \mathrm{~mm}$ MWPCs were placed downstream to determine the decay product momenta.

\subsubsection{The Scintillation Counters}

E800 used four scintillation counters (S1, S2, V1, V2) for triggering on the presence of charged particles in the spectrometer. The scintillators $\mathrm{S} 1$, and $\mathrm{S} 2$ were $0.32 \mathrm{~cm}$ thick doped polystyrene. Pulses in these scintillators indicated a particle passing through the spectrometer. V1 and V2 were $0.32 \mathrm{~cm}$ thick plastic scintillators positioned outside of the beam aperture. A signal from V1 or V2 indicated a particle, or shower of particles outside of the nominal beam. The dimensions of these counters are listed in Table 2.2. The high voltage settings and efficiencies of these counters are listed in Table 2.3. V1 and V2 were built using two pieces of scintillator with a hole to allow the beam to pass through the center. This necessitated using two photomultiplier tubes and bases to give even coverage of both sides of the beam aperture. Each side was discriminated using NIM electronics. These signals were used in the trigger, and also latched and readout to the data aquisition. 


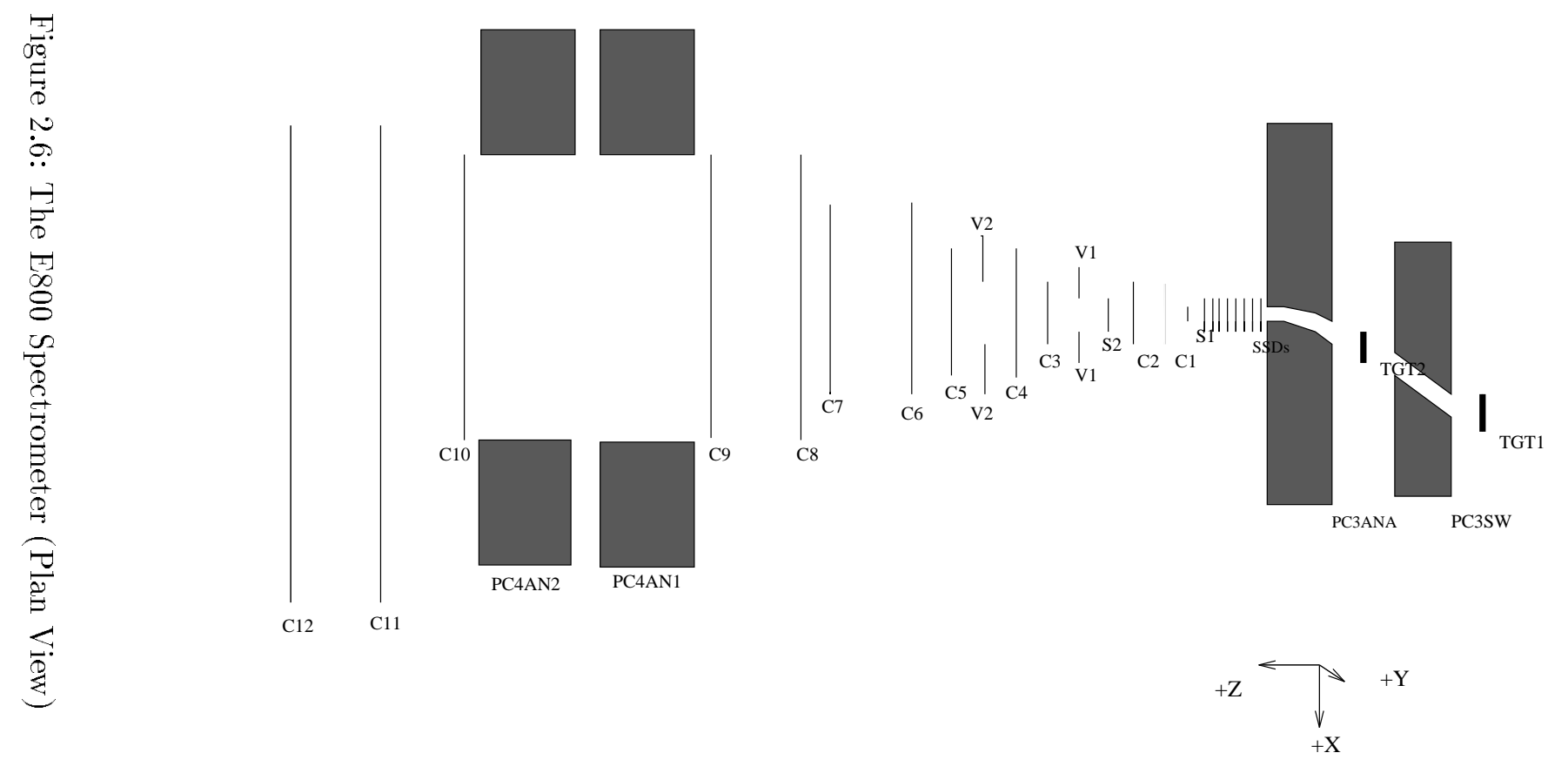




\begin{tabular}{|c|c|c|c|c|c|}
\hline Detector & $\begin{array}{l}\text { Z-Position } \\
(\mathrm{cm})\end{array}$ & $\begin{array}{c}\text { Dimensions } \\
\mathrm{x}(\mathrm{cm}) \times \mathrm{y}(\mathrm{cm})\end{array}$ & $\begin{array}{l}\text { Thickness } \\
\text { (cm) }\end{array}$ & $\begin{array}{l}\text { Pitch } \\
(\mathrm{mm})\end{array}$ & Device Type \\
\hline SSD1(x) & 74.43 & $2.8 \times 2.8$ & .0300 & .10 & SSD \\
\hline SSD2(y) & 79.22 & $2.8 \times 2.8$ & .0300 & .10 & SSD \\
\hline $\operatorname{SSD3}(\mathrm{x})$ & 100.97 . & $2.8 \times 2.8$ & .0300 & .10 & SSD \\
\hline SSD4(y) & 109.97 & $2.8 \times 2.8$ & .0300 & .10 & SSD \\
\hline SSD5(x) & 129.46 & $2.8 \times 2.8$ & .0300 & .10 & SSD \\
\hline SSD6(y) & 137.80 & $2.8 \times 2.8$ & .0300 & .10 & SSD \\
\hline SSD7(x) & 158.43 & $2.8 \times 2.8$ & .0300 & .10 & SSD \\
\hline SSD8(y) & 166.29 & $2.8 \times 2.8$ & .0300 & .10 & SSD \\
\hline S1 & 360.0 & $6.35 \times 3.81$ & .32 & $\mathrm{n} / \mathrm{a}$ & Scintillation \\
\hline $\mathrm{C} 1(\mathrm{x}, \mathrm{y})$ & 560.45 & $12.8 \times 12.8$ & $n / a$ & 1.0 & MWPC \\
\hline $\mathrm{C} 2(\mathrm{x}, \mathrm{y})$ & 775.41 & $12.8 \times 12.8$ & $n / a$ & 1.0 & MWPC \\
\hline V1 & 800.0 & $32.38 \times 8.89$ & .32 & $n / a$ & Scintillation \\
\hline V1(hole) & 800.0 & $11.43 \times 6.35$ & .32 & $\mathrm{n} / \mathrm{a}$ & $\mathrm{n} / \mathrm{a}$ \\
\hline $\mathrm{S} 2$ & 800.0 & $10.79 \times 6.35$ & .32 & $\mathrm{n} / \mathrm{a}$ & Scintillation \\
\hline $\mathrm{C} 3(\mathrm{x}, \mathrm{y})$ & 987.35 & $12.8 \times 12.8$ & $n / a$ & 1.0 & MWPC \\
\hline $\mathrm{V} 2$ & 1020.0 & $41.91 \times 11.43$ & .32 & $\mathrm{n} / \mathrm{a}$ & Scintillation \\
\hline V2(hole) & 1020.0 & $13.97 \times 8.25$ & .32 & $\mathrm{n} / \mathrm{a}$ & $\mathrm{n} / \mathrm{a}$ \\
\hline $\mathrm{C} 4(\mathrm{x}, \mathrm{y})$ & 1510.35 & $25.6 \times 25.6$ & $n / a$ & 1.0 & MWPC \\
\hline $\mathrm{C} 5(\mathrm{x}, \mathrm{y})$ & 2008.01 & $25.6 \times 25.6$ & $n / a$ & 1.0 & MWPC \\
\hline $\mathrm{C} 6(\mathrm{x}, \mathrm{y})$ & 2498.77 & $51.2 \times 25.6$ & $\mathrm{n} / \mathrm{a}$ & 2.0 & MWPC \\
\hline $\mathrm{C} 7(\mathrm{u}, \mathrm{v})$ & 3012.52 & $25.6 \times 25.6$ & $\mathrm{n} / \mathrm{a}$ & 2.0 & MWPC \\
\hline $\mathrm{C} 8(\mathrm{u}, \mathrm{v})$ & 3088.65 & $51.2 \times 51.2$ & $\mathrm{n} / \mathrm{a}$ & 2.0 & MWPC \\
\hline $\mathrm{C} 9(\mathrm{x}, \mathrm{y}, \mathrm{u})$ & 3697.06 & $51.2 \times 51.2$ & $\mathrm{n} / \mathrm{a}$ & $2.0(2.8)$ & MWPC \\
\hline $\mathrm{C} 10(\mathrm{x}, \mathrm{y})$ & 4261.18 & $63.8 \times 25.6$ & $\mathrm{n} / \mathrm{a}$ & 2.0 & MWPC \\
\hline $\mathrm{C} 11(\mathrm{x}, \mathrm{y})$ & 4840.40 & $128.0 \times 38.4$ & $\mathrm{n} / \mathrm{a}$ & 2.0 & MWPC \\
\hline $\mathrm{C} 12(\mathrm{x}, \mathrm{y})$ & 6154.35 & $128.0 \times 38.4$ & $\mathrm{n} / \mathrm{a}$ & 2.0 & MWPC \\
\hline
\end{tabular}

Table 2.2: The z-positions and size of the detectors in the E 800 spectrometer.

\subsubsection{The Silicon Strip Detectors (SSDs)}

The SSDs were positioned furthest upstream in the spectrometer to allow accurate tracking of the parent hyperon before it decayed. There were eight planes of silicon $300 \mu \mathrm{m}$ thick with 280 strips at $100 \mu \mathrm{m}$ pitch (these were manufactured by Hammamatsu). The SSDs were mounted in specially designed stands which allowed adjustment of the individual planes in the $\mathrm{x}$ and $\mathrm{y}$ directions and rotations about the $\mathrm{z}$-axis. The entire array could also be levelled and adjusted in the vertical direction. The SSD 
stands were bolted to a $0.47 \times 0.62 \times 1.7 \mathrm{~m}^{3}$ cement shielding block for stability.

Laben model MSP1 preamplifiers amplified the charge (on the order of $1 \mathrm{fC}$ ) collected on a strip when a particle passed through the detector, giving an output pulse of 1.0- $2.0 \mathrm{mV}$. The preamplifier output pulses were then amplified and discriminated by Nanometric N-277 amplifier cards, with discrimination thresholds set at $0.65 \mathrm{mV}$. The outputs of the amplifier cards were latched and read out by the Nanometric N281 CAMAC readout system. The efficiency of the individual SSD planes is given in Table 2.3 .

\subsubsection{The Multi-wire Proportional Chambers (MWPCs)}

The main detectors used in this experiment were multi-wire proportional chambers (MWPCs). The detectors used an ionizing gas of $99.88 \%$ argon, $0.12 \%$ freon. The gas was bubbled through Methylal at $0.7^{\circ} \mathrm{C}$ to quench sparking between the anode and cathode planes. The final gas mixture in the chambers was roughly $95 \%$ argon, $5 \%$ methylal, and $0.12 \%$ freon. The gas was fed to each chamber in parallel from the gas distribution manifold. Back pressure was provided by bubblers filled with mineral oil on the gas output of the chambers. The bubblers also allowed visual confirmation that gas was actually flowing through the chambers.

The cathode planes of the chambers were kept at negative high voltage, which provided the proper electric field for ionization. The high voltage settings and efficiencies of the MWPCs are shown in Table 2.3. The optimal operating voltage for each chamber was determined by plotting a curve of relative efficiency against operating voltage as shown in Figure 2.7. The chambers where constructed with cathode planes "sandwiched" on either side of a sense plane. Most of these chambers had sense wires oriented in the $\mathrm{x}$ and $\mathrm{y}$ directions. Chambers $\mathrm{C} 7$, and $\mathrm{C} 8$ were rotated 45 degrees about the z-axis, which eliminated stereoscopic ambiguities. These rotated planes are known as " $u$ " and "v" planes. U planes gave $(x+y)$ information and v planes were (x-y). C9 had 
a 11-plane in addition to the standard $x$ and $y$ planes this plane had a pitch of $2 \sqrt{2} \mathrm{~mm}$ and operated with a high voltage offset of $300 \mathrm{~V}$ to give a gas gain similar to the $2 \mathrm{~mm}$ pitch planes.

\begin{tabular}{|c|c|c|c|c|c|}
\hline Detector & Voltage & Efficiency & Detector & Voltage & Efficiency \\
\hline S1 & $2.15 \mathrm{kV}$ & 0.98 & S2 & $1.60 \mathrm{kV}$ & 0.97 \\
\hline $\mathrm{V} 1 \mathrm{E}$ & $1.80 \mathrm{kV}$ & 0.97 & $\mathrm{~V} 2 \mathrm{E}$ & $2.15 \mathrm{kV}$ & 0.96 \\
\hline $\mathrm{V} 1 \mathrm{~W}$ & $1.70 \mathrm{kV}$ & 0.98 & $\mathrm{~V} 2 \mathrm{~W}$ & $2.15 \mathrm{kV}$ & 0.98 \\
\hline $\mathrm{SSD} 1 \mathrm{x}$ & $36 \mathrm{~V}$ & 0.83 & $\mathrm{SSD} 2 \mathrm{y}$ & $36 \mathrm{~V}$ & 0.63 \\
\hline $\mathrm{SSD} 3 \mathrm{x}$ & $36 \mathrm{~V}$ & 0.83 & $\mathrm{SSD} 4 \mathrm{y}$ & $36 \mathrm{~V}$ & 0.84 \\
\hline $\mathrm{SSD} 5 \mathrm{x}$ & $36 \mathrm{~V}$ & 0.81 & $\mathrm{SSD} 6 \mathrm{y}$ & $36 \mathrm{~V}$ & 0.85 \\
\hline $\mathrm{SSD} 7 \mathrm{x}$ & $36 \mathrm{~V}$ & 0.84 & $\mathrm{SSD} 8 \mathrm{y}$ & $36 \mathrm{~V}$ & 0.82 \\
\hline $\mathrm{C} 1 \mathrm{x} / \mathrm{y}$ & $2.66 \mathrm{kV}$ & $0.94 / 0.93$ & $\mathrm{C} 2 \mathrm{x} / \mathrm{y}$ & $2.66 \mathrm{kV}$ & $0.92 / 0.94$ \\
\hline $\mathrm{C} 3 \mathrm{x} / \mathrm{y}$ & $2.66 \mathrm{kV}$ & $0.95 / 0.95$ & $\mathrm{C} 4 \mathrm{x} / \mathrm{y}$ & $3.82 \mathrm{kV}$ & $0.69 / 0.67$ \\
\hline $\mathrm{C} 5 \mathrm{x} / \mathrm{y}$ & $3.83 \mathrm{kV}$ & $0.93 / 0.94$ & $\mathrm{C} 6 \mathrm{x} / \mathrm{y}$ & $2.94 \mathrm{kV}$ & $0.98 / 0.99$ \\
\hline $\mathrm{C} 7 \mathrm{u} / \mathrm{v}$ & $2.85 \mathrm{kV}$ & $0.94 / 0.98$ & $\mathrm{C} 8 \mathrm{u} / \mathrm{v}$ & $2.80 \mathrm{kV}$ & $0.96 / 0.98$ \\
\hline $\mathrm{C} 9 \mathrm{x} / \mathrm{y} / \mathrm{u}$ & $2.91 \mathrm{kV} / 0.45 \mathrm{kV}$ & $0.96 / 0.98 / 0.96$ & $\mathrm{C} 10 \mathrm{x} / \mathrm{y}$ & $3.02 \mathrm{kV}$ & $0.98 / 1.00$ \\
\hline $\mathrm{C} 11 \mathrm{x} / \mathrm{y}$ & $3.03 \mathrm{kV}$ & $0.95 / 0.97$ & $\mathrm{C} 12 \mathrm{x} / \mathrm{y}$ & $3.15 \mathrm{kV}$ & $0.96 / 0.97$ \\
\hline
\end{tabular}

Table 2.3: The voltages and efficiencies of the detectors.

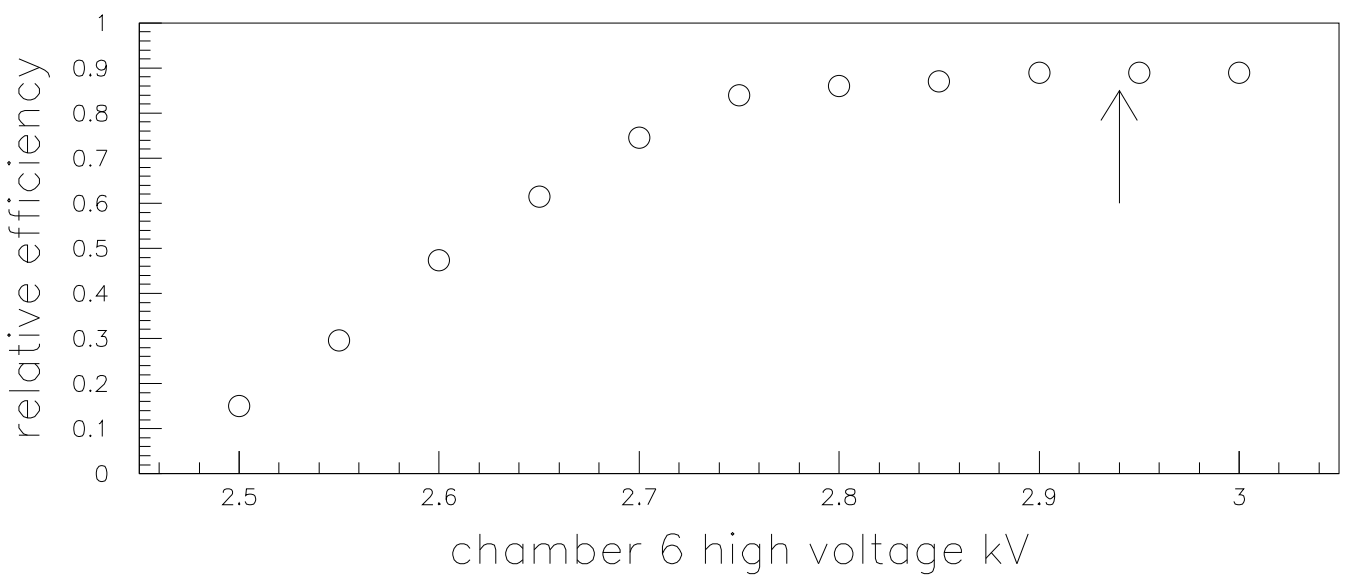

Figure 2.7: The plateau curve for chamber 6 . The vertical axis is the relative efficiency and the horizonal axis is the high voltage in $\mathrm{kV}$. The arrow indicates the operating voltage of the chamber. 
The three most upstream chambers used in this experiment $\mathrm{C} 1, \mathrm{C} 2$, and $\mathrm{C} 3$ were $1 \mathrm{~mm}$ wire spacing MWPCs manufactured by Fermilab. These chambers used 12820 micron gold-plated tungsten wires in both the $\mathrm{x}$ and $\mathrm{y}$ cathodes, and $0.127 \mathrm{~mm}$ thick aluminum foil anodes. The signals from the wires of these chambers were amplified and discriminated by Lecroy model 277-CD amplifier cards. The signals from the amplifiers were latched and read out by the same Nanometric N-281 CAMAC system as the SSDs used.

The rest of the chambers were constructed for previous experiments for the Fermilab hyperon group $[3,4,5,6,8]$. Chambers $\mathrm{C} 4$ and $\mathrm{C} 5$ were large $1 \mathrm{~mm}$ chambers using 20 micron gold-plated tungsten wire for the cathode planes. C4 used 25 micron copperplated beryllium wire at $0.5 \mathrm{~mm}$ pitch for the anodes, while $\mathrm{C} 5$ used $0.127 \mathrm{~mm}$ aluminum foil anode planes. The rest of the planes used, with the exception of $\mathrm{C} 9$, consisted of $2 \mathrm{~mm}$ pitch anode planes of 25 micron gold-plated tungsten wire and cathode planes of $1 \mathrm{~mm}$ pitch of 50 micron copper-beryllium wires. As stated above C9 had an additional u-plane, which had $2.8 \mathrm{~mm}$ pitch and was constructed with 20 micron gold-plated tungsten wire cathodes. The anodes used in C9 were 75 micron copper-beryllium wire.

The above chambers, C4-C12, used a custom built CAMAC driven readout system. The signals from the chamber wires were fed directly into chamber mounted amplifier-discriminator-latch cards. The discrimination level for the input pulse was approximately $2 \mathrm{mV}$ for $\mathrm{C} 5-\mathrm{C} 9$ and $1 \mathrm{mV}$ for $\mathrm{C} 10-\mathrm{C} 12$. [44] To allow adequate time for triggering, the latches used a one-shot delay of 750 nanoseconds. The latches in the chambers formed a sequential chain, starting with the most downstream chamber, C12. The system was readout using a custom built CAMAC interface module. [44]

\subsubsection{The Analysis Magnets}

Two BM109 dipole magnets with vertical fields were used to measure the total momenta of the final decay products. These magnets were $182 \mathrm{~cm}$ in length with pole pieces 
$170.2 \mathrm{~cm}$ in length. The upstream magnet's pole piece was located from $3786.45 \mathrm{~cm}$ to $3956.65 \mathrm{~cm}$ downstream of the downstream face of the precession magnet. The second magnet's pole piece was located $4025.70 \mathrm{~cm}$ to $4195.9 \mathrm{~cm}$ downstream of the precession magnet face. The magnets were centered in $\mathrm{x}$ and $\mathrm{y}$ on the $\mathrm{z}$-axis of the experiment. The magnets had an $x$ aperture of $61.0 \mathrm{~cm}$. The upstream magnet had a y aperture of $25.4 \mathrm{~cm}$, the downstream magnet's y aperture was $30.5 \mathrm{~cm}$. The BM109s used $10 \mathrm{~cm}$ thick iron mirror plates located $30 \mathrm{~cm}$ from the ends of the pole pieces to reduce the fringe fields produced by these magnets.

The fields of these magnets were measured using two different techniques. The first method, known as "Ziptrack II", measured the field strength of the magnets at points in a three dimensional lattice within the apertures of the magnets. This method allowed calculation of the field integral for several $\mathrm{x}$ and $\mathrm{y}$ positions in the magnets. During the experiment we found it necessary to ramp the magnet current. Since the measurements had to be made with a steady current, we tested the consistency of ramped operation versus steady state operation using isolated "Ziptrack II" measurements at selected positions. The tests confirmed that the difference between the two modes was less than $0.08 \%$ and yielded a combined field integral for the two magnets of $-4.817 \mathrm{Tm}$. The thin lens approximation for this field integral gives an effective transverse momentum, the "p $\mathrm{p}_{T}$ kick", of $-1.445 \pm 0.03 \mathrm{GeV} / \mathrm{c}$.

The second technique for determining the total field integral of the magnets was to simultaneously fit both the $\Lambda$ and $\Xi^{-}$masses using the reconstruction program. By this method the $\mathrm{p}_{T}$ kick was found to be $-1.465 \pm 0.02 \mathrm{Gev} / \mathrm{c}$, in good agreement with the field measurement. The final measurement used a $\mathrm{p}_{T}$ kick value of $-1.465 \mathrm{GeV} / \mathrm{c}$. 


\subsection{The Trigger}

The E800 trigger selected events where the charged particles matched the pattern of a topology of a charged particle decaying to two negatively and one positively charged particle. In addition to the $\Xi^{-}$'s and $\Omega^{-}$'s the beam through the E800 spectrometer contained $\pi^{-} \mathrm{s}, \mathrm{K}^{-} \mathrm{s}, \bar{p} \mathrm{~s}$, es, $\mu^{-} \mathrm{s}$, and $\bar{\Sigma}^{-}$created at the second target, as well as particles created in the spectrometer itself through the interaction of beam particles with material in the beamline.

Two triggers were used during data gathering. The first was a loose trigger which was primarily "one-track" events. It required a coincidence of the $10 \mathrm{~ns}$ wide signal from the beam counter, $\mathrm{S} 2$, with a delayed $10 \mathrm{~ns}$ wide signal from the upstream beam counter, S1. Two sets of veto counters, V1 and V2, were placed just outside the experimental aperture, which reduced events from beamline interactions. A signal in any of the veto counters would negate the trigger. This set of requirements gave the one track trigger

$$
\text { one }-\operatorname{track}=S 1 \cdot S 2 \cdot \overline{V 1} \cdot \overline{V 2}
$$

Events satisfying this trigger were prescaled by factors between 1028 and 4096 during data taking before they were written to tape. Data satisfying this trigger were used to align the experiment and study the efficiencies of the detectors.

In order to isolate the $\Xi^{-}$and $\Omega^{-}$candidates a second "three-track" trigger was devised. This trigger was based on the topology of the desired decay chains, $\Omega^{-} \rightarrow \Lambda \mathrm{K}^{-}$. $\Lambda \rightarrow \mathrm{p} \pi^{-}\left(\Xi^{-} \rightarrow \Lambda \pi^{-}, \Lambda \rightarrow \mathrm{p} \pi^{-}\right)$. These decays culminate in two low momentum negatively charged particles $\left(\mathrm{K}^{-}, \pi^{-}\right)$and a single high momentum positively charged particle $(p)$. A combination of the one-track trigger and a hit in the negative particle region of $\mathrm{C} 11$, "C11 $\pi^{-}$", and a hit in the positive particle region of $\mathrm{C} 12$, "C12 proton" give a sufficiently selective trigger. The positions of these regions were initially determined from monte carlo simulations of the experiment. The final positions were determined 
from calibration data.

$$
\text { three }- \text { track }=S 1 \cdot S 2 \cdot \overline{V 1} \cdot \overline{V 2} \cdot C 11_{\pi} \cdot C 12_{\text {proton }}
$$

The signals from trigger elements were 10ns in duration. This gave the trigger a high level of selectivity. Table 2.4 shows the rates at several points in the trigger system. This demonstrates the reduction of spurious events included in the sample by the use of the one-track and three-track triggers.

\begin{tabular}{|c|c|c|c|}
\hline Detector or & \multicolumn{3}{|c|}{ Rates for detectors and triggers in $\mathrm{kHz}$} \\
\hline Trigger & Omr neutral & $+1.8 \mathrm{mr}$ neutral & $+1.8 \mathrm{mr}$ spin-transfer \\
\hline Protons & $1.95 \times 10^{1} 1$ & $1.74 \times 10^{1} 2$ & $2.52 \times 10^{1} 2$ \\
\hline Livetime & $58 \%$ & $61 \%$ & $79 \%$ \\
\hline$\overline{\mathrm{S} 1}$ & 59.0 & 184.5 & 177.8 \\
\hline $\mathrm{S} 2$ & 61.9 & 181.0 & 149.1 \\
\hline $\mathrm{S} 1 \cdot \mathrm{S} 2$ & 39.5 & 62.3 & 18.3 \\
\hline V1 & 176.9 & 385.9 & 418.2 \\
\hline $\mathrm{V} 2$ & 57.1 & 287.5 & 394.8 \\
\hline $\mathrm{V} 1+\mathrm{V} 2$ & 210.1 & 597.0 & 787.0 \\
\hline one-track & 28.9 & 51.0 & 11.3 \\
\hline $\mathrm{C} 11_{\pi^{-}}$ & 60.1 & 213.5 & 327.3 \\
\hline $\mathrm{C} 12_{\text {proton }}$ & 41.8 & 89.4 & 104.0 \\
\hline three-track & 1.27 & 1.63 & 0.85 \\
\hline C6 & 116.7 & 466.8 & 501.1 \\
\hline $\mathrm{C} 7$ & 70.2 & 198.7 & 186.9 \\
\hline C9 & 137.9 & 475.4 & 562.3 \\
\hline
\end{tabular}

Table 2.4: The singles rates for various detectors and triggers for three production modes at typical running intensities.

\subsection{The Data Aquisition System}

\subsubsection{Operational Overview}

The E800 data acquisition system was designed to minimize computer generated deadtime and to monitor data quality as the experiment ran. This was accomplished by gathering the data during the 20 second spill-on period, and logging to tape during the 
40 second spill-off portion of the accelerator cycle.

During the spill-on time, when a good trigger was detected, the smart crate controller (SCC) would read out the detectors. The readout started with the $2 \mathrm{~mm} \mathrm{MWPCs,} \mathrm{and}$ continued to the $1 \mathrm{~mm}$ MWPCs and SSDs, and then the trigger latches were read into the SCC. After reading the data from the detectors, the SCC wrote the data to the first-in-first-out (FIFO) buffer. Finally the detectors were reset and reenabled. This cycle was repeated for each good trigger. The readout time for a typical event was approximately $300 \mu \mathrm{s}$. A typical spill yielded 20,000 to 30,000 triggers with a livetime of about $60 \%$.

During the spill-off period the data was transferred from the memory module FIFO via the custom VME-CAMAC interface to the Vax 3200. Fermilab Event Builder software would then transfer the data to the event-pool. Once in the event-pool the data was logged to tape and some fraction of the data was analyzed using Fermilab Buffer Manager software.

For logging purposes the data were divided into runs. Each run contained about 500,000 events. During data taking, a run was collected in 18-30 minutes. Typically ten runs were written to a single $8 \mathrm{~mm}$ tape. Table 2.5 shows the number of triggers taken under various run conditions.

\subsubsection{Software}

The software used for data aquisition was a subset of the Fermilab Vaxonline system. This package was made available through the Fermilab computing department data aquisition group. The system has programs which make the working environment easier, and programs that actually control the flow of data.

The environmental programs consisted of Global Menu, Courier, and Run Control. "Global Menu" is an interface allowing control of several data aquisition programs from a single menu driven window. "Courier" allows status messages to be routed over 


\begin{tabular}{c|c|c|c|c}
$\begin{array}{c}\text { Production } \\
\text { Mode }\end{array}$ & $\begin{array}{c}\text { Angle } \\
(\mathrm{mrad})\end{array}$ & $\begin{array}{c}\text { Current } \\
\text { (amps) }\end{array}$ & Runs & Triggers \\
\hline Neutral & -0.0 & -2900 & 172 & 84187513 \\
\hline Neutral & 0.0 & -2900 & 170 & 82531343 \\
\hline Neutral & -1.8 & -2900 & 757 & 374297889 \\
\hline Neutral & -1.8 & -750 & 289 & 142700032 \\
\hline Neutral & 1.8 & -2900 & 711 & 346982603 \\
\hline Neutral & 1.8 & -750 & 308 & 150467562 \\
\hline Spin Transfer & -1.8 & -2900 & 171 & 84086479 \\
\hline Spin Transfer & 1.8 & -2900 & 182 & 87777817 \\
\hline
\end{tabular}

Table 2.5: The number of runs and three-track triggers taken under various run conditions.

Decnet $^{T M}$ from the data aquisition computer to another Workstation. "Run Control" coordinates the data acquisition system. It passes data to the programs which do the hardware control and data logging.

The data acquisition was done using two of the programs, Event Builder and Output. "Event Builder" controlled the link between the hardware and the data acquisition computer. The incoming data were read in by Event Builder and placed in the event pool, a place in memory accessible to other programs. "Output" took the data from the event pool and wrote them to tape. An ancillary program, Buffer Manager, allowed access to the event pool by another Decnet node. By using Buffer Manager our experiment was able to analyze a fraction of the data online, and perform diagnostic tests of experimental hardware and software.

\subsubsection{Hardware}

The hardware for data acquisition was driven by a $\operatorname{Vax}^{T M} 3200$ with two $8 \mathrm{~mm}$ tape drives. The $\operatorname{Vax}^{T M}$ was interfaced to the CAMAC via a Jorway 411. The SCC gathered the data from the CAMAC data bus. As the data was gathered from CAMAC, the SCC took control of the CAMAC bus and put the data into a 16 Mbyte VME FIFO 
memory module, which could be read out by the $\operatorname{Vax}^{T} M$ via a specially built CAMAC to VME interface to the Jorway interface.

Several other Digital Equipment Corporation ${ }^{T M} \operatorname{Vax}^{T M}$ station model 3100s were installed in the remote counting room. The first of these Vax ${ }^{T M}$ stations was used to control data taking by the model 3200 station in another location. Another 3100 was used for online analysis of a fraction, typically $10 \%$, of the data as it was taken. This allowed online rate analysis and detector diagnostics. A third node was used for off line analysis as a further check of data quality. 


\section{Chapter 3}

\section{Event Reconstruction and Data Selection}

\subsection{Introduction}

The three track topology of the decay $\Omega^{-} \rightarrow \Lambda \mathrm{K}^{-}, \Lambda \rightarrow \mathrm{p} \pi^{-}$and the similar three track decay $\Xi^{-} \rightarrow \Lambda \pi^{-}, \Lambda \rightarrow \mathrm{p} \pi^{-}$allow full event reconstruction with only the information from the hits in the MWPC's. The charged particles resulting from the decays were bent in the $x-z$ plane by the momentum analyzing magnets. The particles charge was determined by the direction of bend in the analysis magnets. After the particle tracks were determined by geometric fit to the hits in the MWPCs the track momenta could be computed from the bend angles, and the opening angles of the decays could be calculated. This information was used to calculate the parent mass under either the $\Omega^{-}$or $\Xi^{-}$decay hypothesis,

$$
m_{\Lambda}^{2}=m_{\pi}^{2}+m_{\text {proton }}^{2}+2 E_{\pi} E_{\text {proton }}-2 \vec{P}_{\pi} \cdot \vec{P}_{\text {proton }}
$$

and 


$$
m_{\Xi, \Omega}^{2}=m_{\pi, K}^{2}+m_{\Lambda}^{2}+2 E_{\pi, K} E_{\Lambda}-2 \vec{P}_{\pi, K} \cdot \vec{P}_{\Lambda}
$$

where the $\vec{P}$ and E are the particles' three-vector momenta and energies and the $\mathrm{m}_{x}$ are the rest masses. The computation of the parent mass is made under the assumption of the daughter particle identities.

\subsection{The First Pass}

Our initial pass through the raw data tapes eliminated events that lacked enough information to be reconstructed as three track events. The data selection criteria used for this was a simple hit count in the chambers. Table 3.1 shows the percentage of three track triggers eliminated by this selection criteria.

\begin{tabular}{l|c} 
Criteria Description & Percentage of Triggers Eliminated \\
\hline more than 2 hits in each of 3 planes of 6,8 & 62.5 \\
more than 3 hits in each plane of 7,8 & 0.0 \\
and more than 2 hits in the 9 uplane & 2.8 \\
more than 2 hits in 2 x-planes of $10,11,12$ & 5.5 \\
more than 2 hits in 3 y-planes of $6,7,8,9,10,11,12$ &
\end{tabular}

Table 3.1: The first pass

The events which passed these criteria were written to the first level data summary or " $\mathrm{ds} 0$ " tapes. Table 3.2 gives the number of ds0 file candidates for each beam configuration in the data sample.

Good single track events, which had two $\mathrm{x}$ hits on the upstream and downstream $\mathrm{x}$ views and three hits in the y view, were written to a separate set of tapes to be used for calibration, and alignment of the spectrometer, as well as possible single track analysis. 


\begin{tabular}{|c|c|c|c|c|c|}
\hline $\begin{array}{c}\text { Production } \\
\text { Mode }\end{array}$ & $\begin{array}{c}\text { Angle } \\
(\mathrm{mrad})\end{array}$ & $\begin{array}{c}\text { Current } \\
(\mathrm{amps})\end{array}$ & Runs & Triggers & $\begin{array}{c}\text { Three Track } \\
\text { Candidates }\end{array}$ \\
\hline Neutral & -0.0 & -2900 & 172 & 84187513 & 39097574 \\
\hline Neutral & 0.0 & -2900 & 170 & 82531343 & 38000350 \\
\hline Neutral & -1.8 & -2900 & 757 & 374297889 & 110232099 \\
\hline Neutral & -1.8 & -750 & 289 & 142700032 & 45346166 \\
\hline Neutral & 1.8 & -2900 & 711 & 346982603 & 102177861 \\
\hline Neutral & 1.8 & -750 & 308 & 150467562 & 46929723 \\
\hline Spin Transfer & -1.8 & -2900 & 171 & 84086479 & 30240120 \\
\hline Spin Transfer & 1.8 & -2900 & 182 & 87777817 & 34212605 \\
\hline
\end{tabular}

Table 3.2: The first pass analysis event totals.

\subsection{Full Reconstruction}

E800 used three reconstruction strategies to efficiently reconstruct the three track events. The different reconstruction strategies were arranged such that the fastest method processed all of the events and the other strategies processed only those events not well determined by the first strategy. The successive passes were each more sophisticated, and each recovered fewer events, which gave us high reconstruction efficiency with minimal computing time.

The reconstruction program is shown schematically in Figure 3.1.

The first step of the procedure applied more hit counting criteria to remove events without enough information to be unambiguously reconstructed. These criteria were:

- more than 2 hits in each X-plane of C7, C9, C10, and C11

- more than 2 hits in each y-plane of $\mathrm{C} 7, \mathrm{C} 9$, and $\mathrm{C} 10$

Events meeting the above criteria were passed to the reconstruction routines.

The first pass of the reconstruction correlates the hits into straight tracks and connects the $\mathrm{x}$ and $\mathrm{y}$ tracks through the $1 \mathrm{and} \mathrm{v}$ view planes. Tracks were fit using $\chi^{2}$ minimization to make the best hit assignments. The possible tracks were assigned 


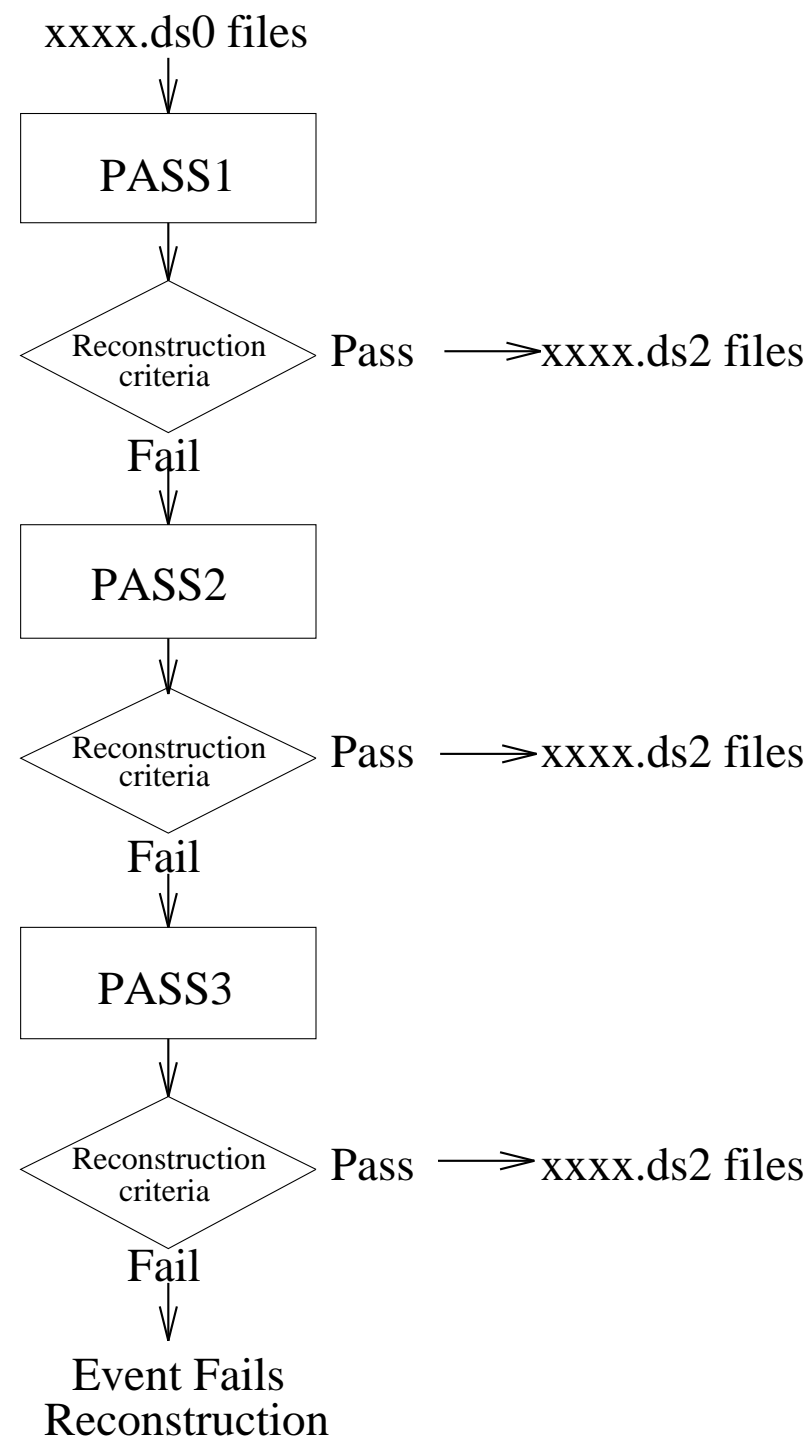

Figure 3.1: Schematic diagram of the E800 reconstruction program 
momenta and charges. The proton was assumed to be on the positive track with the smallest bend angle and the other tracks were assigned to make the best $\Lambda$ mass and vertex. The remaining track was assumed to be the $\mathrm{K}^{-}\left(\pi^{-}\right)$from the $\Omega^{-}\left(\Xi^{-}\right)$decay. The first pass (PASS1) was very fast (0.02s/event) and correctly reconstructed $67.5 \%$ of .ds0 level events. The events which failed this level of the reconstruction were characterized by missing hit information, or an excess of hits in the chambers due to noise, or by tracks which were too close to each other in the $\mathrm{x}$ or $\mathrm{y}$ views.

We used our monte carlo simulation to study events which PASS1 reconstructed and found a set of criteria we could use to select well reconstructed events for further analysis. These were:

- Geometric $\chi_{G}^{2} /$ degree of freedom $<4.0$

- Kinematic $\chi_{K}^{2}<15.0$

- Reconstructed $\Omega^{-}\left(\Xi^{-}\right)$mass within $15.0 \mathrm{MeV}$ of known mass (for either mass hypothesis)

Where geometric $\chi^{2}, \chi_{G}^{2}$ is the $\chi^{2}$ of the straight line fits for all the tracks in both the $\mathrm{x}$ and $\mathrm{y}$ views constrained to the two vertex three track topology. The "kinematic" $\chi^{2}$, $\chi_{K}^{2}$ is a figure of merit for the reconstruction of the $\Lambda$ which compares the reconstructed $\Lambda$ mass with the known $\Lambda$ mass.

Events reconstructed by PASS1 but failing these criteria were passed to the second stage of the reconstruction, PASS2. This reconstruction attempted to make all possible sets of tracks through the spectrometer. The tracks were constructed by starting with all sets of two hits in the upstream $\mathrm{x}$ and $\mathrm{y}$ views and adding hits to the track "stubs". Tracks from the upstream and downstream $x$ were joined at the analysis magnet bendplane and the $\mathrm{x}$ and $\mathrm{y}$ views were connected using the $u$ and $\mathrm{v}$ view information. PASS2 differed from the PASS1 reconstruction in that an event could have several possible solutions. These solutions were evaluated using $\chi_{G}^{2}$, the calculated $\Lambda$ mass, and either 
the $\Xi^{-}$or the $\Omega^{-}$mass. Using these quantities a single "best" solution was attained by ranking the solutions in order of $\chi_{G}^{2} /$ d.f.. All solutions with $\chi_{G}^{2}$ within 0.2 of the solution with the lowest $\chi_{G}^{2} /$ d.f. are then ranked using $\chi_{K}^{2}$. The remaining event with the lowest $\chi_{K}^{2}$ is the accepted solution. The selection criteria above were applied to the events again after this stage. Events passing the criteria were written to the candidate, “.ds2" files.

An event failing the criteria after PASS2 entered the third stage of the reconstruction, PASS3. This stage of the reconstruction attempted to separate the first daughter particle, the $\mathrm{K}^{-}$or $\pi^{-}$, from the $\Lambda$ decay products. After identifying several candidates for the upstream track of the $\mathrm{K}^{-} / \pi^{-}$from the SSD and upstream chamber hits the upstream daughter tracks were constructed through the rest of the spectrometer. The other possible tracks were fit to a $\Lambda$ decay topology. The solutions for the $\Lambda$ and the upstream daughter were combined and the parent mass was computed under both decay hypotheses $\left(\Omega^{-}, \Xi^{-}\right)$. After this stage the selection criteria were applied again. Events passing were written to files of either $\Xi^{-}$and/or $\Omega^{-}$candidates, events failing this pass were no longer kept in the data set.

Table 3.3 shows the percentage of data reconstructed by each pass of the reconstruction. The percentage of correctly reconstructed events was computed from monte-carlo simulated data reconstructed with known solutions. The total efficiency of the reconstruction for three track events constrained to decay within the decay region was $97 /$

\subsection{The Monte Carlo Simulation}

The E800 monte carlo simulation program generated events which simulated real data closely as possible. The program was used to design the experiment as well as to study the reconstruction and the data selection criteria used in the analysis. The monte carlo is not used in the actual analysis of the data. 


\begin{tabular}{l|c|c} 
Reconstruction Routine(s) & Percentage of Events to .ds2 & Percentage Correct \\
\hline Hit count criteria & 0.0 & - \\
PASS1 & 71.8 & 97.5 \\
PASS2 & 14.3 & 95.3 \\
PASS3 & 13.9 & 95.4 \\
Total & 100.0 & 96.9
\end{tabular}

Table 3.3: The percentage of .ds0 events reconstructed by various reconstruction routines. An event was reconstructed correctly when there were two or more correctly assigned hit for each track both upstream and downstream of the analysis magnet in the $\mathrm{x}$-view and three or more correctly assigned hits for each track in the $\mathrm{y}$-view.

Parent particles were created at the downstream target according to a distribution which was gaussian in $x$ any and had a exponential $z$ dependence. The parent particles were tracked through the collimator and allowed to decay using the appropriate lifetime given in the Particle Properties Data Book [36]. All unstable daughter particles from decays were also allowed to decay.

The charged particles were tracked through the spectrometer, and bent in the analysis magnets using the thin lens approximation by adding transverse momentum appropriate to each magnet, which was $-0.8084 \mathrm{GeV} / \mathrm{c}$ for the first magnet and -0.6559 $\mathrm{GeV} / \mathrm{c}$ for the second. The ratio of the fields came from the Ziptrack II measurements. Projecting the particles through the magnets using circular trajectories with appropriate fringe fields gave results identical to those using the computationally faster thin lens approximation.

The $\mathrm{x}$ and $\mathrm{y}$ position of the charged particles were calculated at each MWPC plane, SSD plane and at all scintillation counters. Using the chamber centers from the real data alignment files the positions of the particles were digitized to make wire hits. The positions at the trigger counters and $\mathrm{C} 11$ and $\mathrm{C} 12$ were used to check that the event satisfied the trigger. Events which satisfied the trigger were written to files in the same format as ".ds0" files which were read by the reconstruction program.

The above constituted "perfect" monte carlo, which includes the spectrometer resolution but no other effects. In reality the experiment was more complicated and many 
other details of the experiment were included in the monte carlo. Chamber inefficiencies including dead and hot wires were measured from data and simulated in the program. Beam associated noise rates were also investigated and fake noise was generated in a gaussian cross section as observed in the data. The momenta of the charged particles were altered slightly by small angle coulomb multiple scattering at each piece of material, and at wire planes. A list of all the material in the spectrometer was compiled with the thickness given in radiation lengths. The total amount of material in the experiment was $9.55 \times 10^{-2}$ radiation lengths. We also simulated the fringe field of the precession magnet. We modelled the fringe field as

$$
\vec{B}(z)_{\text {fringe }}=\frac{\beta}{\left(z_{0}-z\right)^{3}} \hat{y}
$$

where $\beta$ and $z_{0}$ are parameters determined from measurement of the field. We simulated the effect of this fringe field by integrating it and changing the particle momentum by the appropriate $\mathrm{p}_{T}$ kick at the point where the particle first encounters the field. The inclusion of the fringe field improved the mass comparison with data.

The largest improvement in the monte carlo program was to include field variations in the momentum analysis magnets. We found this effect in the data by plotting the $\Xi^{-}$ and $\Lambda$ masses for various particle locations at the bend plane of the analysis magnets. The variations were simulated in the monte carlo as a linear falloff in the field as the distance from the centerline of the magnet increases. We found the $\mathrm{x}$-variation was larger than the y variation. The variation is given by

$\Delta B_{y}=0.0003637(x-4.0)^{2}-0.005454(x-4.0)+0.0002857(y-4.0)^{2}-0.003286(y-4.0)$

where $\Delta \mathrm{B}_{y}$ is the variation in the magnetic field in the $\mathrm{y}$-direction, and $\mathrm{x}$ and $\mathrm{y}$ are the $\mathrm{x}$ and $\mathrm{y}$ positions of the particles at the bendplane the units are in Tesla and $\mathrm{cm}$. This model of the variation greatly improved the match between the reconstructed 
monte carlo and data masses but does not affect the results of the analysis. The mass comparisons are shown in Figures 3.9 and 3.10.

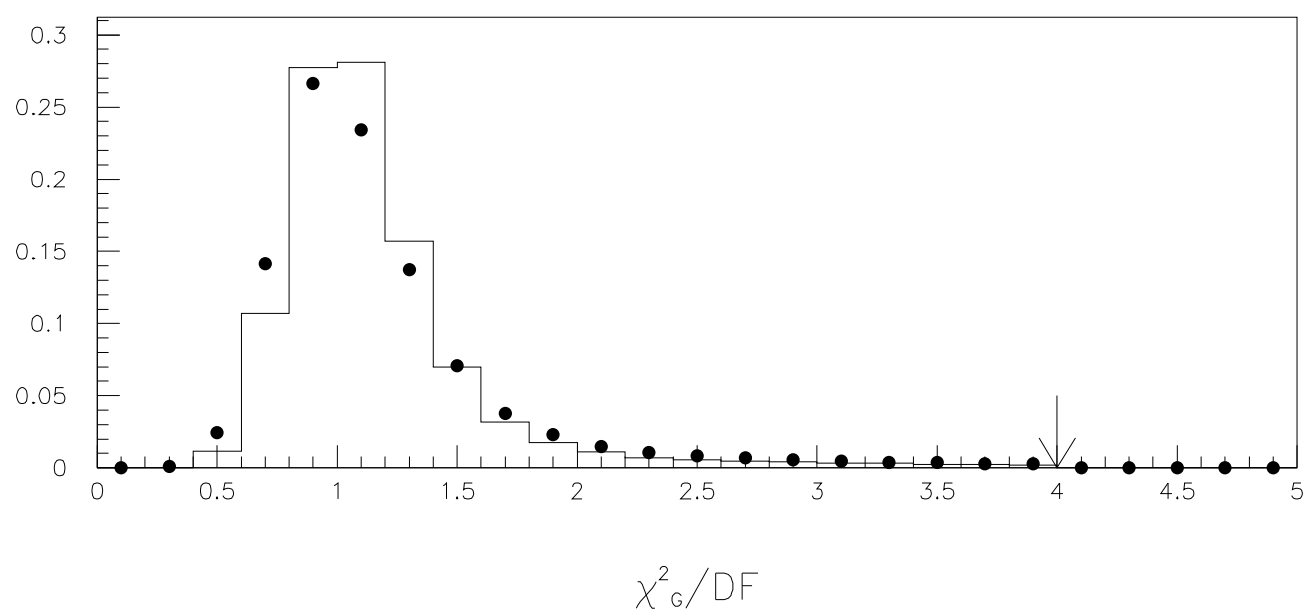

Figure 3.2: The geometric $\chi_{G}^{2}$ distribution for reconstructed data and monte carlo $\Omega^{-}$ events. The monte carlo data are shown with the solid lines and the data with dots. The arrow denotes the reconstruction selection criteria.

\subsection{Background and Data Selection Criteria}

Measuring the magnetic moment of the $\Omega^{-}$was complicated by the presence of background events in the data sample. The decay chains $\Omega^{-} \rightarrow \Lambda \mathrm{K}^{-}$and $\Xi^{-} \rightarrow \Lambda \pi^{-}$with $\Lambda \rightarrow \pi^{-} \mathrm{p}$ are very similar and the $\Xi^{-}$formed the most abundant background to the $\Omega^{-}$, since the ratio of $\Xi^{-}$to $\Omega^{-}$was $96: 1$. There were also other backgrounds to the $\Omega^{-}$sample. The decay $\Omega^{-} \rightarrow \Xi^{0} \pi^{-}$, with $\Xi^{0} \rightarrow \Lambda \pi^{0}$, and $\Lambda \rightarrow \pi^{-}$p could trigger the experiment. The branching ratio for this decay is $28 \%$ compared to $67 \%$ for the decay under measurement.

We studied ways to reduce these backgrounds by generating large samples of each 


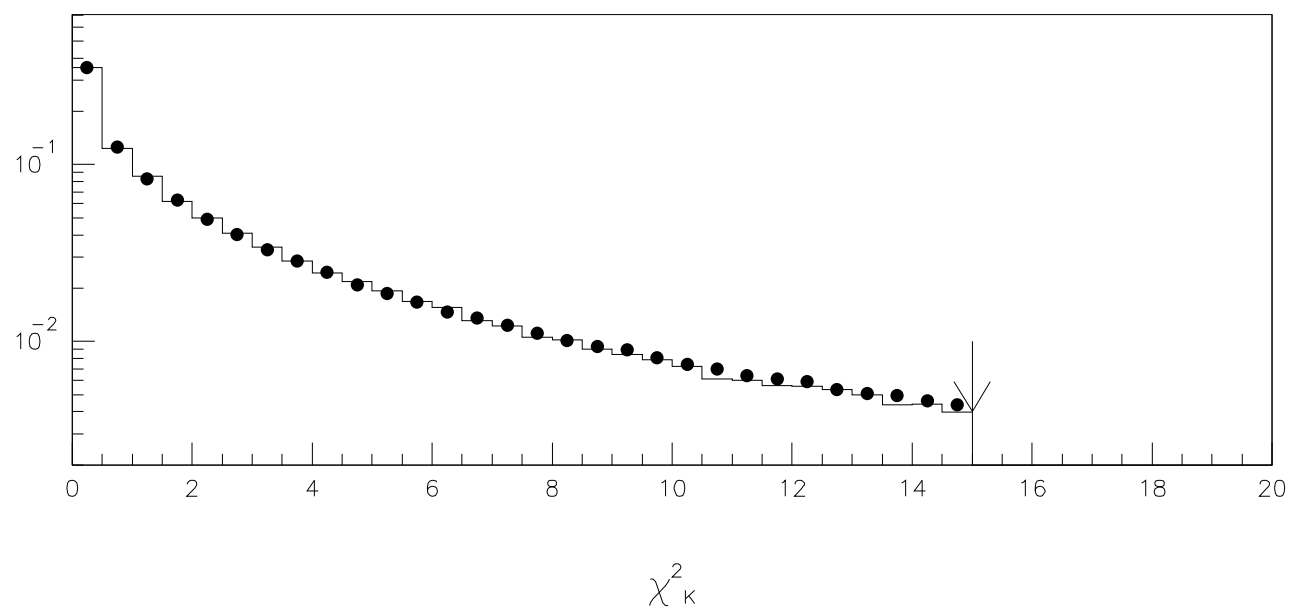

Figure 3.3: The kinematic $\chi_{K}^{2}$ distribution for reconstructed data and monte carlo $\Omega^{-}$ events. The monte carlo data are shown with the solid lines and the data with dots. The arrow denotes the reconstruction selection criteria.

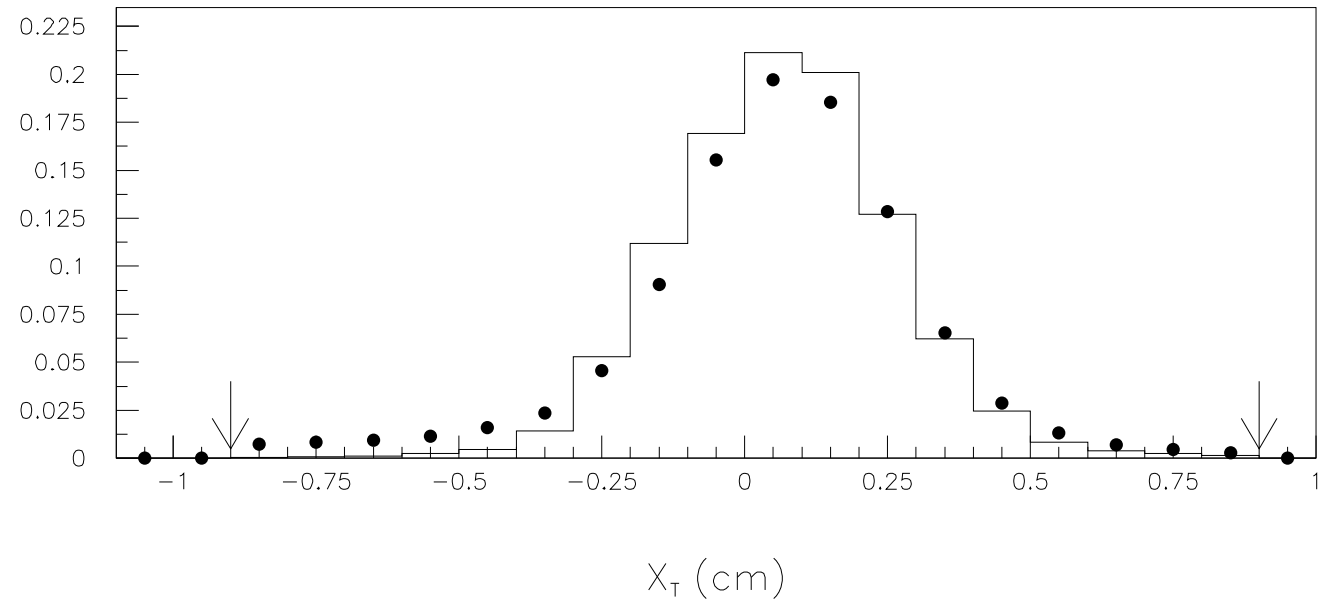

Figure 3.4: $\mathrm{X}_{T}$ (position in $\mathrm{x}$ in $\mathrm{cm}$. of the $\Omega^{-}$at the target) distribution for reconstructed data and monte carlo $\Omega^{-}$events. The monte carlo data are shown with the solid lines and the data with dots. The two arrows denote the reconstruction selection criteria. 


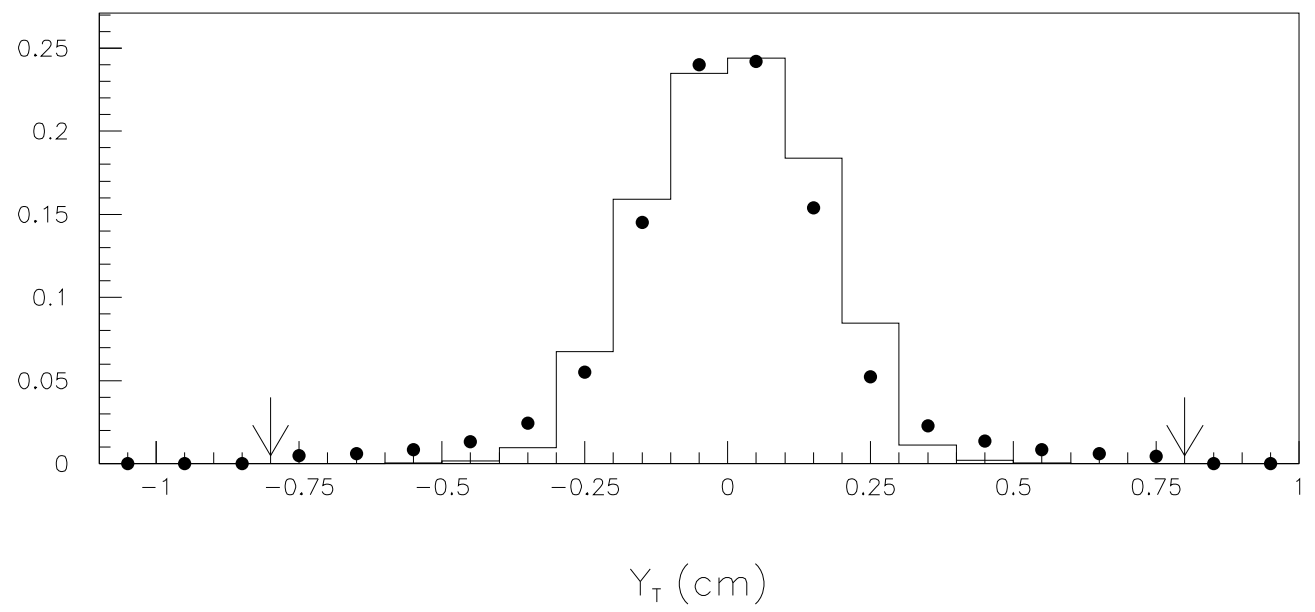

Figure 3.5: $\mathrm{Y}_{T}$ (position in $\mathrm{y}$ in $\mathrm{cm}$. of the $\Omega^{-}$at the target) distribution for reconstructed data and monte carlo $\Omega^{-}$events. The monte carlo data are shown with the solid lines and the data with dots. The two arrows denote the reconstruction selection criteria.

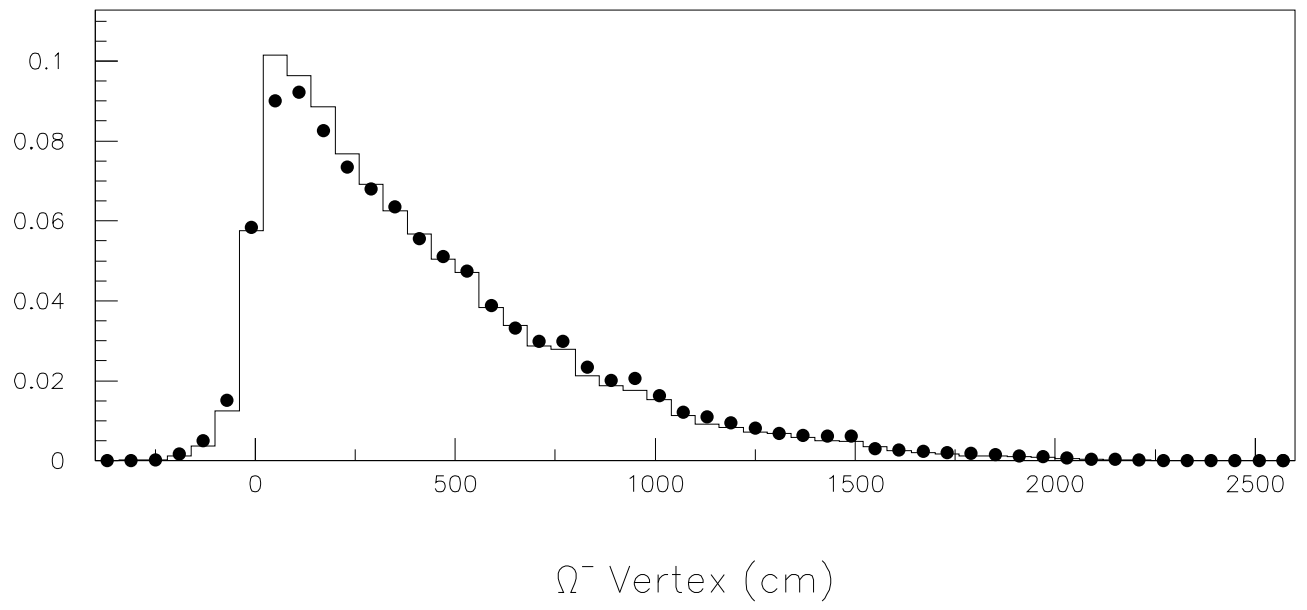

Figure 3.6: The $\Omega^{-}$vertex distribution for reconstructed data and monte carlo $\Omega^{-}$ events. The monte carlo data are shown with the solid lines and the data with dots 


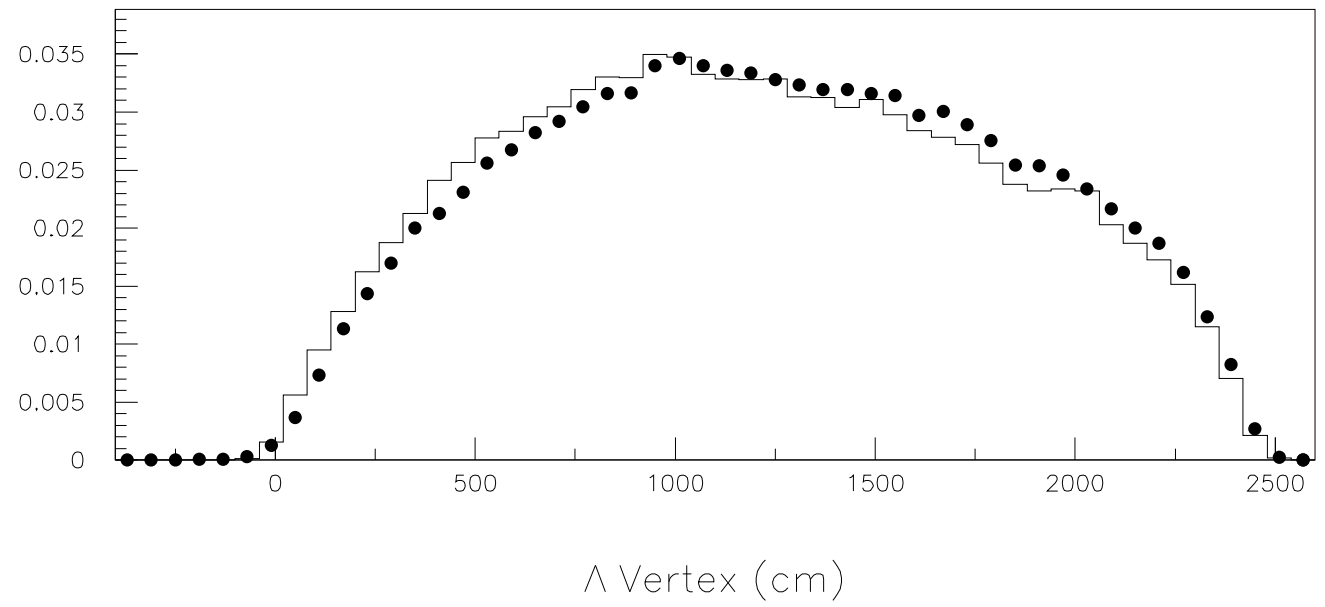

Figure 3.7: The $\Lambda$ vertex distribution for reconstructed data and monte carlo $\Omega^{-}$events. The monte carlo data are shown with the solid lines and the data with dots

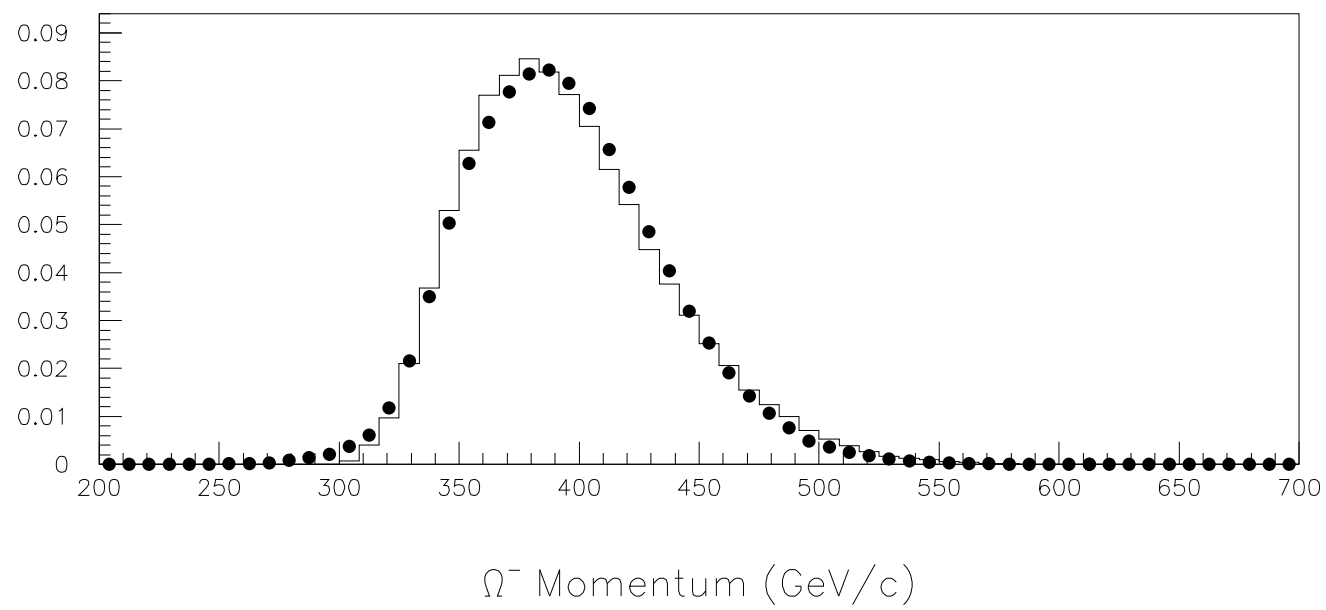

Figure 3.8: The total momentum distribution for reconstructed data and monte carlo $\Omega^{-}$events. The monte carlo data are shown with the solid lines and the data with dots. 


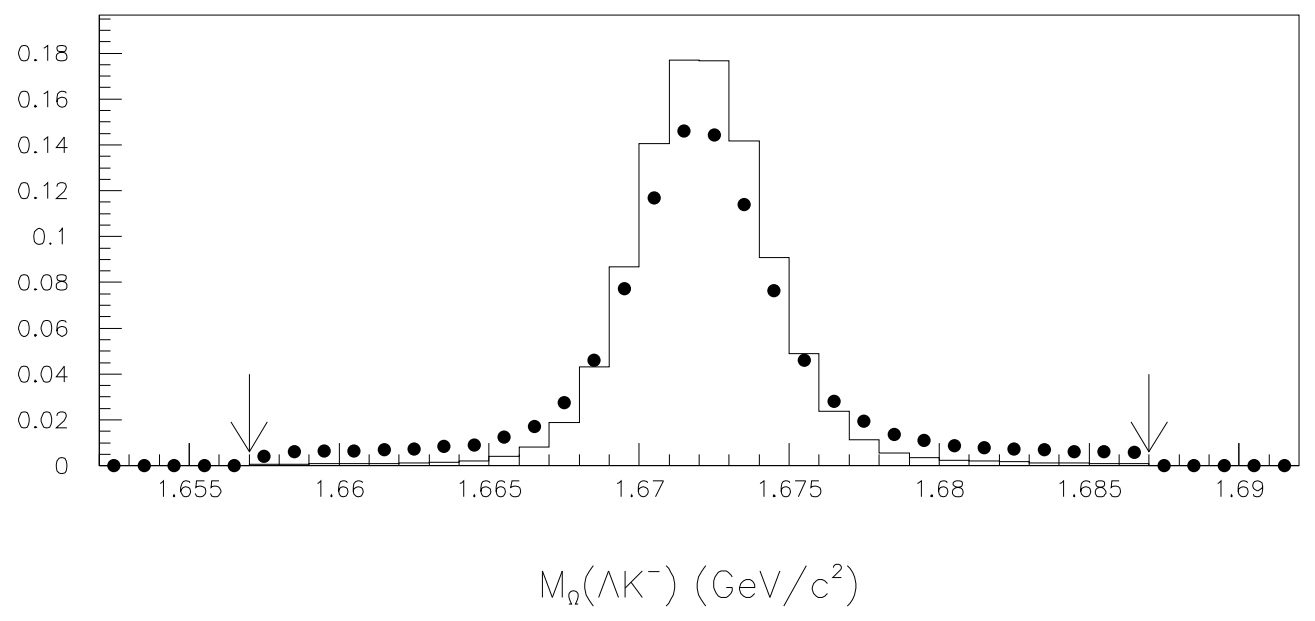

Figure 3.9: The $\Lambda$-K $\mathrm{K}^{-}$invariant mass distribution for reconstructed data and monte carlo $\Omega^{-}$events. The monte carlo data are shown with the solid lines and the data with dots. The two arrows denote the reconstruction selection criteria.

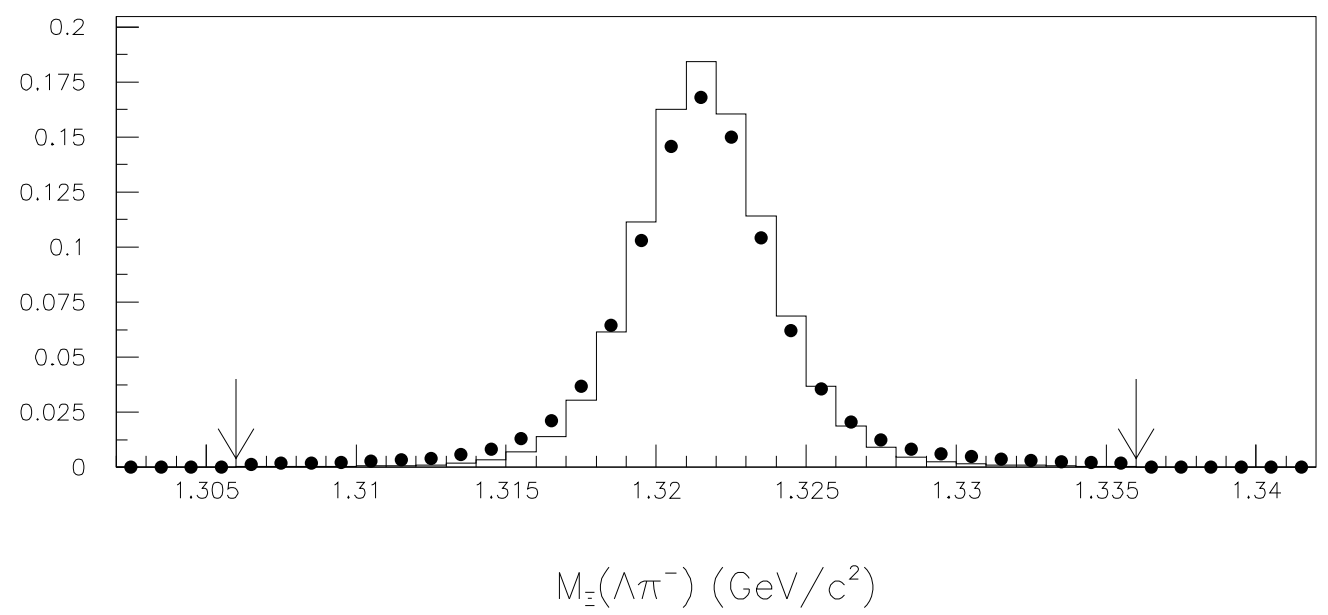

Figure 3.10: The $\Lambda$ - $\pi^{-}$invariant mass distribution for reconstructed data and monte carlo $\Xi^{-}$events. The monte carlo data are shown with the solid lines and the data with dots. The two arrows denote the reconstruction selection criteria. 
background using the monte carlo. The relative levels of the backgrounds were estimated from the trigger acceptance in the monte carlo, and the reconstruction acceptance for the background samples. We studied the effect of various combinations of selection criteria on the backgrounds using the reconstructed monte carlo samples.

Table 3.4 shows the selection criteria and their effects on the backgrounds.

\begin{tabular}{l|c|c} 
Acceptance of Events and & \multicolumn{2}{|c}{ Ratio of backgrounds(normalized to $\Omega^{-} \rightarrow \Lambda \mathrm{K}^{-}$) } \\
Data Selection Criteria & $\Xi^{-} \rightarrow \Lambda \pi^{-}$ & $\Omega^{-} \rightarrow \Xi^{0} \pi^{-}$ \\
\hline Accepted by trigger & 96.3 & 0.104 \\
Reconstructed in $\Omega^{-}$ds2 files & 10.5 & $0.0157 \pm 0.0002$ \\
Kinematic angle cut & $0.0030 \pm 0.0013$ & $0.0157 \pm 0.0002$ \\
Final relative sample content & $0.0030 \pm 0.0013$ & $0.0157 \pm 0.0002$
\end{tabular}

Table 3.4: The ratio of monte-carlo events for various backgrounds compared to $\Omega^{-} \rightarrow \Lambda$ $\mathrm{K}^{-}$events accepted and surviving data selection criteria.

Because the $\Xi^{-}$events fell in a well defined region of decay angles a kinematic angle cut was quite effective. If we plot the cosine of the angle of the $\mathrm{K}^{-}$in the parent particle rest frame under the $\Omega^{-}$hypothesis on the vertical axis and the azimuthal angle $\phi_{K}$ on the horizontal axis we find a well defined region containing the $\Xi^{-}$background events. Figures 3.11 and 3.12 show the distribution for reconstructed monte-carlo $\Omega^{-}$and $\Xi^{-}$ events. As one would expect the $\Omega^{-}$s are distributed evenly throughout the region, the $\Xi^{-} \mathrm{s}$ occupy only a small region of the $\cos \theta_{K}$ region. The third plot shows an actual data sample. Note that the number of $\Omega^{-}$is reduced in comparison to the number of $\Xi^{-}$. This is simply the $96: 1$ ratio of $\Xi^{-}$to $\Omega^{-}$events in the data. The next plot shows the $\cos \theta_{K} \phi$ plot for data after the $\Xi^{-}$region has been cut. The effect of the kinematic angle cut is illustrated in Figures 3.15 and 3.16, which show the $\Omega^{-}$and $\Xi^{-}$masses before and after the kinematic angle cut has been implemented.

The kinematic angle selection criteria reduced the $\Xi^{-} \rightarrow \Lambda \pi^{-}$background to the $0.25 \%$ level. However, there was still a significant amount of $\Omega^{-} \rightarrow \Xi^{0} \pi^{-}$events left in the sample.

Another way to estimate the background in the sample is to look at the tails of the 


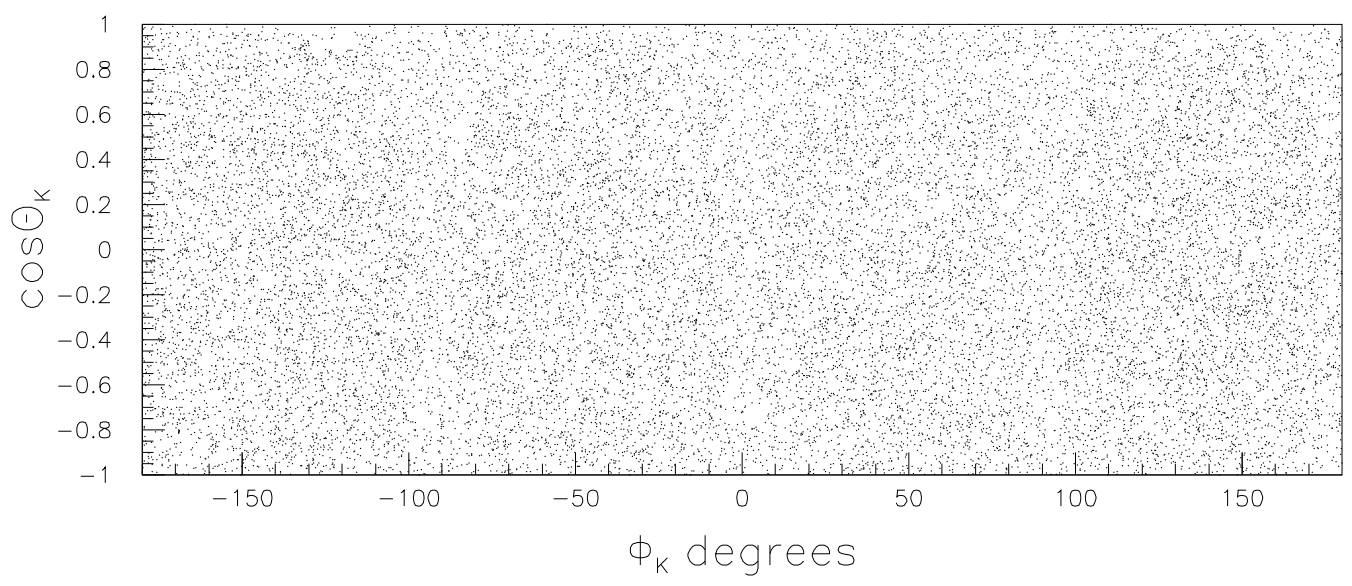

Figure 3.11: The $\cos \theta_{K}$ vs $\phi_{K}$ distribution for reconstructed monte-carlo $\Omega^{-}$events.

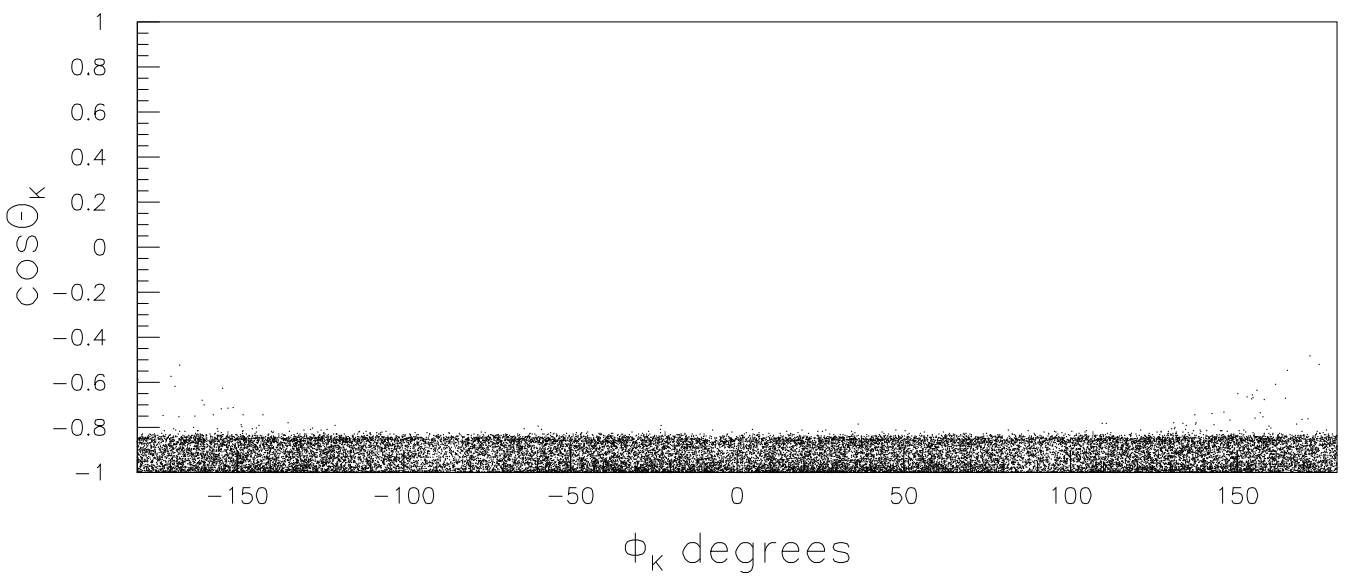

Figure 3.12: The $\cos \theta_{K}$ vs $\phi_{K}$ distribution for reconstructed monte-carlo $\Xi^{-}$events. 


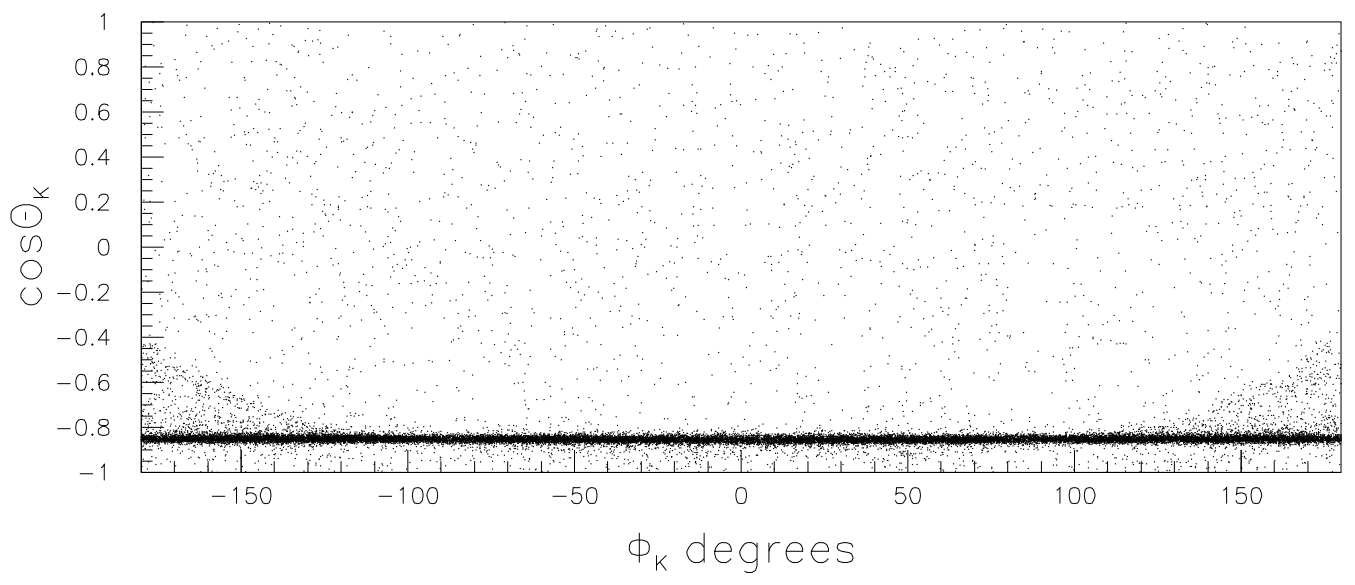

Figure 3.13: The $\cos \theta_{K}$ vs $\phi_{K}$ distribution for reconstructed data events.

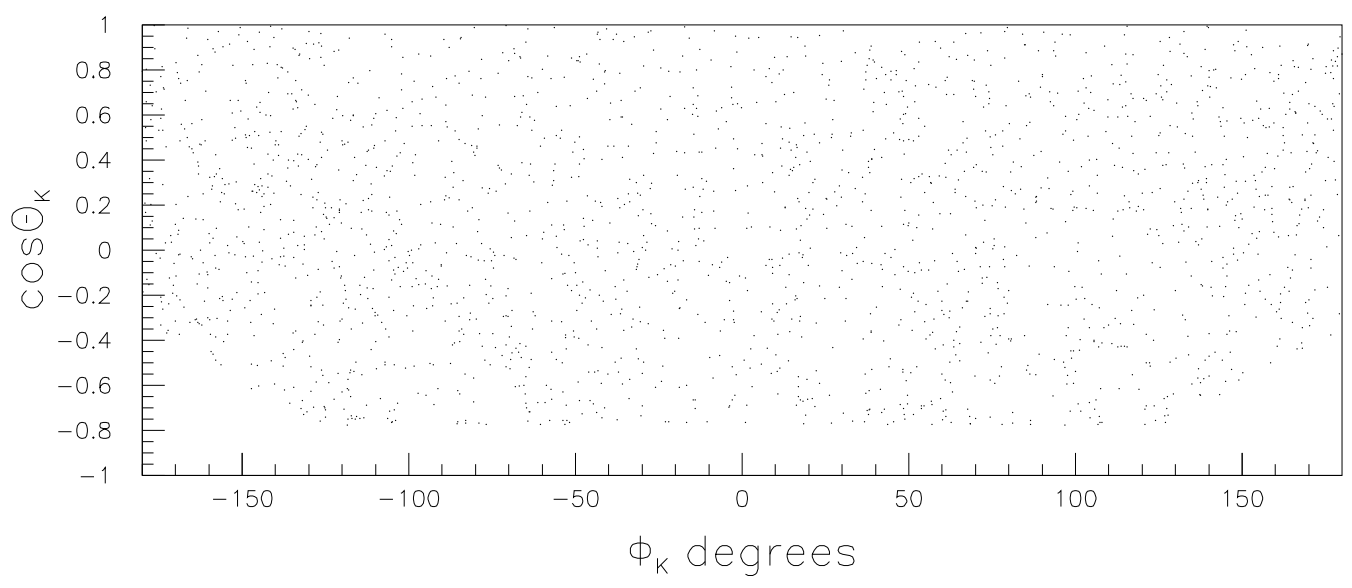

Figure 3.14: The $\cos \theta_{K}$ vs $\phi_{K}$ distribution for reconstructed data events after the kinematic angle cut. 

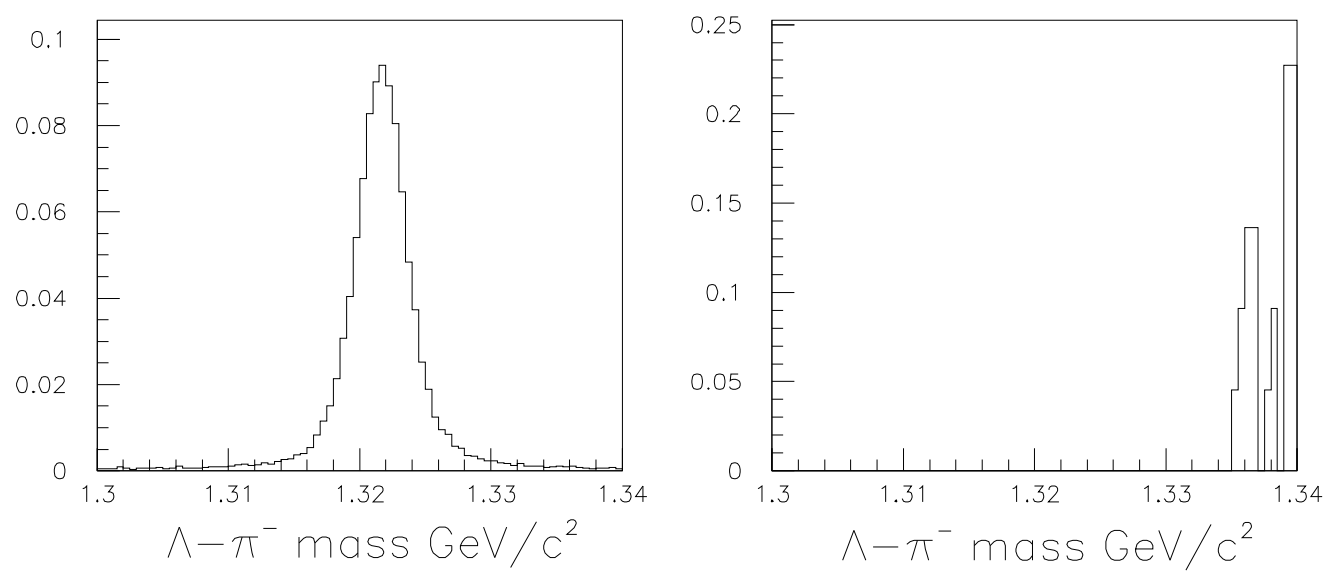

Figure 3.15: The $\Lambda^{-} \pi^{-}$invariant mass distribution for reconstructed data events before and after the kinematic angle cut.
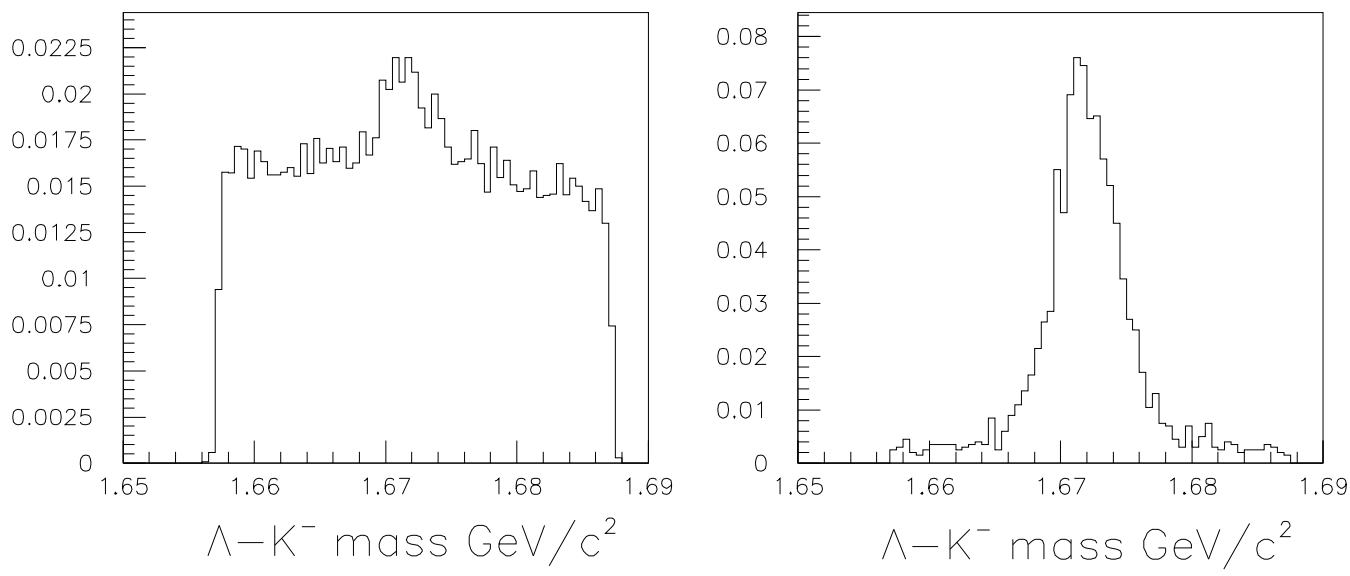

Figure 3.16: The $\Lambda$ - $\mathrm{K}^{-}$invariant mass distribution for reconstructed data events before and after the kinematic angle cut. 
mass distribution for $\Omega^{-}$events in the data sample and $\Omega^{-}$monte carlo events. However since the monte carlo generates events which reconstruct with a better resolution than the real data events we needed to model the resolution differences to separate this effect from the effects of background events. This was accomplished by normalizing the mass distributions of an essentially background free $\Xi^{-}$data sample and a sample of $\Xi^{-}$monte carlo events. The real $\Xi^{-}$distribution was divided bin by bin by the monte carlo $\Xi^{-}$distribution. This gave a multiplicative factor for each bin of the mass distribution. This factor is shown in Figure 3.17 the multiplier obtained from the $\Omega^{-}$ data and monte carlo distributions is also shown. By multiplying each bin of an $\Omega^{-}$ monte carlo mass distribution by the resolution correction factor and comparing the resulting mass distribution to the real $\Omega^{-}$mass distribution, as shown in Figure 3.18, the number of background events for each bin can be estimated. This method gives a total background estimate of approximately $3 \%$. This estimate agrees with the estimate from the monte carlo background studies from Table 3.4 and we will use the estimate from the mass distributions as the actual level of background in the $\Omega^{-}$sample.

The effect of the background and the background elimination cuts on the final polarization and magnetic moment results is discussed in chapter 5.

\subsection{Reconstructed Samples and Particle Yields}

The reconstructed sample $\Omega^{-}$and $\Xi^{-}$and the approximate yield per proton are in Table 3.5. The samples reflect the reconstruction selection criteria for both samples and the background reduction criteria for the $\Omega^{-}$sample. The yield estimates were made using rough estimates of average $\Omega^{-}$and $\Xi^{-}$per data run and approximate number of protons delivered to the proton center beamline during the run as measured by a secondary emission monitor (SEM). 

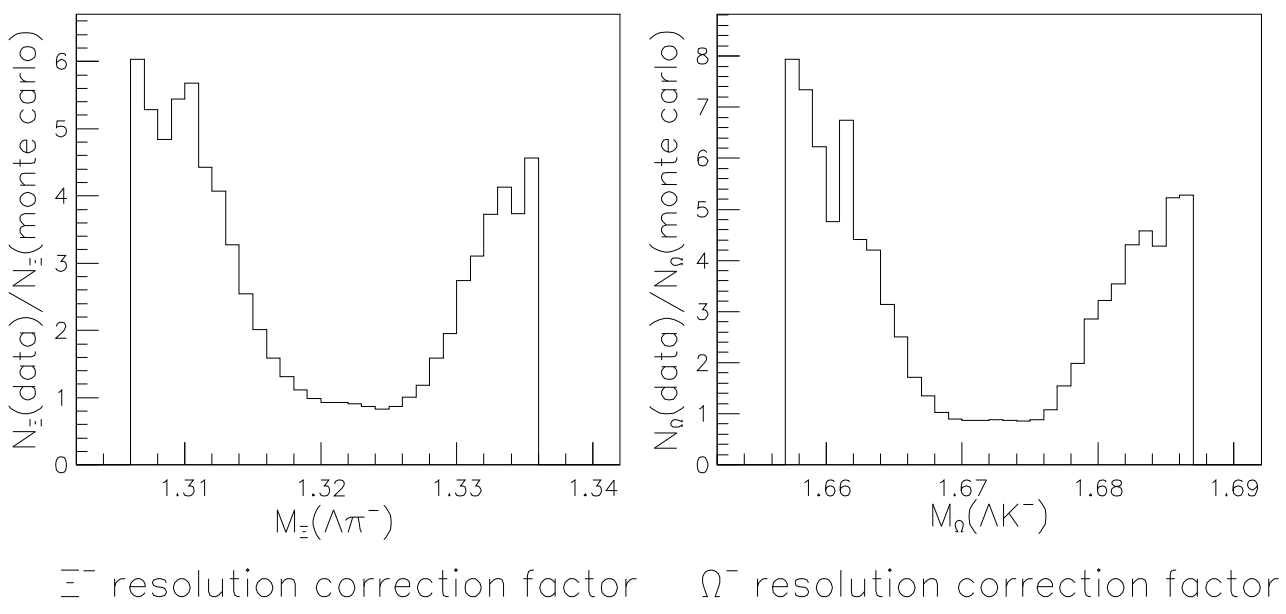

Figure 3.17: The multiplicative factor determined from the bin by bin division of the data and monte carlo $\Xi^{-}$and $\Omega^{-}$mass distributions. The similarity in the shapes of the two plots indicates that the shape is in fact due to resolution. The difference in the size of the multipliers shows that there is indeed background in the $\Omega^{-}$sample and none in the $\Xi^{-}$sample.

\begin{tabular}{l|c|c|c|c|c} 
Production & $\int \mathrm{Bdl}$ & \multicolumn{2}{|c|}{ Number of events } & \multicolumn{2}{|c}{ Yield per incident proton } \\
mode & $(\mathrm{T} \cdot \mathrm{m})$ & $\Omega^{-}$ & $\Xi^{-}$ & $\Omega^{-}$ & $\Xi^{-}$ \\
Neutral & -24.36 & 266700 & 29356174 & $\approx 10^{-11}$ & $\approx 10^{-9}$ \\
Neutral & -17.38 & 90277 & 10749346 & $\approx 8 \times 10^{-12}$ & $\approx 10^{-9}$ \\
Spin & -24.36 & 28994 & 2477187 & $\approx 3 \times 10^{-12}$ & $\approx 3 \times 10^{-10}$ \\
$0.0 \mathrm{mr}$ & -24.36 & 27837 & 3694258 & $\approx 2 \times 10^{-11}$ & $\approx 2 \times 10^{-9}$
\end{tabular}

Table 3.5: The total $\Omega^{-}$and $\Xi^{-}$samples and the approximate yields per incident proton. 


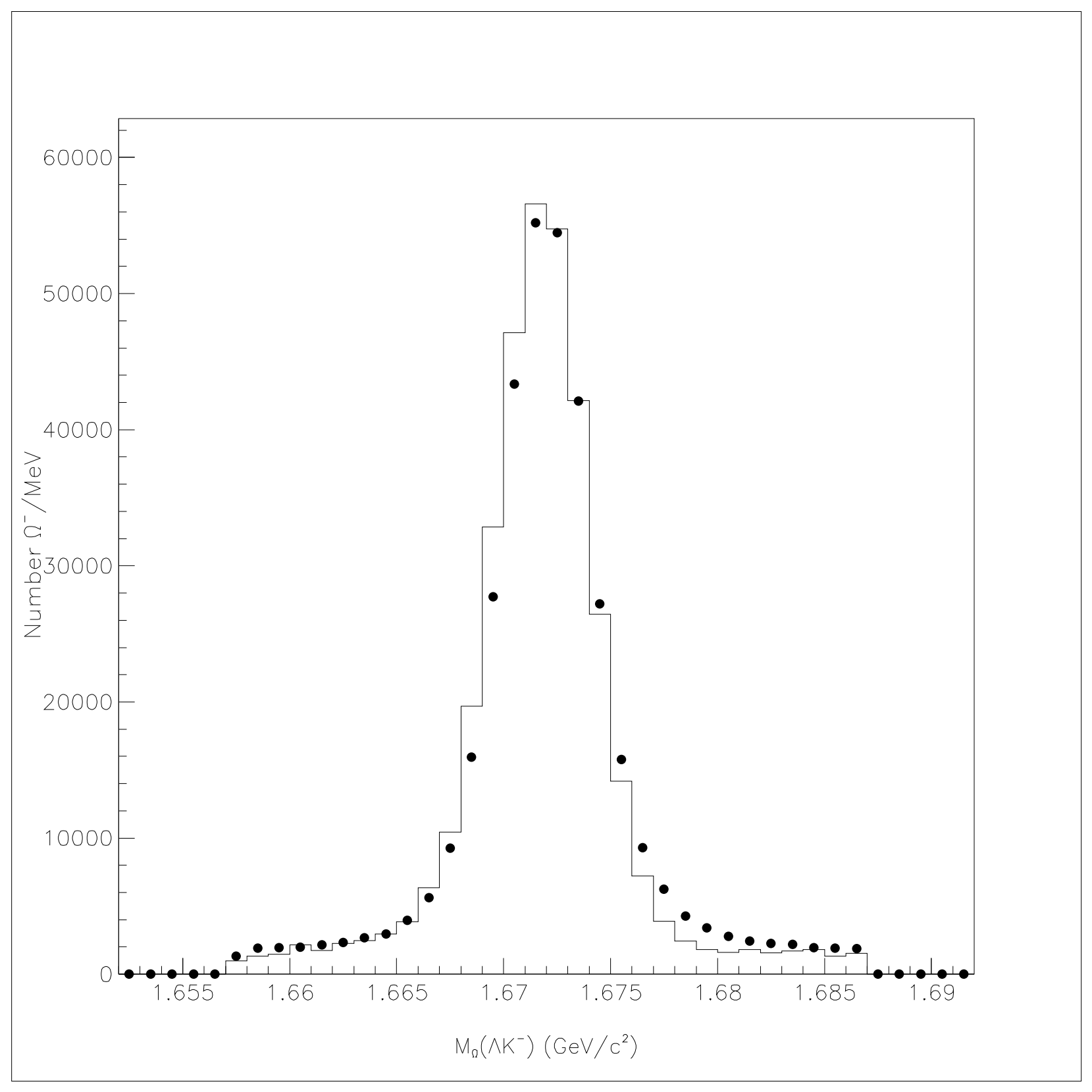

Figure 3.18: The $\Lambda$ - $\mathrm{K}^{-}$invariant mass distribution for reconstructed $\Omega^{-}$events. The histogram is a resolution corrected monte carlo mass distribution. The dots are the mass distribution for the entire $\Omega^{-}$data sample used for this analysis. 


\section{Chapter 4}

\section{Analysis}

\subsection{Magnetic Moment Analysis}

The magnetic moment in of an $\Omega^{-}$precessing in a magnetic field in nuclear magnetons is

$$
\mu_{\Omega^{-}}=\frac{m_{\text {proton }}}{\epsilon}\left(\frac{\Phi}{\int B d l}+\frac{-\epsilon}{m_{\Omega^{-}}}\right) S_{\Omega^{-}}
$$

where $\mathrm{m}_{\text {proton }}$ is the proton mass in $\mathrm{GeV} / \mathrm{c}^{2}$, e is the magnitude of the electron charge, $\Phi$ is the precession angle in radians, $\int \mathrm{Bdl}$ is the field integral of the precession magnet in Tesla-meters, $\mathrm{m}_{\Omega^{-}}$is the $\Omega^{-}$mass in $\mathrm{GeV}$ and $\mathrm{S}_{\Omega^{-}}$is the $\Omega^{-}$spin in units of $\hbar$. Using the appropriate values for the constants in the above equation we get,

$$
\mu_{\Omega^{-}}=-1.683\left(\frac{\Phi}{-.1790 \int B d l}+1\right)
$$

In chapter one it was shown that the magnetic moment can be obtained by measuring the final state polarization direction.

$$
\Phi=\tan ^{-1}\left(\frac{P_{z}}{P_{x}}\right)+n \pi
$$




\subsection{Omega Minus and Lambda Polarizations and Angu- lar Distributions}

Equations 4.1 and 1.19 show that the measurement of the magnetic moment is a measurement of the final state polarization components. It can be shown that the final state polarization can be measured by measuring the distribution of the daughter particles $[45,46,47]$. If we consider the distribution of the $\Lambda$ in the $\Omega^{-}$rest frame from the decay $\Omega^{-} \rightarrow \Lambda+\mathrm{K}^{-}$we find

$$
I(\theta, \phi)=A(\theta, \phi)+\alpha_{\Omega^{-}} B(\theta, \phi)
$$

where $\theta$ and $\phi$ are the polar angles of the $\Lambda$ direction in the lab coordinate system, $\alpha_{\Omega^{-}}$is the decay asymmetry parameter of the $\Omega^{-}$,

and

$$
A(\theta, \phi)=\sum_{L=0, \text { Leven }}^{2 J-1} \sum_{M=-L}^{L} n_{L 0}^{J} t_{L M} Y_{L M}^{*}(\theta, \phi)
$$

is the even parity part of the decay distribution and

$$
B(\theta, \phi)=\sum_{L=1, L o d d}^{2 J-1} \sum_{M=-L}^{L} n_{L 0}^{J} t_{L M} Y_{L M}^{*}(\theta, \phi)
$$

is the parity non-conserving part of the decay distribution. Where $\mathrm{J}$ is the $\Omega^{-}$spin and $\mathrm{L}$ is the relative angular momentum of the decay products. The $\mathrm{Y}_{L M}$ are the spherical harmonics. The $\mathrm{n}_{L 0}^{J}$ are normalization constants proportional to the ClebshGordan coefficients and are given by

$$
n_{L 0}^{J}=(-1)^{J-1 / 2} \sqrt{\frac{2 J+1}{4 \pi}}\left\langle J J \frac{1}{2} \frac{1}{2} \mid L 0\right\rangle
$$

The $\mathrm{t}_{L M}$ are the elements of the spin density matrix related to the expectation values 
of the spin components of the $\Omega^{-}$

$$
t_{L M}=\sum_{m m^{\prime}}\left\langle J m \mid J L m^{\prime} M\right\rangle \rho_{m m^{\prime}}
$$

Where $\mathrm{J}$ is the total angular momentum of the $\Omega^{-}$in units of $\hbar(3 / 2)$, and $\rho_{m m^{\prime}}$ is the $\mathrm{mm}$ ' element of the spin density matrix for the $\Omega^{-}$.

Since the $\Lambda$ polarization, $\vec{P}_{\Lambda}$, is of odd parity the integral

$$
\int I(\theta, \phi) \vec{P}_{\Lambda} \cdot \hat{\Lambda} \sum_{L=0}^{\infty} \sum_{M=-L}^{L} Y_{L M} d \Omega
$$

gives the coefficients of the multipole expansion for the longitudinal $\Lambda$ polarization and we find

$$
I(\theta, \phi) \vec{P}_{\Lambda} \cdot \hat{\Lambda}=\alpha_{\Omega^{-}} A(\theta, \phi)+B(\theta, \phi)
$$

where, $\hat{\Lambda}$ is a unit vector in the direction of the $\Lambda$ momentum and the definitions of I, A, and $\mathrm{B}$ are given above.

Similarly the orthogonal components of the polarization are given by

$$
\begin{aligned}
& I(\theta, \phi) \vec{P}_{\Lambda} \cdot(\hat{X}+i \hat{Y})= \\
& \left(i \beta_{\Omega^{-}}-\gamma_{\Omega^{-}}\right)(2 J+1) \sum_{L=1, \text { Lodd }}^{2 J} \sum_{m=-L}^{L} \sqrt{\frac{2 L+1}{4 \pi(L(L+1))}} n_{L 0}^{J} t_{L M} D_{M 1}^{L}(\phi, \theta, 0)(
\end{aligned}
$$

where $\gamma_{\Omega^{-}}$and $\beta_{\Omega^{-}}$are decay parameters of the $\Omega^{-}$and the $\mathrm{D}_{M 1}^{L}$ are the Wigner rotation matrices. $\hat{X}$ is defined with respect to the laboratory quantization direction, $\hat{z}$, as

$$
\hat{X}=\frac{\hat{\Lambda} \times(\hat{\Lambda} \times \hat{z})}{|\hat{\Lambda} \times(\hat{\Lambda} \times \hat{z})|}
$$

similarly $\hat{Y}$ is

$$
\hat{Y}=\frac{\hat{z} \times \hat{\Lambda}}{|\hat{z} \times \hat{\Lambda}|}
$$


the relationship between the lab axes $\hat{x}, \hat{y}$, and $\hat{z}$ to $\hat{X}, \hat{Y}$, and $\hat{\Lambda}$ is shown in Figure 4.1.

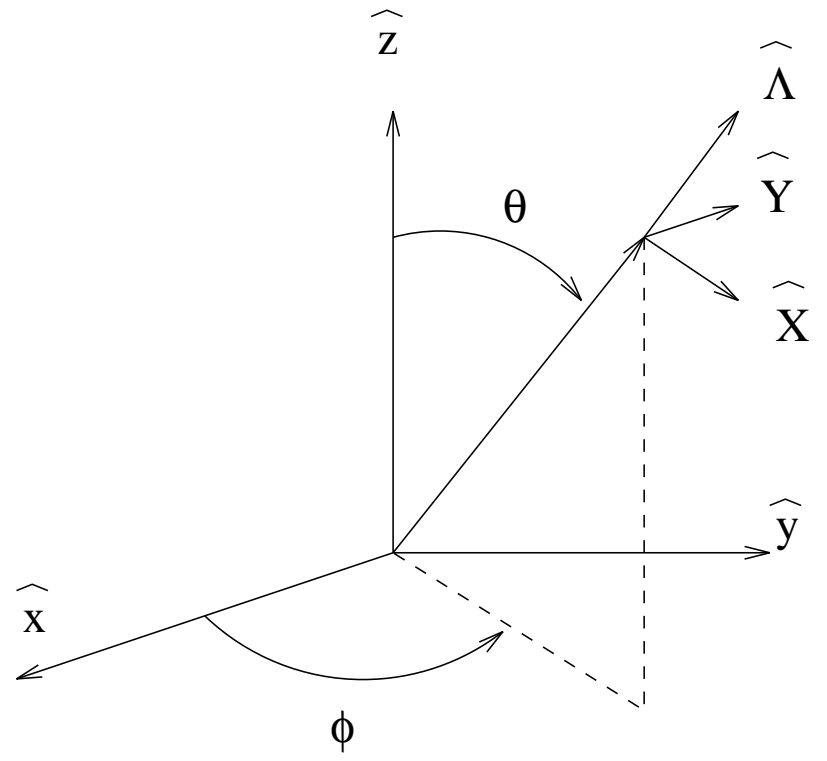

Figure 4.1: The relationship of the lab frame $(\hat{x}, \hat{y}, \hat{z})$ to the $\Lambda$ frame $(\hat{X}, \hat{Y}, \hat{\Lambda})$ showing the polar angles $\theta$ and $\phi$

The vector (rank 1) polarization components can be expressed in terms of the $t_{1 M}$ with $\mathrm{M}=-1,0$, or 1 by

$$
\begin{gathered}
P_{x}=\sqrt{\frac{J+1}{J}}\left(\frac{t_{1-1}-t_{11}}{\sqrt{2}}\right) \\
P_{y}=i \sqrt{\frac{J+1}{J}}\left(\frac{t_{1-1}+t_{11}}{\sqrt{2}}\right) \\
P_{z}=\sqrt{\frac{J+1}{J}} t_{10}
\end{gathered}
$$

The decay $\Lambda \rightarrow \pi^{-}+\mathrm{p}$ proceeds via the weak interaction with a branching ratio of $64 \%$. The spacial distribution of the protons in the $\Lambda$ frame is 


$$
\frac{1}{N_{p}} \frac{d N_{p}}{d \Omega_{p}}=\frac{1}{4 \pi}\left(1+\alpha_{\Lambda} \vec{P}_{\Lambda} \cdot \hat{p}\right)
$$

where $\Omega_{p}$ is the solid angle, $\vec{P}_{\Lambda}$ is the direction of the $\Lambda$ polarization, and $\hat{p}$ is the unit vector in the direction of the proton momentum. $\alpha_{\Lambda}$ has been measured as +0.642 \pm 0.013 [36]. Since Equation 4.16 is independent of azimuthal angle the distribution can be expressed in terms of an arbitrary unit vector, $\hat{n}$, as

$$
\frac{1}{N_{p}} \frac{d N_{p}}{d \cos \theta_{p}}=\frac{1}{4 \pi}\left(1+\alpha_{\Lambda} \vec{P}_{\Lambda} \cdot \hat{n} \cos \theta_{p}\right)
$$

where $\cos \theta_{p}=\hat{n} \cdot \hat{p}$. By choosing $\hat{n}$ parallel to the laboratory axes the $\Lambda$ polarization can be determined. The angular distribution of the proton in the $\Omega^{-}$frame is the product of the distribution of the $\Lambda$ in the $\Omega$ rest frame and the proton distribution in the $\Lambda$ frame

$$
I_{\Lambda}\left(\theta_{\Lambda}, \phi_{\Lambda}\right) I_{p}\left(\theta_{p}, \phi_{p}\right)=\frac{1}{4 \pi}\left(I_{\Lambda}+\alpha_{\Lambda} I_{\Lambda} \vec{P}_{\Lambda} \cdot \hat{X} \hat{X} \cdot \hat{p}+\alpha_{\Lambda} I_{\Lambda} \vec{P}_{\Lambda} \cdot \hat{Y} \hat{Y} \cdot \hat{p}\right)
$$

If we express the proton distribution in terms of the angles in the $\Lambda$ coordinate system as shown in Figure 4.2 we can integrate the above distribution over the azimuthal angle of the proton, $\phi_{p}$, and the solid angle of the $\Lambda$ decay in the $\Omega^{-}$frame, $\Omega_{\Lambda}$, which yields the proton distribution.

$$
\frac{1}{N_{p}} \frac{d N_{p}}{d \cos \theta_{p}}=\frac{1}{2}\left(1+\alpha_{\Omega^{-}} \alpha_{\Lambda} \cos \theta_{p}\right)
$$

Projecting the $\Lambda$ coordinate system onto the lab system and integrating over $\phi_{p}$ and $\Omega_{\Lambda}$ gives another expression for the proton distribution,

$$
\frac{1}{N_{p}} \frac{d N_{p}}{d \cos \theta_{p}}=\frac{1}{2}\left(1+\frac{\alpha_{\Lambda}}{(2 J+1)}\left[1+2 \gamma_{\Omega^{-}}(2 J+1)\right] \vec{P}_{\Omega^{-}} \cdot \hat{p}\right)
$$




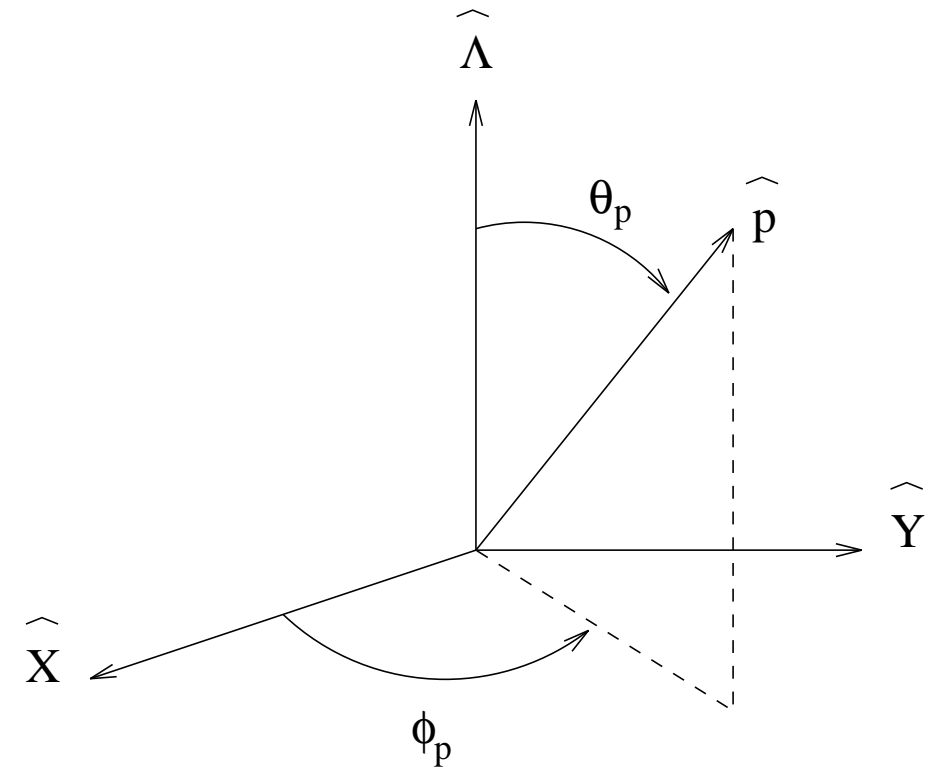

Figure 4.2: The proton direction in the $\Lambda$ coordinate system showing the polar angles $\theta_{p}$ and $\phi_{p}$

By comparing the equation above to Equation 4.14 we can relate the $\Lambda$ polarization to the $\Omega^{-}$polarization. The relation is

$$
\overrightarrow{P_{\Lambda}}=\frac{1}{2(J+1)}\left[1+(2 J+1) \gamma_{\Omega^{-}}\right] \vec{P}_{\Omega}
$$

Thus component by component the $\Omega^{-}$polarization is proportional to the $\Lambda$ polarization. The value of $\gamma_{\Omega^{-}}$has not been measured, however time reversal symmetry requires $\beta_{\Omega^{-}}=0$ and $\alpha_{\Omega^{-}}$has been measured to be close to zero $\left(\alpha_{\Omega^{-}}=0.026 \pm\right.$ 0.026) [36]. Applying the normalization condition,

$$
\alpha_{\Omega^{-}}^{2}+\beta_{\Omega^{-}}^{2}+\gamma_{\Omega^{-}}^{2}=1
$$

we can surmise that $\left|\gamma_{\Omega^{-}}\right|=1$. Using this information and setting $\mathrm{J}=3 / 2$ for the $\Omega^{-}$we find the following

$$
\overrightarrow{P_{\Lambda}}=\vec{P}_{\Omega}
$$


for $\gamma_{\Omega^{-}}=1$, or

$$
\overrightarrow{P_{\Lambda}}=-0.6 \vec{P}_{\Omega}
$$

for $\gamma_{\Omega^{-}}=-1$. Since the magnetic moment depends only on the ratio of two polarization components the measurement is not effected by the value of $\gamma_{\Omega^{-}}$.

The proportionality of the $\Omega^{-}$and $\Lambda$ polarizations, reduces the measurement of $\mu_{\Omega}$ to the measurement of the polarization components of the daughter $\Lambda$.

\subsubsection{Lambda Polarization}

The decay distribution for the proton from the decay $\Lambda \rightarrow \mathrm{p}+\pi^{-}$with respect to the laboratory axis $\mathrm{i}, \theta_{i}$ is given by

$$
\frac{d N_{p}}{d\left(\cos \theta_{i}\right)}=\frac{1}{2}\left(1+\alpha_{\Lambda} P_{\Lambda_{i}} \cos \theta_{i}\right)
$$

Where $\mathrm{P}_{\Lambda_{i}}$ is the ith component of the $\Lambda$ polarization. If the spatial acceptance of the experiment was perfect then the components of the $\Lambda$ polarization could be determined by fitting the distribution shown in Equation 4.25 and finding the best value of $\mathrm{P}_{\Lambda_{i}}$. Figure 4.3 shows the $\cos \theta_{p}$ distributions for a sample of $\Omega^{-}$events. The distributions are not linear in all regions of the $\cos \theta_{p}$ distributions. The depleted regions at small angles in $\mathrm{z}$, which also correspond to the depleted regions in $\mathrm{x}$ and $\mathrm{y}$ demonstrate the less than perfect acceptance of the E 800 spectrometer and reconstruction program. More sophisticated methods of determining the $\Lambda$ polarization compensate for the imperfect acceptance of the spectrometer and reconstruction program.

\subsection{The Acceptance Cancellation Method}

The acceptance cancellation method is motivated by observing that the direction of the polarization should change with the sign of the targeting angle, while the acceptance 

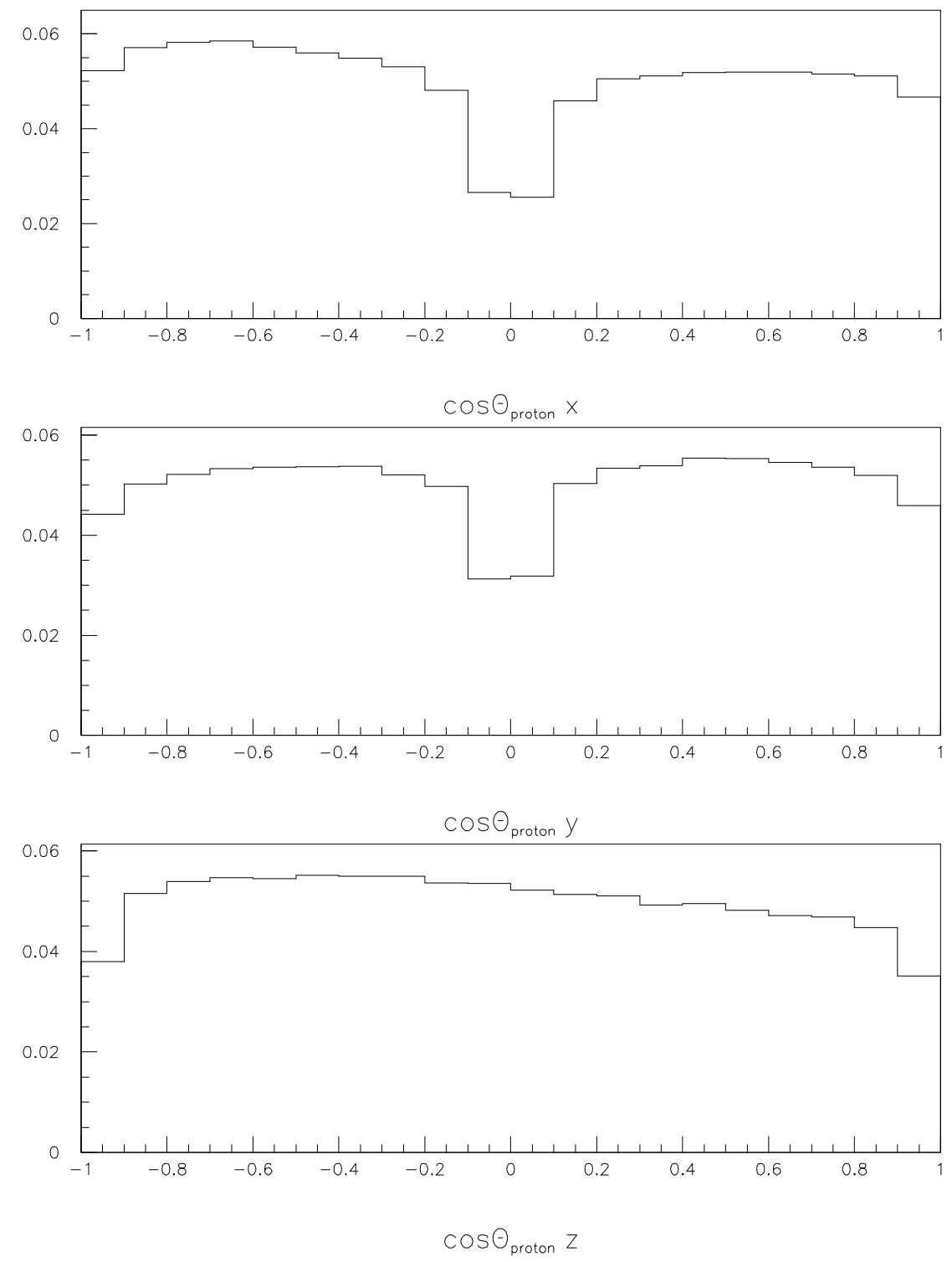

Figure 4.3: The proton distributions for $\mathrm{x}, \mathrm{y}$ and $\mathrm{z}$ for a sample of $\Omega^{-}$events. 
of the spectrometer should, for the most part, remain constant. The distributions for positive and negative angles $\mathrm{N}_{p}^{+}$and $\mathrm{N}_{p}^{-}$can be expressed as

$$
\frac{d N_{p}^{+}}{d\left(\cos \theta_{i}\right)}=\frac{1}{2} ?\left(\cos \theta_{i}, \phi\right)\left(1+\alpha_{\Lambda} P_{\Lambda_{i}} \cos \theta_{i}\right)
$$

and

$$
\frac{d N_{p}^{-}}{d\left(\cos \theta_{i}\right)}=\frac{1}{2} ?\left(\cos \theta_{i}, \phi\right)\left(1-\alpha_{\Lambda} P_{\Lambda_{i}} \cos \theta_{i}\right)
$$

where $?\left(\cos \theta_{i}, \phi\right)$ is the acceptance factor. If $?\left(\cos \theta_{i}, \phi\right)$ remains the same for both targeting angles then the $\Lambda$ polarization can be computed from

$$
\left(\frac{N^{+}-N^{-}}{N^{+}+N^{-}}\right)_{i}=\alpha_{\Lambda} P_{\Lambda_{i}} \cos \theta_{i}
$$

using a bin by bin subtraction of the distributions.

However, since the beam distributions are no the same for both targeting angles the acceptance of the spectrometer may not be equal for both positive and negative targeting angles another method which models the acceptance of the apparatus more accurately has been developed.

\subsection{The Hybrid Monte Carlo Technique}

The hybrid monte carlo technique generates monte simulated events based on real events to map the acceptance in $\cos \theta_{i}$. The monte carlo events are generated using the same total momenta and vertices as the real event and generating a random $\cos \theta_{i}$ between -1 and +1 . The monte carlo events are subject to same selection criteria and geometric acceptance as the real events. Monte carlo events are generated such that thirty fake events are accepted for each real event. This number of monte carlo events gives a stable solution and gives a statistical uncertainty which can be neglected compared to the statistical error in the data sample. 
For a data sample with zero polarization, $\vec{P}_{\Lambda}=0$, the monte carlo $\cos \theta_{i}$ distributions should match those of the data since they are subject to the same acceptance criteria. If there is a polarization in the data sample the distributions will not match due to the asymmetry in the data distribution from the polarization signal. To correct for this mismatch each fake event is weighted by a factor

$$
W_{i j k}=\frac{1+\alpha_{\Lambda} P_{\Lambda_{i}} \cos \theta_{i j k}}{1+\alpha_{\Lambda} P_{\Lambda_{i}} \cos \theta_{i j}}
$$

where i refers to the component of the polarization, the subscript $\mathrm{j}$ refers to the real event, and $\mathrm{jk}$ refers to the kth monte-carlo event generated from real event $\mathrm{j}$. For each bin the real and monte-carlo distributions are compared and a $\chi^{2}$ is generated for each bin

$$
\chi^{2}=\frac{\left(n_{r}-n_{f} N_{R} / N_{f}\right)^{2}}{n_{r}}
$$

where $\mathrm{n}_{r}$ and $\mathrm{n}_{f}$ are the contents of the bins of the real and fake distributions, and $\mathrm{N}_{r}$ and $\mathrm{N}_{f}$ are the total number of real and monte-carlo events. For a given bin $\mathrm{n}_{f}$ is:

$$
n_{f}=\sum_{j k} W_{j k}
$$

The total $\chi^{2}$ is calculated by summing the $\chi^{2}$ for each bin. $\alpha_{\Lambda} \mathrm{P}_{\Lambda_{i}}$ for a given targeting angle is calculated by minimizing the total $\chi^{2}$ with respect to $\alpha_{\Lambda} \mathrm{P}_{\Lambda_{i}}$. The error in the polarization is calculated by finding the values of $\mathrm{P}_{\Lambda_{i}}$ on either side of the minimum $\chi^{2}$ which increase the $\chi^{2}$ by 1 .

\subsubsection{Analysis Selection Criteria}

In using the hybrid monte carlo technique there is a possibility that the fake events generated by the program may not be completely representative of the data events. There are two possible sources of this problem. First the reconstruction could reconstruct 
some data events into the wrong $\cos \theta$ bin. Figure 4.4 shows the difference between the generated and reconstructed $\cos \theta_{p}$ for all axes. The plots have a logarithmic scale and indicate that $99.5 \%$ of the events are reconstructed into the correct $\cos \theta$ bin.

The second possibility is that some classes of data events are not accepted and reconstructed with $100 \%$ efficiency. In most cases these are events which have small spatial separation in the chambers and thus are close to the small angle resolution of the apparatus and reconstruction program. This creates a situation where there is an excess of fake events in some $\cos \theta$ bins and results in very high $\chi^{2}$ for the asymmetry fits. By digitizing the tracks for the fake events we can select events where the tracks are well separated in the chambers for both the data and hybrid monte-carlo fake events. These selection criteria are chosen by studying the $\cos \theta_{p}$ distributions for the fake and real events in the small angle bins. The effects of these selection criteria on the polarization and magnetic moment results will be discussed in the systematic studies section of Chapter 5 .

\subsubsection{Biases and Polarization}

In general, the asymmetries computed using the hybrid monte carlo technique may be composed of two terms, the polarization signal, which changes sign with targeting angle, and a "bias" term, which does not change sign. The bias term is due to unknown imperfections in our knowledge of the acceptance of the apparatus and reconstruction program. The measured asymmetry, $\mathrm{A}_{i}^{ \pm}$, can be expressed as

$$
A_{i}^{ \pm}=B_{i} \pm \alpha_{\Lambda} P_{\Lambda_{i}}
$$

where the $B_{i}$ are the bias components and the \pm indicates the positive or negative targeting angle. The polarization and bias components can be computed by finding the difference and sum of the signals from the positive and negative targeting angles in the 

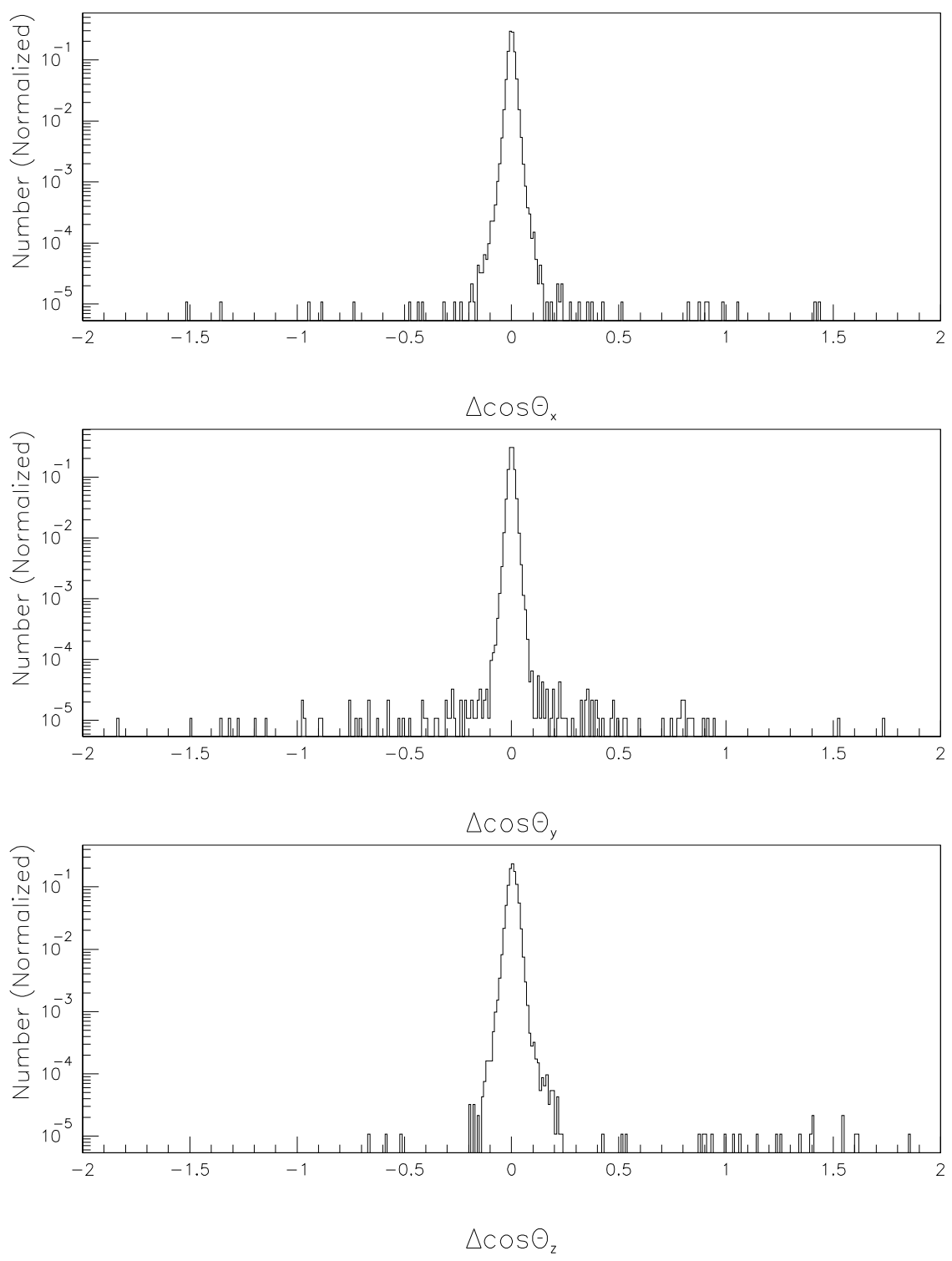

Figure 4.4: The difference between the generated and reconstructed $\cos \theta_{p}$ for $\mathrm{x}, \mathrm{y}, \mathrm{z}$. 
following manner:

$$
\alpha_{\Lambda} P_{\Lambda_{i}}=\frac{A_{i}^{+}-A_{i}^{-}}{2}
$$

and

$$
B_{i}=\frac{A_{i}^{+}+A_{i}^{-}}{2}
$$

Using Equation 4.31 to compute the $\Lambda$ polarization cancels the bias terms. Equation 4.32 gives the bias terms themselves, which indicate how well the acceptance of the apparatus, trigger, and reconstruction have been reproduced in the hybrid monte carlo. The biases tend to depend on the $\Omega^{-}$momentum as shown in Figure 5.2. This dependence is the result of the relationship between the parent momentum and the opening angle of the $\Lambda$ decay.

\subsection{Performance of the Polarization Analysis}

The performance of the polarization analysis can be judged by using unpolarized and polarized monte carlo samples and comparing the input and output polarizations. Table 4.1 shows the input and output polarizations for two samples of monte carlo $\Omega^{-}$. These samples were analyzed directly without invoking the reconstruction program. This was accomplished by tapping out the generated momentum vectors and decay vertices for each event and reading these quantities directly into the polarization analysis. Table 4.2 contains the same data but the analysis includes output resulting from reconstructing the events and inputting the resulting momentum vectors and vertices into the analysis program. Both of the results agree with the input polarizations to within statistical uncertainties.

\subsection{The Master Fit}

It is possible to combine several samples of data taken in different targeting modes or with different precession fields by minimizing the $\chi^{2}$ given by 


\begin{tabular}{c|c|c|c|c|c}
$\begin{array}{c}\text { Input } \\
\text { Polarization }\end{array}$ & Component & $\begin{array}{c}\text { Output } \\
\text { Polarization }\end{array}$ & Bias & \multicolumn{2}{|c}{$\chi^{2}$} \\
\hline 0.000 & $\mathrm{x}$ & $0.0050 \pm 0.0045$ & $0.0037 \pm 0.0045$ & 28.0 & 12.7 \\
0.000 & $\mathrm{y}$ & $-0.0034 \pm 0.0049$ & $-0.0043 \pm 0.0049$ & 21.5 & 33.4 \\
0.000 & $\mathrm{z}$ & $-0.0014 \pm 0.0053$ & $0.0029 \pm 0.0053$ & 15.2 & 9.4 \\
\hline 0.016 & $\mathrm{x}$ & $0.0177 \pm 0.0037$ & $-0.0032 \pm 0.0042$ & 17.5 & 9.2 \\
0.000 & $\mathrm{y}$ & $0.0051 \pm 0.0040$ & $0.0007 \pm 0.0040$ & 23.6 & 19.7 \\
0.022 & $\mathrm{z}$ & $0.0263 \pm 0.0043$ & $0.0042 \pm 0.0043$ & 25.1 & 16.0 \\
\hline
\end{tabular}

Table 4.1: The input and output polarizations, biases, and $\chi^{2}$ 's for unreconstructed $\Omega^{-}$ monte-carlo samples. The $\chi^{2}$ 's are based on 19 degrees of freedom for the $20 \cos \theta_{p}$ bins in each view.

\begin{tabular}{c|c|c|c|c|c}
$\begin{array}{c}\text { Input } \\
\text { Polarization }\end{array}$ & Component & $\begin{array}{c}\text { Output } \\
\text { Polarization }\end{array}$ & Bias & \multicolumn{2}{|c}{$\chi^{2}$} \\
\hline 0.000 & $\mathrm{x}$ & $-0.0027 \pm 0.0061$ & $0.0218 \pm 0.0061$ & 41.6 & 18.4 \\
0.000 & $\mathrm{y}$ & $-0.0026 \pm 0.0067$ & $-0.0004 \pm 0.0067$ & 40.1 & 34.9 \\
0.000 & $\mathrm{z}$ & $0.0018 \pm 0.0073$ & $0.0269 \pm 0.0018$ & 20.1 & 15.1 \\
\hline 0.016 & $\mathrm{x}$ & $0.0206 \pm 0.0050$ & $0.0043 \pm 0.0050$ & 24.0 & 34.5 \\
0.000 & $\mathrm{y}$ & $0.0075 \pm 0.0055$ & $0.0044 \pm 0.0055$ & 35.7 & 30.2 \\
0.022 & $\mathrm{z}$ & $0.0270 \pm 0.0059$ & $0.0107 \pm 0.0059$ & 19.7 & 21.9 \\
\hline
\end{tabular}

Table 4.2: The input and output polarizations, biases, and $\chi^{2}$ 's for reconstructed $\Omega^{-}$ monte-carlo samples. The $\chi^{2}$ 's are based on 19 degrees of freedom for the $20 \cos \theta_{p}$ bins in each view. 


$$
\chi^{2}=\sum_{i j}\left(\frac{P_{x_{i j}}-P_{t g t_{i j}} \cos \Phi_{j}}{\sigma_{x_{i j}}^{2}}\right)^{2}+\left(\frac{P_{z_{i j}}-P_{t g t_{i j}} \sin \Phi_{j}}{\sigma_{z_{i j}}^{2}}\right)^{2},
$$

with $\Phi_{j}$ related to the precession angle by Eq. $1.26 . \mathrm{P}_{t g t_{i j}}$ is the initial polarization at the target, which does not depend on the precession field but does change with production method. $\mathrm{P}_{x_{i j}}$ and $\mathrm{P}_{z_{i j}}$ are the measured $\mathrm{x}$ and $\mathrm{z}$ polarization components and $\sigma_{x_{i j}}^{2}$ and $\sigma_{z_{i j}}^{2}$ include uncertainties from $\mathrm{P}_{x_{i j}}$ and $\mathrm{P}_{z_{i j}}$. The sum is over $\mathrm{i}$ production methods, $\mathrm{j}$ field integrals. By constraining the magnetic moment to be the same for all samples, the data from various samples was combined to generate a single magnetic moment result. Errors were found by varying the magnetic moment until the $\chi^{2}$ increases by one from the minimum value. 


\section{Chapter 5}

\section{Results and Systematic Uncertainty Studies}

\subsection{Omega Minus Polarization Results}

All the data for this dissertation were reconstructed and analyzed for polarization using the analysis programs described in the previous chapters. The data samples which comprise the final $\Omega^{-}$data set are listed in Table 5.1.

\begin{tabular}{l|c|c|c} 
Production mode & $\int \mathrm{Bd} 1(\mathrm{~T} \cdot \mathrm{m})$ & Targeting angles $(\mathrm{mr})$ & $10^{3}$ events \\
\hline Neutral & -24.36 & \pm 1.8 & 166.5 \\
Neutral & -17.38 & \pm 1.8 & 50.1 \\
Spin Transfer & -24.36 & \pm 1.8 & 18.3 \\
$0.0 \mathrm{mr}$ & -24.36 & 0.0 & 17.5
\end{tabular}

Table 5.1: The data sets making up the final $\Omega^{-}$data sample.

Table 5.2 gives the measured polarization, biases, and $\chi^{2}$ for four momentum bins, for the neutral production sample $\left(\int \mathrm{Bdl}=-24.36 \mathrm{~T} \cdot \mathrm{m}\right)$ using the hybrid monte carlo technique as a function of momentum. Table 5.3 gives the momentum averaged values for each of the data samples.

The polarization results for the momentum bins for the neutral production sample 


\begin{tabular}{c|c|c|c|c|c}
$\begin{array}{c}\text { Mom. }(\mathrm{GeV} / \mathrm{c}) \\
10^{3} \text { Events }\end{array}$ & View & Polarization & Bias & \multicolumn{2}{|c}{$\chi^{2}$} \\
\hline 348 & & $\alpha_{\Lambda} \mathrm{P}_{\Lambda}$ & & + & - \\
31.3 & $\mathrm{x}$ & $0.0215 \pm 0.0092$ & $-0.0092 \pm 0.0092$ & 17.8 & 23.1 \\
& $\mathrm{y}$ & $-0.0005 \pm 0.0097$ & $0.0151 \pm 0.0097$ & 38.5 & 32.3 \\
& $\mathrm{z}$ & $0.0255 \pm 0.0115$ & $0.0208 \pm 0.0115$ & 19.4 & 11.0 \\
\hline 380 & $\mathrm{x}$ & $0.0048 \pm 0.0084$ & $0.0086 \pm 0.0084$ & 28.8 & 19.3 \\
35.6 & $\mathrm{y}$ & $0.0125 \pm 0.0094$ & $0.0363 \pm 0.0094$ & 18.3 & 26.2 \\
& $\mathrm{z}$ & $0.0204 \pm 0.0101$ & $0.0394 \pm 0.0101$ & 15.1 & 37.9 \\
\hline 403 & $\mathrm{x}$ & $0.0207 \pm 0.0090$ & $-0.0006 \pm 0.0090$ & 16.6 & 21.2 \\
31.2 & $\mathrm{y}$ & $0.0117 \pm 0.0099$ & $-0.0042 \pm 0.0099$ & 12.0 & 17.7 \\
& $\mathrm{z}$ & $0.0311 \pm 0.0103$ & $0.0524 \pm 0.0103$ & 8.8 & 19.4 \\
\hline 443 & $\mathrm{x}$ & $0.0272 \pm 0.0086$ & $0.0150 \pm 0.0086$ & 28.4 & 36.3 \\
33.2 & $\mathrm{y}$ & $0.0065 \pm 0.0090$ & $0.0088 \pm 0.0090$ & 34.4 & 18.3 \\
& $\mathrm{z}$ & $0.0149 \pm 0.0100$ & $0.0766 \pm .0100$ & 46.7 & 42.7 \\
\hline
\end{tabular}

Table 5.2: The measured $\Omega^{-}$polarizations, biases, and $\chi^{2}$ 's for the neutral production sample with $\int \mathrm{Bdl}=24.36 \pm 0.24 \mathrm{~T} \mathrm{~m}$. The $\chi^{2}$ 's are based on 20 degrees of freedom for the $20 \cos \theta_{p}$ bins in each view.

\begin{tabular}{c|c|c|c|c|c|c} 
Sample type & $\begin{array}{c}\text { Mom. (GeV/c) } \\
10^{3} \text { Events }\end{array}$ & View & $\begin{array}{c}\text { Polarization } \\
\alpha_{\Lambda} \mathrm{P}_{\Lambda}\end{array}$ & \multicolumn{2}{|c}{ Bias } & \multicolumn{2}{|c}{$\chi^{2}$} \\
\hline Neutral & 393 & $\mathrm{x}$ & $0.0179 \pm 0.0044$ & $0.0044 \pm 0.0044$ & 22.3 & 42.5 \\
Prod. & 166.5 & $\mathrm{y}$ & $0.0073 \pm 0.0048$ & $0.0141 \pm 0.0048$ & 15.3 & 32.8 \\
$(-24.36)$ & & $\mathrm{z}$ & $0.0218 \pm 0.0053$ & $0.0496 \pm 0.0053$ & 27.2 & 36.2 \\
\hline Neutral & 282 & $\mathrm{x}$ & $0.0186 \pm 0.0083$ & $-0.0032 \pm 0.0083$ & 6.0 & 15.1 \\
Prod. & 50.2 & $\mathrm{y}$ & $0.0072 \pm 0.0093$ & $0.0263 \pm 0.0093$ & 13.4 & 30.8 \\
$(-17.48)$ & & $\mathrm{z}$ & $0.0140 \pm 0.0109$ & $-0.0182 \pm 0.0114$ & 33.0 & 30.0 \\
\hline Spin- & 393 & $\mathrm{x}$ & $-0.028 \pm 0.013$ & $0.010 \pm 0.013$ & 15.2 & 22.9 \\
transfer & 18.3 & $\mathrm{y}$ & $0.007 \pm 0.014$ & $-0.008 \pm 0.014$ & 25.7 & 36.5 \\
Prod & & $\mathrm{z}$ & $-0.034 \pm 0.016$ & $0.043 \pm 0.016$ & 12.2 & 23.5 \\
\hline & 393 & $\mathrm{x}$ & $-0.006 \pm 0.014$ & $-0.008 \pm 0.014$ & 29.7 & 21.2 \\
$0.0 \mathrm{mr}$ & 19.6 & $\mathrm{y}$ & $-0.009 \pm 0.015$ & $-0.001 \pm 0.015$ & 4.6 & 14.6 \\
Prod & & $\mathrm{z}$ & $-0.017 \pm 0.016$ & $0.034 \pm 0.016$ & 24.1 & 19.6 \\
\hline
\end{tabular}

Table 5.3: The momentum averaged measured $\Omega^{-}$polarizations, biases, and $\chi^{2}$ 's for the three production methods. The $\chi^{2}$ 's are based on 20 degrees of freedom for the 20 $\cos \theta_{p}$ bins in each view. 
$\left(\int \mathrm{Bdl}=-24.36\right)$ and the momentum averaged values for all four configurations are shown in Figure 5.1. The biases are shown in Figure 5.2.

\subsection{Omega Minus Magnetic Moment Results}

In order to combine the three samples for which the magnetic moment measurement is relevant we used the master fitter as discussed in Section 4.6. This analysis gives a magnetic moment of

$$
\mu_{\Omega^{-}}=-2.024 \pm 0.056 \mu_{N}
$$

with a $\chi^{2}$ of $1 \times 10^{-3}$ for two degrees of freedom. The error given is purely statistical

based on a variation of $\chi^{2}$ by one. The error is consistent with that expected from the number of events and the polarization of the sample.

\subsubsection{Higher Order Precession Angles}

The precession angle and magnetic moment from a single field measurement, can only be determined to $\pm n \pi$ where $n$ is a positive integer. Thus the magnetic moment results presented above are the lowest order results. The best way to remove the uncertainty of $\pm n \pi$ in the precession angle is to fit the data using various field integrals to a single magnetic moment value by making a linear fit of precession angle versus precession field value constrained to have zero precession for zero field. Table 5.4 gives precession angle and $\int \mathrm{Bdl}$ data for this measurement and a previous measurement.

Table 5.5 shows $\chi^{2}$ (per degree of freedom) values for various $n$ values for a fit using the two field integrals from this experiment as well as those using measurements from a previous experiment. By using the data from Fermilab E756 we are able to conclude that the lowest order solution $(n=0)$ is indeed the correct solution. 

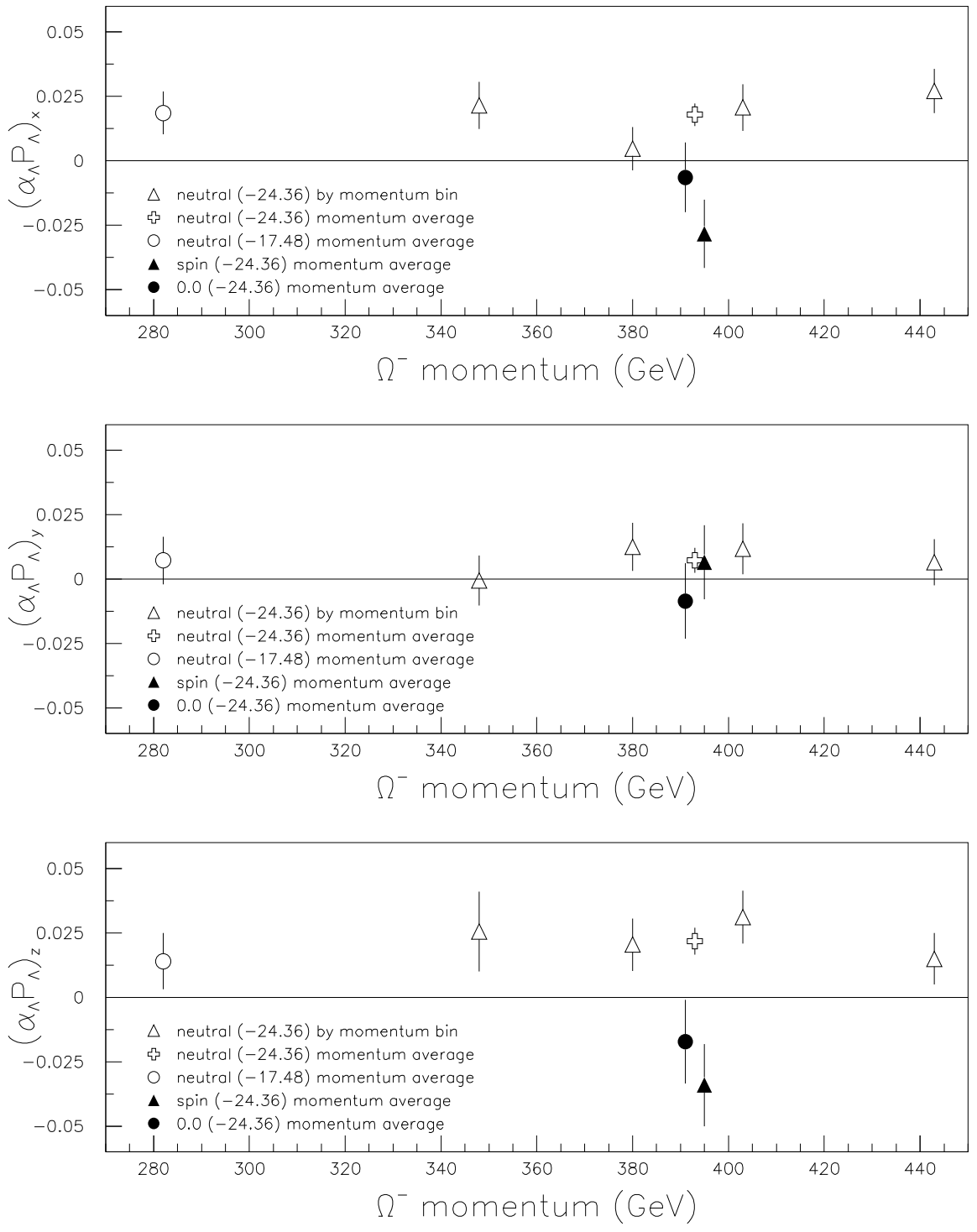

Figure 5.1: The polarization signals in $\mathrm{x}, \mathrm{y}, \mathrm{z}$ vs. momentum for the neutral production sample with $\int \mathrm{Bdl}=-24.36$ and the momentum averaged results for the four data samples analyzed using the hybrid monte carlo technique 

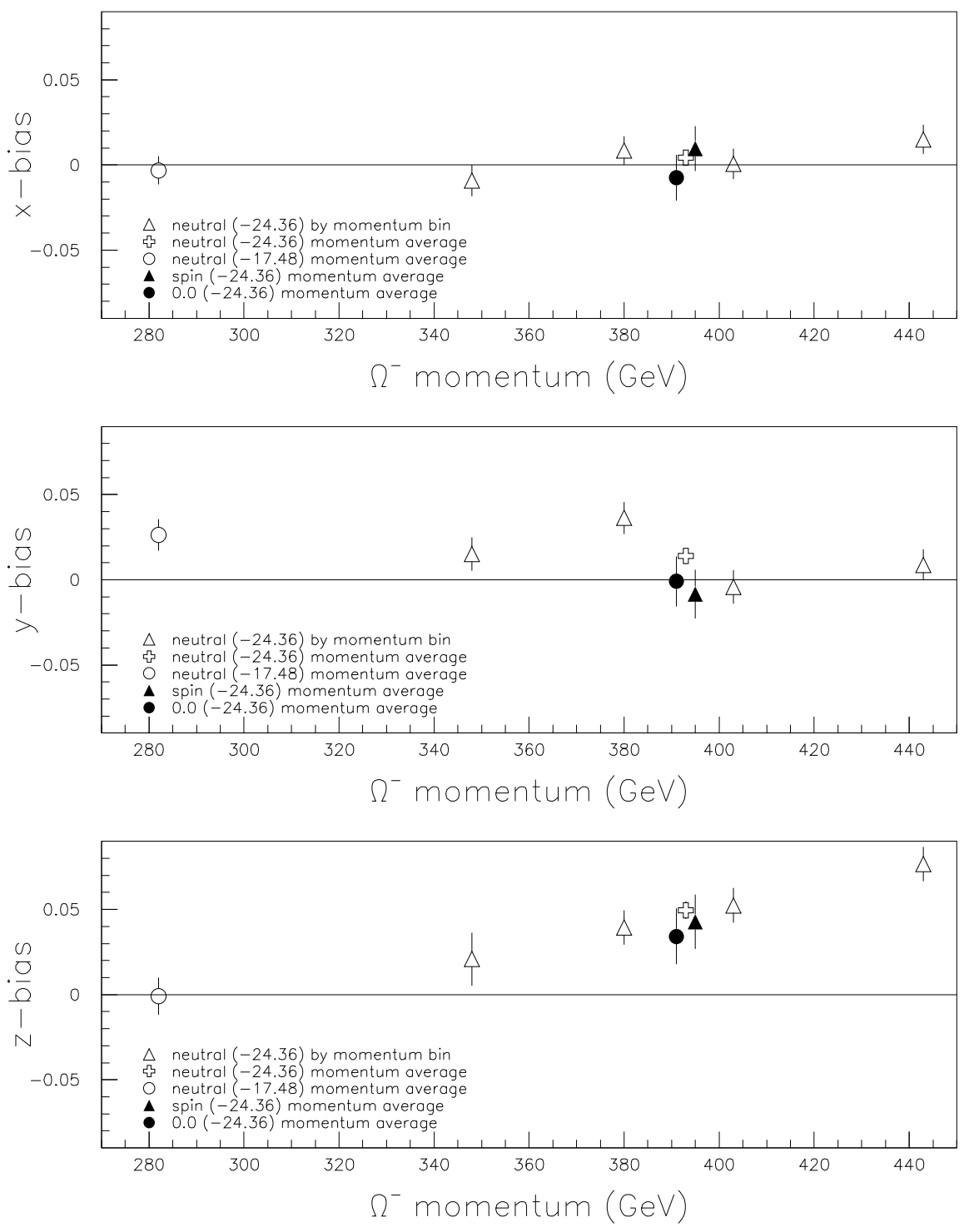

Figure 5.2: The biases in $\mathrm{x}, \mathrm{y}, \mathrm{z}$ vs. momentum for the neutral production sample with $\int \mathrm{Bdl}=-24.36$ and the momentum averaged results for the four data samples. 


\begin{tabular}{c|c|c} 
Experiment & $\int \mathrm{Bdl}(\mathrm{T} \cdot \mathrm{m})$ & $\Phi($ Radians $)$ \\
\hline E800 neutral & $-24.36 \pm 0.24$ & $0.88 \pm 0.17$ \\
E800 spin & $-24.36 \pm 0.24$ & $0.88 \pm 0.32$ \\
E800 neutral & $-17.48 \pm 0.17$ & $0.65 \pm 0.43$ \\
E756 & $-19.53 \pm 0.19$ & $0.58 \pm 0.42$ \\
E756 & $-14.77 \pm 0.15$ & $0.34 \pm 0.46$
\end{tabular}

Table 5.4: The field integrals $\int \mathrm{Bdl}$ and precession angles $(\Phi)$ for Fermilab E800 and Fermilab E756 $\Omega^{-}$magnetic moment measurements.

\begin{tabular}{|c|c|c|}
\hline $\begin{array}{c}n \\
(n \pi)\end{array}$ & $\begin{array}{c}\chi^{2} / \text { d.f. } \\
(\mathrm{E} 800 \text { data })\end{array}$ & $\begin{array}{c}\chi^{2} / \text { d.f. } \\
\text { (E800 and E756 data) }\end{array}$ \\
\hline 0 & 0.001 & 0.063 \\
\hline+1 & 2.08 & 2.38 \\
\hline-1 & 1.92 & 3.69 \\
\hline+2 & 8.18 & 10.92 \\
\hline-2 & 7.85 & 13.26 \\
\hline
\end{tabular}

Table 5.5: The $\chi^{2}$ per degree of freedom for the linear $\Phi$ vs $\int B d 1$ using E 800 data only and using E756 and E800 data.

Figures 5.3, and 5.4 show the data points and the best fit line for $\Phi$ vs $\int B d l$ for both cases.

\subsection{Systematic Studies}

In order to study systematic effects in the magnetic moment analysis we made use of the subsets of the $\Omega^{-}$data sample and monte carlo event samples.

The magnetic moment results were also determined from the ration of the $\mathrm{x}$ and $\mathrm{z}$ polarization components from each subsample of the data. Table 5.6, gives the lowest order precession angle, $\Phi$ for the neutral production data sample $\left(\int \mathrm{Bdl}=-24.36 \mathrm{~T} \cdot \mathrm{m}\right)$ for four momentum bins as well as the magnetic moment result $\mu_{\Omega^{-}}$for each momentum bin. The statistical errors for the measurements are given by

$$
\Delta \Phi=\left[\frac{P_{x}^{2}\left(\Delta P_{z}\right)^{2}+P_{z}^{2}\left(\Delta P_{x}\right)^{2}}{\left(P_{x}^{2}+P_{z}^{2}\right)^{2}}\right]^{1 / 2}
$$




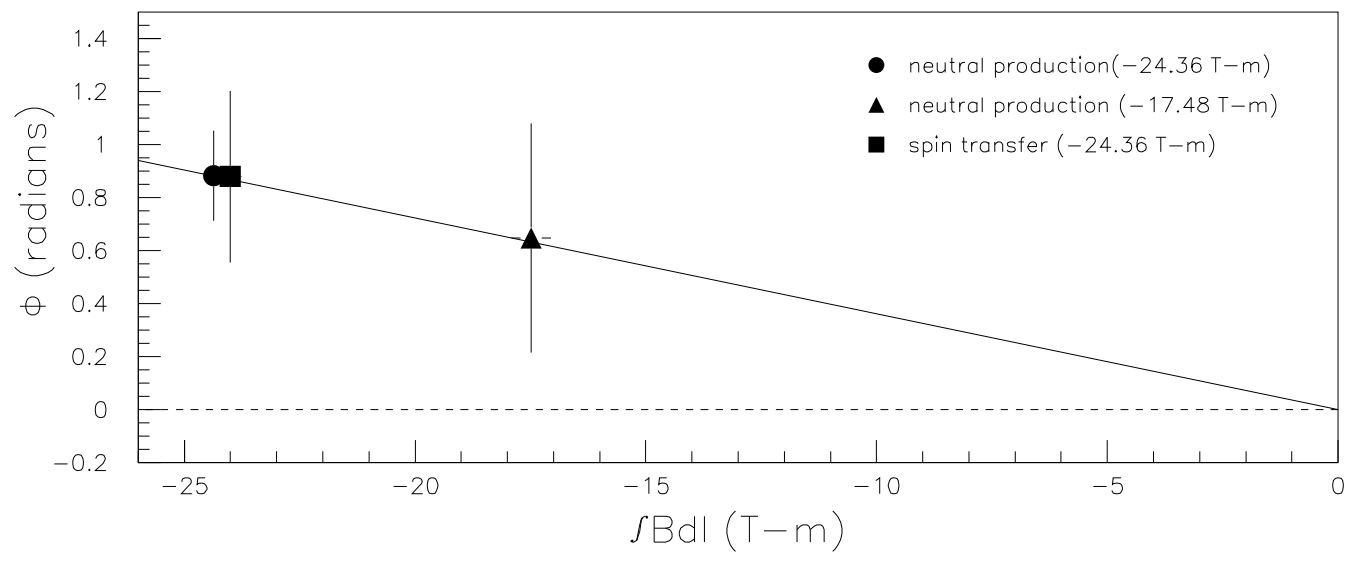

Figure 5.3: The precession angle $\Phi$ vs. the precession field $\left(\int \mathrm{Bdl}\right)$ for the three polarized data samples from this measurement. The best fit line (constrained through 0 ), which corresponds to the first order $(n=0)$ solution.

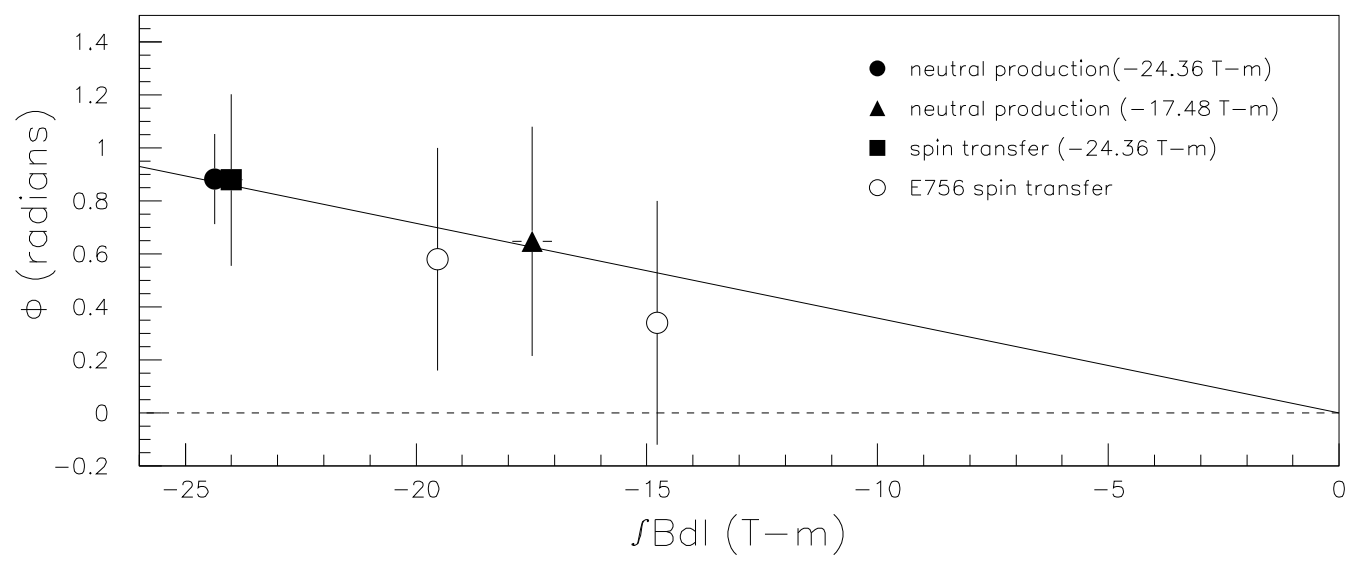

Figure 5.4: The precession angle $\Phi$ vs. the precession field $\left(\int \mathrm{Bdl}\right)$ for the three polarized data samples from this measurement and the two points from the previous measurement (E756). The best fit line, which corresponds to the first order $(\mathrm{n}=0)$ solution. 
with $\mathrm{P}_{x}$ and $\mathrm{P}_{z}$ the $\mathrm{x}$ and $\mathrm{z}$ polarization and their errors, $\Delta \mathrm{P}_{x}$ and $\Delta \mathrm{P}_{z}$, determined by the methods given in Chapter 4 .

The statistical error in the magnetic moment is given by

$$
\Delta \mu_{\Omega}=\frac{2 m_{p} c S_{\Omega}}{e \hbar} \frac{1}{\int B d l} \Delta \Phi
$$

where $m_{p}$ is the proton mass, $S_{\Omega}$ is the spin of the $\Omega^{-}$, and $\int B d l$ is the field integral in Tesla·meters.

\begin{tabular}{c|c|c}
$\begin{array}{c}\text { Mom. }(\mathrm{GeV} / \mathrm{c}) \\
10^{3} \text { Events }\end{array}$ & $\begin{array}{c}\Phi \\
\text { (radians) }\end{array}$ & $\begin{array}{c}\mu_{\Omega^{-}} \\
\left(\mu_{n}\right)\end{array}$ \\
\hline 348 & & \\
42.4 & $0.869 \pm 0.307$ & $-2.018 \pm 0.119$ \\
\hline 380 & & \\
42.5 & $1.341 \pm 0.407$ & $-2.200 \pm 0.157$ \\
\hline 403 & & \\
37.2 & $0.983 \pm 0.252$ & $-2.062 \pm 0.097$ \\
\hline 443 & & \\
44.3 & $0.501 \pm 0.311$ & $-1.878 \pm 0.120$ \\
\hline
\end{tabular}

Table 5.6: The precession angles $(\Phi)$ and $\Omega^{-}$magnetic moment results for the neutral production sample at $\int \mathrm{Bdl}=24.36 \mathrm{~T} \cdot \mathrm{m}$.

\begin{tabular}{c|c|c|c} 
Sample type & $\begin{array}{c}\text { Mom. }(\mathrm{GeV} / \mathrm{c}) \\
10^{3} \text { Events }\end{array}$ & $\begin{array}{c}\Phi \\
\text { (Radians) }\end{array}$ & $\begin{array}{c}\mu_{\Omega^{-}} \\
\left(\mu_{n}\right)\end{array}$ \\
\hline Neutral & 393 & & \\
Prod. (-24.36) & 166.5 & $0.881 \pm 0.169$ & $-2.023 \pm 0.065$ \\
\hline Neutral & 282 & & \\
Prod. (-17.48) & 50.2 & $0.647 \pm 0.432$ & $-2.031 \pm 0.232$ \\
\hline Spin-transfer & 393 & & \\
Prod (-24.36) & 18.3 & $0.879 \pm 0.324$ & $-2.022 \pm 0.125$ \\
\hline
\end{tabular}

Table 5.7: The momentum averaged measured precession angles $(\Phi)$ and $\Omega^{-}$magnetic moment results for the three production methods.

Figure 5.5 shows the relationship between the magnetic moment and the $\Omega^{-}$momentum for the four momentum bins of the neutral production data $\left(\int \mathrm{Bdl}=-24.36 \mathrm{~T} \cdot \mathrm{m}\right)$ and the momentum averaged results for all four data samples. The magnetic moment 
results for all subsamples of the data set agree to within statistical errors and give no indication of momentum dependence for the magnetic moment measurement.

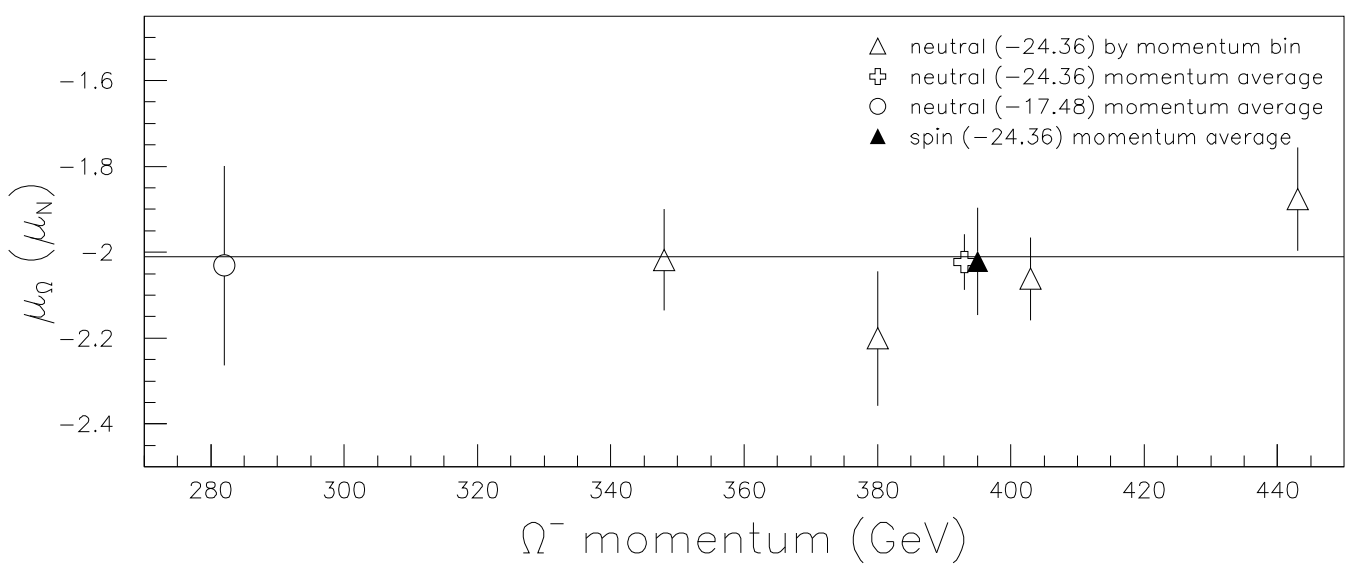

Figure 5.5: The magnetic moment results vs. momentum for the neutral production sample with $\int \mathrm{Bdl}=-24.36 \mathrm{~T} \cdot \mathrm{m}$ and the momentum averaged results for the four data samples.

\subsubsection{The Effect of the Selection Criteria}

The data was analyzed with tighter mass and $\chi^{2} /$ d.f. criteria to study the systematic effect of the reconstruction selection criteria. Table 5.8 shows the effect of tightening the mass criteria from $\pm 15 \mathrm{MeV}$ to $\pm 7.5 \mathrm{MeV}$ on the momentum averaged results for the four $\Omega^{-}$data samples, as well as the master fit result. Table 5.9 shows the effect of tightening the $\chi_{G}^{2} /$ d.f. criteria from 4.0 to 3.0 and the $\chi_{K}^{2}$ criteria from 15.0 to 10.0 . The overall master fit results for each of the three cases are shown in Table 5.10.

Comparison of Tables 5.8 and 5.9 with the polarization and magnetic moment results given in Sections 5.1 and 5.2 show that the variation of the results with the changes in selection criteria are less than the $1 \sigma$ statistical errors of the individual points. The master fit results shown in Table 5.10 vary by about one half of a standard deviation. 


\begin{tabular}{c|c|c|c|c|c}
$\begin{array}{c}\text { Sample } \\
\text { type }\end{array}$ & $\begin{array}{c}\text { Mom. }(\mathrm{GeV} / \mathrm{c}) \\
10^{3} \text { Events }\end{array}$ & View & $\begin{array}{c}\text { Polarization } \\
\alpha_{\Lambda} \mathrm{P}_{\Lambda}\end{array}$ & $\begin{array}{c}\Phi \\
\text { radians }\end{array}$ & $\begin{array}{c}\mu_{\Omega^{-}} \\
\mu_{N}\end{array}$ \\
\hline $\begin{array}{c}\text { neutral } \\
\text { prod. }\end{array}$ & 352 & $\mathrm{x}$ & $0.0191 \pm 0.0098$ & & $-2.051 \pm 0.125$ \\
$(-24.36)$ & 37.9 & $\mathrm{y}$ & $0.0028 \pm 0.0103$ & $0.955 \pm 0.323$ & \\
\hline $\begin{array}{c}\text { neutral } \\
\text { prod. }\end{array}$ & 380 & $\mathrm{x}$ & $0.0075 \pm 0.0088$ & & \\
$(-24.36)$ & 38.6 & $\mathrm{y}$ & $0.0124 \pm 0.0099$ & $1.157 \pm 0.489$ & $-2.130 \pm 0.189$ \\
\hline neutral & 403 & $\mathrm{x}$ & $0.0171 \pm 0.0106$ & & \\
prod. & 34.2 & $\mathrm{y}$ & $0.0155 \pm 0.0103$ & $1.092 \pm 0.250$ & $-2.105 \pm 0.096$ \\
$(-24.36)$ & & $\mathrm{z}$ & $0.0345 \pm 0.0108$ & & \\
\hline neutral & 438 & $\mathrm{x}$ & $0.0268 \pm 0.0090$ & & \\
prod. & 40.3 & $\mathrm{y}$ & $0.0015 \pm 0.0095$ & $0.588 \pm 0.310$ & $-1.910 \pm 0.120$ \\
$(-24.36)$ & & $\mathrm{z}$ & $0.0179 \pm 0.0104$ & & \\
\hline neutral & 393 & $\mathrm{x}$ & $0.0174 \pm 0.0046$ & & $-2.039 \pm 0.067$ \\
average & 151.0 & $\mathrm{y}$ & $0.0076 \pm 0.0050$ & $0.9219 \pm 0.173$ & \\
$(-24.36)$ & & $\mathrm{z}$ & $0.0230 \pm 0.0055$ & & \\
\hline neutral & 282 & $\mathrm{x}$ & $0.0207 \pm 0.0086$ & & \\
prod. & 36.8 & $\mathrm{y}$ & $0.0076 \pm 0.0050$ & $0.6822 \pm 0.388$ & $-2.050 \pm 0.209$ \\
$(-17.48)$ & & $\mathrm{z}$ & $0.0230 \pm 0.0055$ & & \\
\hline spin & 393 & $\mathrm{x}$ & $-0.0228 \pm 0.0137$ & & \\
transfer & 17.0 & $\mathrm{y}$ & $0.0040 \pm 0.0149$ & $0.988 \pm 0.352$ & $-2.065 \pm 0.136$ \\
$(-24.36)$ & & $\mathrm{z}$ & $-0.0346 \pm 0.0165$ & & \\
\hline $0.0 \mathrm{mr}$ & 393 & $\mathrm{x}$ & $-0.0016 \pm 0.0144$ & & \\
& 17.0 & $\mathrm{y}$ & $-0.0079 \pm 0.0155$ & & \\
$(-24.36)$ & & $\mathrm{z}$ & $-0.0208 \pm 0.0172$ & & \\
\hline & & & & & \\
\hline
\end{tabular}

Table 5.8: The measured $\Omega^{-}$polarizations, precession angles, and magnetic moment for four momentum bins of neutral production sample with $\int \mathrm{Bdl}=24.36 \mathrm{~T} \cdot \mathrm{m}$ and momentum averaged results for the four data data samples. This analysis used the tight mass cuts at $\pm 7.5 \mathrm{MeV}$. 


\begin{tabular}{c|c|c|c|c|c}
$\begin{array}{c}\text { Sample } \\
\text { type }\end{array}$ & $\begin{array}{c}\text { Mom. }(\mathrm{GeV} / \mathrm{c}) \\
10^{3} \text { Events }\end{array}$ & View & $\begin{array}{c}\text { Polarization } \\
\alpha_{\Lambda} \mathrm{P}_{\Lambda}\end{array}$ & $\begin{array}{c}\Phi \\
\text { radians }\end{array}$ & $\begin{array}{c}\mu_{\Omega^{-}} \\
\mu_{N}\end{array}$ \\
\hline $\begin{array}{c}\text { neutral } \\
\text { prod. }\end{array}$ & 352 & $\mathrm{x}$ & $0.0206 \pm 0.0095$ & & $-2.063 \pm 0.108$ \\
$(-24.36)$ & 39.4 & $\mathrm{y}$ & $0.0031 \pm 0.0100$ & $0.985 \pm 0.280$ & \\
\hline neutral & 380 & $\mathrm{X}$ & $0.0078 \pm 0.0087$ & & \\
prod. & 39.6 & $\mathrm{y}$ & $0.0102 \pm 0.0097$ & $1.194 \pm 0.424$ & $-2.144 \pm 0.164$ \\
$(-24.36)$ & & $\mathrm{z}$ & $0.0198 \pm 0.0106$ & & \\
\hline neutral & 403 & $\mathrm{x}$ & $0.0207 \pm 0.0093$ & & $-2.058 \pm 0.103$ \\
prod. & 34.7 & $\mathrm{y}$ & $0.0179 \pm 0.0102$ & $0.970 \pm 0.267$ & \\
$(-24.36)$ & & $\mathrm{z}$ & $0.0303 \pm 0.0108$ & & $-1.883 \pm 0.107$ \\
\hline neutral & 438 & $\mathrm{x}$ & $0.0304 \pm 0.0089$ & & \\
prod. & 40.4 & $\mathrm{y}$ & $0.0016 \pm 0.0093$ & $0.517 \pm 0.277$ & \\
$(-24.36)$ & & $\mathrm{z}$ & $0.0179 \pm 0.0104$ & & $-2.020 \pm 0.063$ \\
\hline neutral & 393 & $\mathrm{x}$ & $0.0196 \pm 0.0046$ & & \\
average & 155.0 & $\mathrm{y}$ & $0.0075 \pm 0.0049$ & $0.874 \pm 0.163$ & \\
$(-24.36)$ & & $\mathrm{z}$ & $0.0234 \pm 0.0055$ & & \\
\hline neutral & 282 & $\mathrm{x}$ & $0.0204 \pm 0.0086$ & & \\
prod. & 46.0 & $\mathrm{y}$ & $0.0048 \pm 0.0097$ & $0.8312 \pm 0.333$ & $-2.130 \pm 0.179$ \\
$(-17.48)$ & & $\mathrm{z}$ & $0.0223 \pm 0.0116$ & & \\
\hline spin & 393 & $\mathrm{x}$ & $-0.0286 \pm 0.0137$ & & \\
transfer & 16.9 & $\mathrm{y}$ & $0.0079 \pm 0.0149$ & $0.873 \pm 0.336$ & $-2.020 \pm 0.130$ \\
$(-24.36)$ & & $\mathrm{z}$ & $-0.0341 \pm 0.0166$ & & \\
\hline $0.0 \mathrm{mr}$ & 393 & $\mathrm{x}$ & $-0.0031 \pm 0.0140$ & & \\
& 16.3 & $\mathrm{y}$ & $-0.0071 \pm 0.0151$ & & \\
$(-24.36)$ & & $\mathrm{z}$ & $-0.0177 \pm 0.0171$ & & \\
\hline
\end{tabular}

Table 5.9: The measured $\Omega^{-}$polarizations, precession angles, and magnetic moment for four momentum bins of neutral production sample with $\int \mathrm{Bdl}=24.36 \mathrm{~T} \cdot \mathrm{m}$ and momentum averaged results for the four data data samples. This analysis used the tight $\chi_{G}^{2} /$ d.f. cuts at $3.0 /$ d.f. and $\chi_{K}^{2} 10.0$

\begin{tabular}{c|c|c|c|c} 
sample & $\chi_{G}^{2} /$ d.f. & $\chi_{K}^{2}$ & mass cut $(\mathrm{Mev})$ & $\mu_{\Omega}-\left(\mu_{N}\right)$ \\
\hline Normal & 4.0 & 15.0 & \pm 15.0 & $-2.024 \pm 0.056$ \\
Tight mass & 4.0 & 15.0 & \pm 7.5 & $-2.044 \pm 0.057$ \\
Tight $\chi_{K}^{2}$ and $\chi_{G}^{2} /$ d.f. & 3.0 & 10.0 & \pm 15.0 & $-2.028 \pm 0.054$
\end{tabular}

Table 5.10: The master fit results for the analysis using three selection criteria. 


\subsubsection{Sample Contamination}

The selection criteria for the $\Omega^{-}$sample were discussed in Chapter 3 . Several methods were used to study the effect of the two major backgrounds on the magnetic moment and polarization results. The tightened mass selection criteria reduced both the $\Xi^{-} \rightarrow \Lambda \pi^{-}$ and $\Omega^{-} \rightarrow \Xi^{0} \pi^{-}$backgrounds by a factor of two. The result of this study, shown in Tables 5.8, and 5.10 indicates that a reduction of these backgrounds did not effect the measurement.

To further study the effect of the $\Xi^{-}$background we allowed a fraction of $\Xi^{-} \mathrm{s}$ to "bleed-through" into the data sample. By randomly allowing 0.003 of the $\Xi^{-}$events through the kinematic angle cut a background level of $2.5 \%$ was added to the data sample. The results from analyzing this polluted data sample are shown in Table 5.11.

Since the level of $\Omega^{-} \rightarrow \Xi^{0} \pi^{-}$in the data sample cannot be increased significantly by the technique used to study the $\Xi^{-}$background, it was necessary to create a monte carlo sample of $\Omega^{-} \rightarrow \Xi^{0} \pi^{-}$events. The generated background events were combined with a sample of monte carlo generated $\Omega^{-} \rightarrow \Lambda \mathrm{K}^{-}$roughly 3.3 times larger than the data sample under analysis for this measurement. Table 5.12 shows the input polarization, the output polarization, and the calculated magnetic moment for various levels of pollution by this background.

The worst case estimate for total contamination of the $\Omega^{-}$sample gives a pollution level of about $3 \%$. These studies indicate that the results of this analysis are uneffected by contamination levels at least four times larger than those present in the data sample.

\subsection{Internal Checks}

\subsubsection{Consistancy Checks}

There are several internal checks available to us in this analysis. For some subset of the data, spin transfer and $0.0 \mathrm{mr}$ production, the beam is in the same place in the 


\begin{tabular}{|c|c|c|c|c|c|}
\hline $\begin{array}{l}\text { Sample } \\
\text { type }\end{array}$ & $\begin{array}{c}\text { Mom. }(\mathrm{GeV} / \mathrm{c}) \\
10^{3} \text { Events }\end{array}$ & View & $\begin{array}{c}\text { Polarization } \\
\qquad \alpha_{\Lambda} \mathrm{P}_{\Lambda}\end{array}$ & $\begin{array}{c}\Phi \\
\text { radians }\end{array}$ & $\begin{array}{c}\mu_{\Omega^{-}} \\
\mu_{N}\end{array}$ \\
\hline $\begin{array}{c}\text { neutral } \\
\text { prod. } \\
(-24.36)\end{array}$ & $\begin{array}{l}352 \\
43.6\end{array}$ & $\begin{array}{l}\mathrm{x} \\
\mathrm{y} \\
\mathrm{z}\end{array}$ & $\begin{array}{l}0.0224 \pm 0.0091 \\
0.0065 \pm 0.0096 \\
0.0244 \pm 0.0114\end{array}$ & $0.828 \pm 0.310$ & $-2.003 \pm 0.120$ \\
\hline $\begin{array}{c}\text { neutral } \\
\text { prod. } \\
(-24.36)\end{array}$ & $\begin{array}{l}380 \\
43.5\end{array}$ & $\begin{array}{l}x \\
y \\
z\end{array}$ & $\begin{array}{l}0.0077 \pm 0.0083 \\
0.0097 \pm 0.0096 \\
0.0179 \pm 0.0099\end{array}$ & $1.165 \pm 0.443$ & $-2.133 \pm 0.171$ \\
\hline $\begin{array}{c}\text { neutral } \\
\text { prod. } \\
(-24.36)\end{array}$ & $\begin{array}{l}403 \\
38.3\end{array}$ & $\begin{array}{l}x \\
y \\
z\end{array}$ & $\begin{array}{l}0.0199 \pm 0.0085 \\
0.0086 \pm 0.0098 \\
0.0285 \pm 0.0102\end{array}$ & $0.962 \pm 0.269$ & $-2.054 \pm 0.104$ \\
\hline $\begin{array}{c}\text { neutral } \\
\text { prod. } \\
(-24.36)\end{array}$ & $\begin{array}{l}438 \\
44.4\end{array}$ & $\begin{array}{l}x \\
y \\
z\end{array}$ & $\begin{array}{l}0.0245 \pm 0.0085 \\
0.0054 \pm 0.0089 \\
0.0148 \pm 0.0099\end{array}$ & $0.544 \pm 0.333$ & $-1.893 \pm 0.129$ \\
\hline $\begin{array}{l}\text { neutral } \\
\text { average } \\
(-24.36)\end{array}$ & $\begin{array}{c}393 \\
170.7\end{array}$ & $\begin{array}{l}x \\
y \\
z\end{array}$ & $\begin{array}{l}0.0181 \pm 0.0044 \\
0.0073 \pm 0.0047 \\
0.0203 \pm 0.0052\end{array}$ & $0.843 \pm 0.175$ & $-2.008 \pm 0.067$ \\
\hline $\begin{array}{c}\text { neutral } \\
\text { prod. } \\
(-17.48)\end{array}$ & $\begin{array}{l}282 \\
51.8\end{array}$ & $\begin{array}{l}x \\
y \\
z\end{array}$ & $\begin{array}{l}0.0155 \pm 0.0082 \\
0.0022 \pm 0.0092 \\
0.0130 \pm 0.0107\end{array}$ & $0.700 \pm 0.481$ & $-2.06 \pm 0.258$ \\
\hline $\begin{array}{c}\text { spin } \\
\text { transfer } \\
(-24.36) \\
\end{array}$ & $\begin{array}{c}393 \\
18.7\end{array}$ & $\begin{array}{l}\mathrm{x} \\
\mathrm{y} \\
\mathrm{z}\end{array}$ & $\begin{array}{c}-0.0268 \pm 0.0130 \\
0.0139 \pm 0.0142 \\
-0.0324 \pm 0.0157\end{array}$ & $0.881 \pm 0.337$ & $-2.023 \pm 0.130$ \\
\hline $\begin{array}{c}0.0 \mathrm{mr} \\
(-24.36)\end{array}$ & $\begin{array}{c}393 \\
18.0\end{array}$ & $\begin{array}{l}x \\
y \\
z\end{array}$ & $\begin{array}{l}-0.0052 \pm 0.0134 \\
-0.0109 \pm 0.0144 \\
-0.0110 \pm 0.0160\end{array}$ & -- & - \\
\hline
\end{tabular}

Table 5.11: The measured $\Omega^{-}$polarizations, precession angles, and magnetic moment for four momentum bins of neutral production sample with $\int \mathrm{Bdl}=24.36 \mathrm{~T} \cdot \mathrm{m}$ and momentum averaged results for the four data data samples. These samples have $2.5 \%$ $\Xi^{-}$events included in the sample. 


\begin{tabular}{c|c|c|c|c|c} 
& & $\mathrm{m} c$ & \multicolumn{3}{|c}{ Level of $\Omega^{-} \rightarrow \Xi^{0} \pi^{-}$contamination } \\
mom. & view & input & 0.00 & 0.043 & 0.129 \\
$(\mathrm{GeV} / \mathrm{c})$ & $\mu$ & $\alpha_{\Lambda} \mathrm{P}_{\Lambda}$ & $\alpha_{\Lambda} \mathrm{P}_{\Lambda}$ & $\alpha_{\Lambda} \mathrm{P}_{\Lambda}$ & $\alpha_{\Lambda} \mathrm{P}_{\Lambda}$ \\
\hline \multirow{3}{*}{352} & $\mathrm{x}$ & 0.016 & $0.028 \pm 0.005$ & $0.023 \pm 0.005$ & $0.019 \pm 0.005$ \\
& $\mathrm{y}$ & 0.000 & $-0.003 \pm 0.006$ & $-0.002 \pm 0.005$ & $0.001 \pm 0.005$ \\
& $\mathrm{z}$ & 0.023 & $0.027 \pm 0.006$ & $0.027 \pm 0.006$ & $0.021 \pm 0.006$ \\
& $\mu$ & -2.050 & $-2.015 \pm 0.063$ & $-2.019 \pm 0.061$ & $-2.005 \pm 0.071$ \\
\hline \multirow{3}{*}{380} & $\mathrm{x}$ & 0.016 & $0.019 \pm 0.005$ & $0.018 \pm 0.005$ & $0.017 \pm 0.004$ \\
& $\mathrm{y}$ & 0.000 & $0.002 \pm 0.005$ & $0.001 \pm 0.005$ & $0.001 \pm 0.005$ \\
& $\mathrm{z}$ & 0.023 & $0.027 \pm 0.006$ & $0.026 \pm 0.005$ & $0.024 \pm 0.005$ \\
& $\mu$ & -2.050 & $-2.054 \pm 0.057$ & $-2.052 \pm 0.060$ & $-2.051 \pm 0.061$ \\
\hline \multirow{3}{*}{403} & $\mathrm{x}$ & 0.016 & $0.014 \pm 0.005$ & $0.015 \pm 0.005$ & $0.015 \pm 0.005$ \\
& $\mathrm{y}$ & 0.000 & $0.008 \pm 0.006$ & $0.008 \pm 0.006$ & $0.009 \pm 0.006$ \\
& $\mathrm{z}$ & 0.023 & $0.024 \pm 0.006$ & $0.023 \pm 0.006$ & $0.022 \pm 0.006$ \\
& $\mu$ & -2.050 & $-2.083 \pm 0.083$ & $-2.072 \pm 0.074$ & $-2.055 \pm 0.075$ \\
\hline \multirow{3}{*}{438} & $\mathrm{x}$ & 0.016 & $0.015 \pm 0.005$ & $0.015 \pm 0.005$ & $0.014 \pm 0.005$ \\
& $\mathrm{y}$ & 0.000 & $0.007 \pm 0.005$ & $0.006 \pm 0.005$ & $0.007 \pm 0.005$ \\
& $\mathrm{z}$ & 0.023 & $0.018 \pm 0.005$ & $0.018 \pm 0.005$ & $0.017 \pm 0.005$ \\
& $\mu$ & -2.050 & $-2.024 \pm 0.083$ & $-2.017 \pm 0.083$ & $-2.022 \pm 0.086$ \\
\hline \multirow{3}{*}{ Avg. } & $\mathrm{x}$ & 0.016 & $0.018 \pm 0.002$ & $0.018 \pm 0.002$ & $0.017 \pm 0.002$ \\
393 & $\mathrm{y}$ & 0.000 & $0.003 \pm 0.003$ & $0.003 \pm 0.003$ & $0.004 \pm 0.003$ \\
& $\mathrm{z}$ & 0.023 & $0.024 \pm 0.003$ & $0.023 \pm 0.003$ & $0.021 \pm 0.003$ \\
& $\mu$ & -2.050 & $-2.040 \pm 0.035$ & $-2.036 \pm 0.034$ & $-2.031 \pm 0.037$ \\
\hline \multirow{3}{*}{3} & & & & \multicolumn{3}{|c}{}
\end{tabular}

Table 5.12: The input and analyzed polarizations for monte carlo $\Omega^{-}$neutral production events with increasing levels of $\Omega^{-} \rightarrow \Xi^{0} \pi^{-}$pollution. 
apparatus for all data samples. Under these conditions we could analyze the data under the assuption of acceptance cancellation for the positive and negative production angles. The acceptance cancellation analysis allows a check of the $\Omega^{-}$polarization and magnetic moment results by direct comparison without the intervention of the hybrid monte carlo.

Further we observed in Chapter 1 that a non-zero y component of the polarization violates parity symmetry in the strong interaction since the polarization would not be perpendicular to the production plane. The hybrid monte carlo results presented in Section 5.1.1 show no significant polarization in the y view. There is also no significant polarization in the $0.0 \mathrm{mr}$ data sample. Such a signal could indicate a false signal or systematic error in the data.

As another check we made a high precision measurement of $\Xi^{-}$target polarization and magnetic moment, which allowed us to compare those results to the existing results for those quantities.

\subsubsection{Acceptance Cancellation Results}

The four data samples were also analyzed using the acceptance cancellation technique as described in Chapter 4. While this technique is not as robust with respect to mismatches between positive and negative angle acceptance as the hybrid monte carlo method it is a complementary analysis and useful for comparison. Table 5.13, gives the results for this analysis on the neutral production sample $\left(\int \mathrm{Bdl}=-24.36 \mathrm{~T} \cdot \mathrm{m}\right)$. Table 5.14 give the momentum averaged results for all four samples.

There are some interesting features in the acceptance cancellation results. First there is a y polarization signal for the neutral data sample, while for the spin transfer and $0.0 \mathrm{mr}$ data there is none. This is a demonstration of the shortcomings of this type of analysis. The neutral beam mode probes different spectrometer regions for positive and negative production angles, while the location of the beam is fairly constant for 


\begin{tabular}{c|c|c|c|c}
$\begin{array}{c}\text { Mom. }(\mathrm{GeV} / \mathrm{c}) \\
10^{3} \text { Events }\end{array}$ & View & $\begin{array}{c}\text { Polarization } \\
\alpha_{\Lambda} \mathrm{P}_{\Lambda}\end{array}$ & $\begin{array}{c}\Phi \\
\text { (radians) }\end{array}$ & $\begin{array}{c}\mu_{\Omega^{-}} \\
\mu_{N}\end{array}$ \\
\hline 352 & $\mathrm{x}$ & $0.0224 \pm 0.0067$ & & \\
66.1 & $\mathrm{y}$ & $0.0261 \pm 0.0065$ & $0.686 \pm 0.0124$ & $-1.948 \pm 0.094$ \\
& $\mathrm{z}$ & $0.0183 \pm 0.0073$ & & \\
\hline 380 & $\mathrm{x}$ & $0.0169 \pm 0.0068$ & & \\
59.6 & $\mathrm{y}$ & $0.0380 \pm 0.0072$ & $0.850 \pm 0.275$ & $-2.011 \pm 0.106$ \\
& $\mathrm{z}$ & $0.0192 \pm 0.0073$ & & \\
\hline 403 & $\mathrm{x}$ & $0.0214 \pm 0.0074$ & & \\
52.4 & $\mathrm{y}$ & $0.0396 \pm 0.0076$ & $0.879 \pm 0.224$ & $-2.022 \pm 0.086$ \\
& $\mathrm{z}$ & $0.0259 \pm 0.0077$ & & \\
\hline 438 & $\mathrm{x}$ & $0.0320 \pm 0.0064$ & & $-1.849 \pm 0.074$ \\
68.9 & $\mathrm{y}$ & $0.0266 \pm 0.0066$ & $0.429 \pm 0.191$ & \\
\hline & $\mathrm{z}$ & $0.0146 \pm 0.0068$ & &
\end{tabular}

Table 5.13: The measured $\Omega^{-}$polarizations, precession angle and magnetic moment for the neutral production sample with $\int \mathrm{Bdl}=24.36 \pm 0.24 \mathrm{~T} \cdot \mathrm{m}$ using the acceptance cancellation method.

\begin{tabular}{c|c|c|c|c|c}
$\begin{array}{c}\text { Sample } \\
\text { type }\end{array}$ & $\begin{array}{c}\text { Mom. }(\mathrm{GeV} / \mathrm{c}) \\
10^{3} \text { Events }\end{array}$ & View & $\begin{array}{c}\text { Polarization } \\
\alpha_{\Lambda} \mathrm{P}_{\Lambda}\end{array}$ & $\begin{array}{c}\Phi \\
\text { (radians) }\end{array}$ & $\begin{array}{c}\mu_{\Omega-} \\
\mu_{N}\end{array}$ \\
\hline $\begin{array}{c}\text { Neutral } \\
\text { Prod. }\end{array}$ & 392 & $\mathrm{x}$ & $0.0224 \pm 0.0034$ & & \\
$(-24.36)$ & 247.1 & $\mathrm{y}$ & $0.0320 \pm 0.0035$ & $0.659 \pm 0.124$ & $-1.937 \pm 0.048$ \\
\hline Neutral & 282 & $\mathrm{x}$ & $0.0149 \pm 0.0058$ & & \\
Prod. & 82.5 & $\mathrm{y}$ & $0.0237 \pm 0.0061$ & $0.600 \pm 0.348$ & $-2.006 \pm 0.187$ \\
$(-17.48)$ & & $\mathrm{z}$ & $0.010 \pm 0.0065$ & & \\
\hline Spin & 393 & $\mathrm{x}$ & $-0.0264 \pm 0.0104$ & & \\
Trans. & 26.8 & $\mathrm{y}$ & $0.0015 \pm 0.0105$ & $0.938 \pm 0.237$ & $-2.142 \pm 0.092$ \\
$(-24.36)$ & & $\mathrm{z}$ & $-0.0360 \pm 0.0109$ & & \\
\hline $0.0 \mathrm{~m} . \mathrm{r}$ & 393 & $\mathrm{x}$ & $-0.0022 \pm 0.0105$ & & - \\
& 33.2 & $\mathrm{y}$ & $-0.0008 \pm 0.0107$ & - & - \\
$(-24.36)$ & & $\mathrm{z}$ & $-0.0178 \pm 0.0112$ & & \\
\hline
\end{tabular}

Table 5.14: The momentum averaged measured $\Omega^{-}$polarizations, precession angles, and magnetic moments for the four data samples analyzed using the acceptance cancellation method. 
both targeting angles in spin transfer mode, and is unchanged for $0.0 \mathrm{mr}$ production. We can conclude that the y polarization signal in the neutral production data sets is a false signal introduced by non-cancellation of the spectrometer acceptance.

For the spin-transfer and $0.0 \mathrm{mr}$ samples the polarization and magnetic moment results are in good agreement with those of the hybrid monte carlo analysis. Figure 5.6 shows the asymmetry histograms for the $\mathrm{x}, \mathrm{y}$, and $\mathrm{z}$ polarization components for the neutral production sample at $\int \mathrm{Bdl}=-24.36 \mathrm{~T} \cdot \mathrm{m}$, the lines on the plot are the asymmtries resulting from the hybrid monte carlo analysis. However, the momentum average result for neutral production for $\mu_{\Omega^{-}}$is about $1.3 \sigma$ lower than the hybrid monte carlo result. Again this is probably caused by the different beam positions for positive and negative production angle in this data set. The precession angle and the $\mathrm{x}$ and $\mathrm{z}$ polarization results are all within $1 \sigma$ of the hybrid results. In this case the acceptance cancellation $\mathrm{z}$ polarization is almost $1 \sigma$ low and the $\mathrm{x}$ polarization is almost $1 \sigma$ high producing a large error in the magnetic moment result.

\subsubsection{Cascade Minus Results}

In order to check the systematic effects in the data we made use of not only the $\Omega^{-}$ sample but also the $\Xi^{-}$sample. Using the more numerous $\Xi^{-}$events it is possible to make many subsets of the data with statistics comparable to the entire $\Omega^{-}$data set. The analysis of these subsets tests systematic effects within the data to higher precision than is available by studying the $\Omega^{-}$sample alone. Table 5.15 and 5.16 give the $\Xi^{-}$ polarizations for four momentum bins for $-24.36 \mathrm{~T} \cdot \mathrm{m}$ neutral and spin transfer production methods. The two results presented compare an analysis with and without the kinematic angle criteria see section 3.5. This allows a study of the systematic effect of this selection criteria on a sample that has very small background levels. The neutral production $\Xi^{-}$sample is unpolarized while the spin transfer production sample is polarized at the $15 \sigma$ to $20 \sigma$ level. Both of these samples are unaffected by the kinematic 

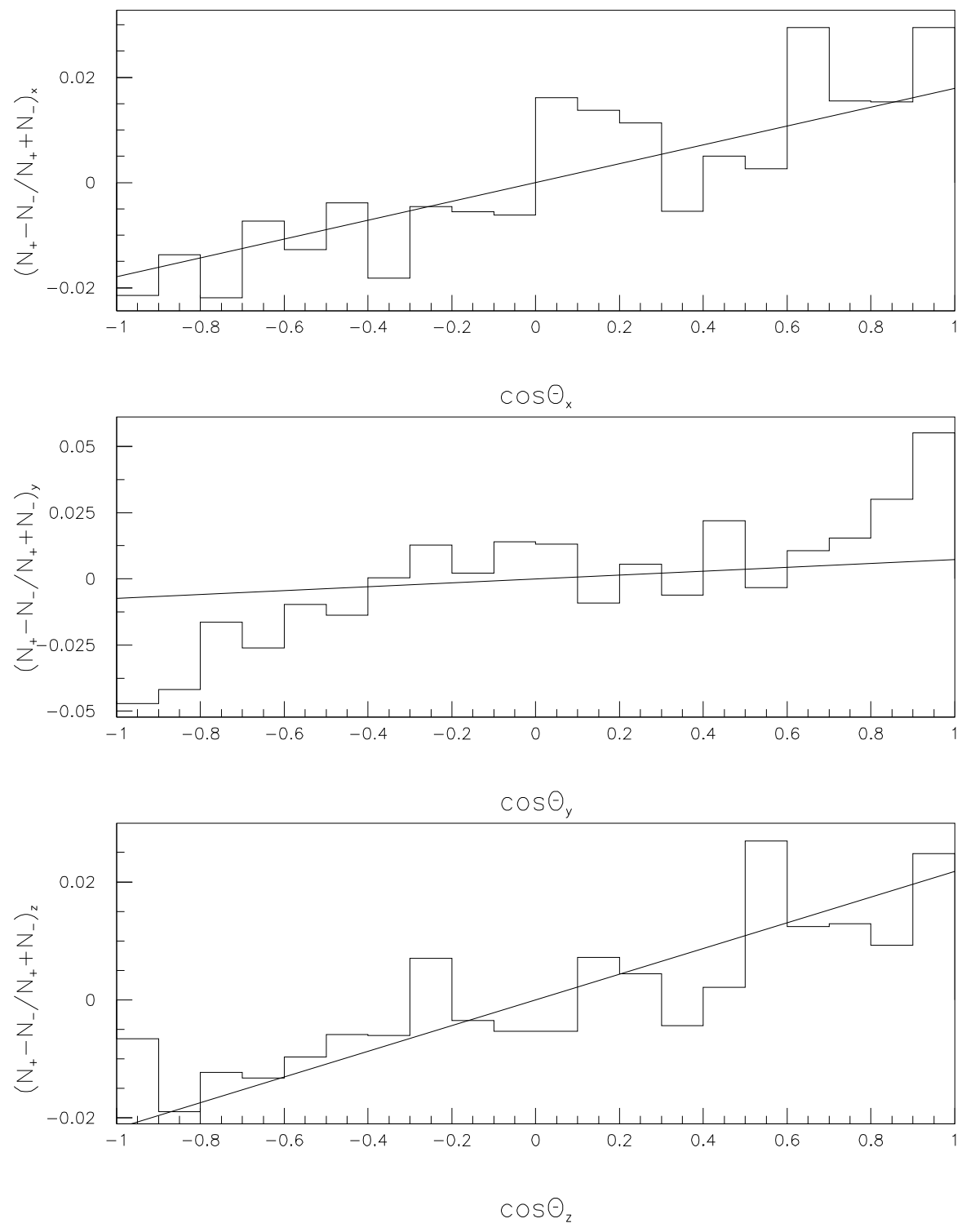

Figure 5.6: The $\mathrm{x}, \mathrm{y}$, and $\mathrm{z}$ asymmetry histograms for the neutral production $\Omega^{-}$sample at $\int \mathrm{Bdl}=-24.36 \mathrm{~T} \cdot \mathrm{m}$. The lines show the asymmetry result from the hybrid monte carlo analysis. 


\begin{tabular}{c|c|c|c} 
Mom. $(\mathrm{GeV} / \mathrm{c})$ & View & $\cos \theta_{K}$ vs. $\Phi$ & $\operatorname{no} \cos \theta_{K}$ vs. $\Phi$ \\
$10^{3}$ Events & $\mu_{\Xi-}\left(\mu_{N}\right)$ & $\alpha_{\Lambda} \mathrm{P}_{\Lambda}$ & $\alpha_{\Lambda} \mathrm{P}_{\Lambda}$ \\
\hline 352 & $\mathrm{x}$ & $0.0077 \pm 0.0040$ & $0.0097 \pm 0.0042$ \\
225.4 & $\mathrm{y}$ & $0.0099 \pm 0.0043$ & $0.0071 \pm 0.0045$ \\
212.0 & $\mathrm{Z}$ & $-0.0043 \pm 0.0048$ & $-0.0064 \pm 0.0050$ \\
& $\mu_{\Xi-}$ & $-0.643 \pm 0.020$ & $-0.656 \pm 0.018$ \\
\hline 380 & $\mathrm{x}$ & $-0.0038 \pm 0.0038$ & $-0.0056 \pm 0.0040$ \\
262.3 & $\mathrm{y}$ & $0.0010 \pm 0.0039$ & $0.0034 \pm 0.0041$ \\
241.8 & $\mathrm{z}$ & $-0.0073 \pm 0.0044$ & $-0.0061 \pm 0.0046$ \\
& $\mu_{\Xi-}$ & $-0.629 \pm 0.010$ & $-0.627 \pm 0.010$ \\
\hline 403 & $\mathrm{x}$ & $0.0029 \pm 0.0048$ & $0.0004 \pm 0.0051$ \\
194.8 & $\mathrm{y}$ & $-0.0017 \pm 0.0045$ & $-0.0053 \pm 0.0048$ \\
176.2 & $\mathrm{z}$ & $0.0032 \pm 0.0053$ & $0.0014 \pm 0.0055$ \\
& $\mu_{\Xi-}$ & $-0.624 \pm 0.014$ & $-0.604 \pm 0.013$ \\
\hline 443 & $\mathrm{x}$ & $0.0114 \pm 0.0039$ & $0.0072 \pm 0.0041$ \\
267.5 & $\mathrm{y}$ & $0.0090 \pm 0.0039$ & $0.0020 \pm 0.0041$ \\
241.5 & $\mathrm{z}$ & $0.0039 \pm 0.0046$ & $-0.0009 \pm 0.0048$ \\
& $\mu_{\Xi-}$ & $-0.613 \pm 0.008$ & $-0.623 \pm 0.008$ \\
\hline Avg.= 393 & $\mathrm{x}$ & $0.0049 \pm 0.0020$ & $0.0033 \pm 0.0021$ \\
949.8 & $\mathrm{y}$ & $0.0046 \pm 0.0021$ & $0.0021 \pm 0.0021$ \\
872.0 & $\mathrm{z}$ & $-0.0012 \pm 0.0024$ & $-0.0031 \pm 0.0025$ \\
& $\mu_{\Xi-}$ & $-0.627 \pm 0.006$ & $-0.630 \pm 0.006$ \\
\hline & & \multicolumn{2}{|c}{}
\end{tabular}

Table 5.15: The measured $\Xi^{-}$polarizations the neutral production sample with and without the kinematic angle criteria $\int \mathrm{Bdl}=-24.36 \pm 0.24 \mathrm{~T} \mathrm{~m}$.

angle criteria at the $1 \sigma$ error. Since the statistical significance of these samples are at least a factor of 10 higher than the $\Omega^{-}$samples there is strong evidence that the kinematic angle cuts have no significant effect on the $\Omega^{-}$polarization results.

The $\Xi^{-}$also allows us to check the analysis by direct comparision of a $\Xi^{-}$magnetic moment result. This analysis used four spin transfer production $\Xi^{-}$samples of roughly equal size. Each of these samples was approximately 2.5 times as large as the final $\Omega^{-}$ sample and was analyzed using the same reconstruction and magnetic moment analysis as was used to analyze the $\Omega^{-}$result. Analysis of these samples allowed us to check the analysis in samples of higher statistical significance than the $\Omega^{-}$sample. Table 5.17 shows the momentum average polarization and magnetic moment results for the four spin transfer $\Xi^{-}$samples, as well as, the average results for the total sample. 


\begin{tabular}{c|c|c|c} 
Mom. $(\mathrm{GeV} / \mathrm{c})$ & View & $\cos \theta_{K}$ vs. $\Phi$ & no $\cos \theta_{K}$ vs. $\Phi$ \\
$10^{3}$ Events & & $\alpha_{\Lambda} \mathrm{P}_{\Lambda}$ & $\alpha_{\Lambda} \mathrm{P}_{\Lambda}$ \\
\hline 352 & $\mathrm{x}$ & $-0.0424 \pm 0.0057$ & $-0.0383 \pm 0.0059$ \\
103.2 & $\mathrm{y}$ & $-0.0109 \pm 0.0064$ & $-0.0169 \pm 0.0066$ \\
96.8 & $\mathrm{z}$ & $0.0188 \pm 0.0070$ & $0.0221 \pm 0.0072$ \\
\hline 380 & $\mathrm{x}$ & $-0.0552 \pm 0.0049$ & $-0.0586 \pm 0.0052$ \\
141.2 & $\mathrm{y}$ & $0.0048 \pm 0.0053$ & $0.0040 \pm 0.0056$ \\
130.4 & $\mathrm{z}$ & $0.0414 \pm 0.0058$ & $0.0427 \pm 0.0060$ \\
\hline 403 & $\mathrm{x}$ & $-0.0445 \pm 0.0064$ & $-0.0514 \pm 0.0067$ \\
104.5 & $\mathrm{y}$ & $-0.0028 \pm 0.0062$ & $-0.0009 \pm 0.0065$ \\
95.1 & $\mathrm{z}$ & $0.0480 \pm 0.0069$ & $0.0409 \pm 0.0073$ \\
\hline 443 & $\mathrm{x}$ & $-0.0773 \pm 0.0057$ & $-0.0728 \pm 0.0060$ \\
128.7 & $\mathrm{y}$ & $-0.0042 \pm 0.0058$ & $-0.0033 \pm 0.0062$ \\
107.6 & $\mathrm{z}$ & $0.0620 \pm 0.0066$ & $0.0691 \pm 0.0070$ \\
\hline Avg. $=393$ & $\mathrm{x}$ & $-0.0571 \pm 0.0028$ & $-0.0569 \pm 0.0030$ \\
460.8 & $\mathrm{y}$ & $-0.0015 \pm 0.0030$ & $-0.0030 \pm 0.0031$ \\
430.1 & $\mathrm{z}$ & $0.0413 \pm 0.0033$ & $0.0428 \pm 0.0034$ \\
\hline
\end{tabular}

Table 5.16: The measured $\Xi^{-}$polarizations the spin transfer production sample with and without the kinematic angle criteria $\int \mathrm{Bdl}=-24.36 \pm 0.24 \mathrm{~T} \cdot \mathrm{m}$.

\begin{tabular}{c|c|c|c}
$\begin{array}{c}\text { Sample } \\
10^{3} \text { Events }\end{array}$ & View & $\begin{array}{c}\text { Polarization } \\
\alpha_{\Lambda} \mathrm{P}_{\Lambda}\end{array}$ & $\begin{array}{c}\mu_{\Xi^{-}} \\
\mu_{N}\end{array}$ \\
\hline I & $\mathrm{x}$ & $-0.0567 \pm 0.0026$ & \\
413.0 & $\mathrm{y}$ & $0.0024 \pm 0.0029$ & $-0.6552 \pm 0.0062$ \\
& $\mathrm{z}$ & $0.0316 \pm 0.0049$ & \\
\hline II & $\mathrm{x}$ & $-0.0517 \pm 0.0030$ & \\
327.8 & $\mathrm{y}$ & $0.0025 \pm 0.0032$ & $-0.6364 \pm 0.0073$ \\
& $\mathrm{z}$ & $0.0334 \pm 0.0076$ & \\
\hline III & $\mathrm{x}$ & $-0.0643 \pm 0.0028$ & \\
365.7 & $\mathrm{y}$ & $0.0022 \pm 0.0030$ & $-0.66529 \pm 0.0061$ \\
& $\mathrm{z}$ & $0.0306 \pm 0.0035$ & \\
\hline IV & $\mathrm{x}$ & $-0.0683 \pm 0.0031$ & \\
413.0 & $\mathrm{y}$ & $0.0040 \pm 0.0034$ & $-0.6545 \pm 0.0063$ \\
& $\mathrm{z}$ & $0.0418 \pm 0.0080$ & \\
\hline Total & $\mathrm{x}$ & $-0.0600 \pm 0.0014$ & \\
1519.5 & $\mathrm{y}$ & $0.0033 \pm 0.0016$ & $-0.6478 \pm 0.0032$ \\
& $\mathrm{z}$ & $0.0316 \pm 0.0017$ & \\
\hline
\end{tabular}

Table 5.17: The momentum averaged measured $\Xi^{-}$polarizations and magnetic moments for four spin transfer $\Xi^{-}$samples at $\int \mathrm{Bd} l=-24.36$. 
The previous measurements for this production method give a magnetic moment result of:

$$
\mu_{\Xi-}=-0.6505 \pm 0.0025 \mu_{N}
$$

This is in good agreement with the results for the four individual cascade samples and the total spin production cascade sample given by:

$$
\mu_{\Xi^{-}}=-0.6478 \pm 0.0032 \mu_{N} .
$$

The level of agreement of the systematic studies and the results of the consistency checks demonstrate that the systematic error present in $\mu_{\Omega^{-}}$analysis is well below the level of the statistical error so we need only quote a statistical error for this measurement. 


\section{Chapter 6}

\section{Implications}

In Chapter 1 many methods and models for predicting the magnetic moments of baryons were discussed. In Table 1.6 listed theoretical predictions for $\mu_{\Omega^{-}}$and the previous experimental measurement. Table 6.1 includes the final result of this experiment in the table for comparison both to the theoretical predictions and the previous measurement.

\begin{tabular}{l|c|c} 
Model & $\mu_{\Omega^{-}}$ & Reference \\
\hline SQM & -1.83 & Table 1.2 \\
SQM mass correction & -1.3 & {$[12]$} \\
SQM mass correction & $-1.52-1.48$ & {$[14]$} \\
SQM charge correction & -2.33 & {$[15]$} \\
Relativistic Bag Model & $-1.95-2.52$ & {$[32]$} \\
LGT & $-1.7 \pm .6$ & {$[34]$} \\
LGT & $-1.73 \pm .22$ & {$[35]$} \\
Measured value Fermilab E756 & $-1.94 \pm 0.17 \pm 0.14$ & {$[7]$} \\
Measured value this thesis & $-2.024 \pm 0.056$ & -
\end{tabular}

Table 6.1: Theoretical predictions for the $\Omega^{-}$magnetic moment. The previously measured value is included for comparison.

The result presented in this dissertation is in good agreement with the previous measurement. All but one of the theoretical predictions are in disagreement with our measurement to $3 \sigma$. Only the the relativistic bag model [32] which is within $1 \sigma$ is in agreement with this measurement. However the choice of parameters for the bag model 
which gives a prediction close to the measured value does not give good predictions for all the other baryons. Figure 6.1 shows the difference between the predictions of this bag model and the measured values for the baryons in nuclear magnetons with the experimental error bars.

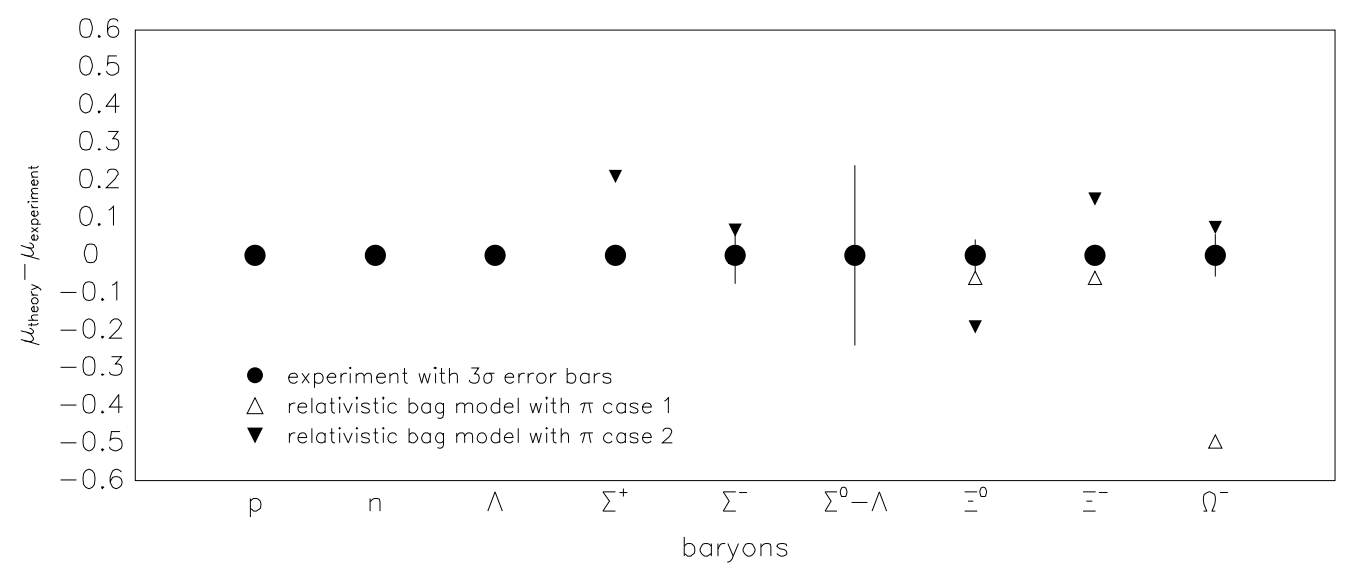

Figure 6.1: Comparison of experimental measurements of baryon magnetic moments with the theory which provides the best $\mu_{\Omega^{-}}$value. The y-axis is the difference between theory and experiment in units of nuclear magnetons. The two cases include two conditions on the parameters used in this model.

The above mentioned model manages to fit both the $\Omega^{-}$and $\Sigma^{-}$or the $\Xi^{-}$and $\Xi^{0}$. However, this model makes use of two parameters and also uses the proton, neutron and, $\Lambda$ moments as inputs. So with two free parameters this model can fit two baryons other than those used as inputs. The predictions for other baryons are several standard deviations from the measured values. We can conclude that this model does not reproduce the experimental data.

The disagreement of theoretical predictions and experimental measurement is an indication that the baryons are very complex systems. Even the $\Omega^{-}$with its simple valence quark configuration cannot be predicted reliably by theory. 
Further eveidence of the complexity of the baryonic system is the recent measurements of the CERN SMC collaboration and SLAC experiments 142 and 143. The deep inelastic scattering results of muons and protons and neutrons measures the fraction of the baryon spin carried by the valence quarks. results presented recently conclude that the measurements of these groups are in agreement and that only approximately $30 \%$ of the total spin of the neutron and proton is carried by the valence quarks [48, 49].

One can approach these findings from two directions. One is to ask "How can we hope to predict baryon magnetic moments without considering quark anti-quark pairs and gluons in the system?". The other appraoch is to ask "How is it that the naive quark model predicts the magnetic moments of the baryons as well as it does given that it completely ignores this effect?".

The baryonic system is the most complex system under consideration by high energy physicists. The proton and neutron are stable and easily investigated. Yet the structure of even these particle is not well understood. Hopefully the measurement of the magnetic moments of the baryons and in particular the simple $\Omega^{-}$system when combined with deep inelastic scattering measurements will revive interest in baryon structure calculations among theorists. 


\section{References}

[1] Cohen et al., Rev. Mod. Phys. 591121 (1986).

[2] L. Schachinger et al., Phys Rev Lett. 411348 (1978).

[3] A Morelos et al., Phys Rev Lett. 713417 (1993).

[4] P. C. Peterson et al., Phys Rev Lett. 57949 (1986).

[5] G. Zapalac et al., Phys Rev Lett. 571526 (1986).

[6] P. T. Cox et al., Phys Rev Lett. 46877 (1981).

[7] J. Duryea et al., Phys. Lett. 68, 768 (1992).

[8] H. T. Diehl et al., Phys. Lett. 67, 804 (1991).

[9] L. Brekke and J. Rosner, Comm. Nucl. Part. Phys. 18, 83 (1988).

[10] H. Georgi and A. Manohar, Phys. Lett., 132B, 183 (1983).

[11] R. B. Teese and R. Settles, Phys. Lett. 87B, 111 (1979).

[12] T. Das and S. Misra, Phys. Lett. 96B, 165 (1980).

[13] I. S. Sogami and N. O. Oh'yamaguchi, Phys. Rev. Lett. 54, 2295 (1985).

[14] Y. Tomozawa, Phys. Rev. D 25, 795 (1982).

[15] R. Verma and M. Khanna, Phys. Lett. 183B, 207 (1987). 
[16] N. Isgur and G. Karl, Phys. Rev. D 21, 3175 (1980).

[17] Z. Dziembowski et al., Phys. Lett. 200B, 539 (1988).

[18] R. B. Teese, Phys. Rev. D 24, 1413 (1980).

[19] S. K. Gupta and S. B. Khadkikar, Phys. Rev. D 36, 307 (1987).

[20] R. Verma, Phys. Rev. D. 22, 1156 (1980).

[21] G. E. Brown et al., Phys. Lett. 97B, 423 (1980).

[22] S. Coleman and S. L. Glashow, Phys. Lett. 6, 423 (1961).

[23] W. Thirring, Acta Physica Austrica (1966).

[24] H. J. Lipkin, Phys. Rev. Lett. 41, 1629 (1978).

[25] B. Ioffe and A. Smilga, Phys. Lett. 133B, 463 (1983).

[26] C. B. Chiu et al., Phys. Rev. D 36, 1553 (1987). 1839 (1985).

[27] H. J. Lipkin, Phys. Rev. D. 24, 1437 (1981).

[28] A. Chodos et al., Phys. Rev. D 102599 (1974)

[29] T. DeGrandet al., Phys. Rev. D 122060 (1975)

[30] Z. Dziembowski and L. Mankiewicz, Phys. Rev. Lett. 55,

[31] S. Theberge and A. Thomas, Nucl. Phys. A393, 252 (1983).

[32] M. I. Krivoruchenko, Sov. J. Nucl. Phys. 45, 109 (1987).

[33] J. Cohen and H. J. Weber, Phys. Lett. 165B, 229 (1985).

[34] C. Bernard et al., Phys. Rev. Lett. 49, 1076 (1982).

[35] D.W. Lienweber Phys. Rev. D 45, 252 (1992) 
[36] K. Hikasa et al. Phys. Rev. D 45 Part II VIII.111 (1992).

[37] H. J. Lipkin Nucl. Phys. B241 477 (1984).

[38] V. Bargman et al. Phys. Rev. Lett. 2, 435 (1959).

[39] J. Duryea Ph. D. Thesis, University of Minnesota (unpublished) (1991).

[40] K.B. Luk et al. Phys. Rev. Lett. 70, 900 (1993).

[41] G. Bunce et al., Phys. Rev. Lett. 36, 1113 (1976).

[42] B. Lundberg et al., Phys. Rev. D 40, 3557 (1989).

[43] K. Heller et al., Phys. Rev. Lett. 51, 2025 (1983).

[44] H. Haggerty et al., NIM 115, 157 (1974)

[45] K.B. Luk, Ph.D. thesis, Rutgers University (unpublished)(1983).

[46] J. Kim et al., Phys. Rev. D 46, 1060 (1992).

[47] Gerald Guglielmo Ph.D. thesis University of Minnesota (unpublished)(1994).

[48] D. B. Day et. al. to be published in Proceedings of the 1994 International Spin Conference.

[49] R. Windmolders et. al. to be published in Proceedings of the 1994 International Spin Conference. 